Regularity at infinity and global fibrations of real algebraic maps

\author{
Luis Renato Gonçalves Dias
}


SERVIÇO DE PÓS-GRADUAÇÃO DO ICMC-USP

Data de Depósito:

Assinatura:

\title{
Regularity at infinity and global fibrations of real algebraic maps
}

\author{
Luis Renato Gonçalves Dias \\ Advisors: Professor Maria Aparecida Soares Ruas \\ Professor Mihai Marius Tibar
}

Doctoral dissertation submitted to the Instituto de Ciências Matemáticas e de Computação - ICMC-USP, in partial fulfillment of the requirements for the degree of the Doctorate Program in Mathematics. EXAMINATION BOARD PRESENTATION COPY.

USP - São Carlos

November 2012 
Ficha catalográfica preparada pela Seção de Tratamento da Informação da Biblioteca Prof. Achille Bassi - ICMC/USP

Dias, Luis Renato Gonçalves
Regularity at infinity and global fibrations of real
algebraic maps / Luis Renato Gonçalves Dias ;
orientadores Maria Aparecida Soares Ruas e Mihai Tibar.
-- São Carlos, 2012.
83 p.
Tese (Doutorado Duplo - Programa de Pós-Graduação em
Matemática and Doctorat en Mathématique) - Instituto de
Ciências Matemáticas e de Computação, Universidade de São
Paulo; Université Lille I, 2012.
1. Bifurcation values. 2. Atypical values. 3.
Regularity conditions at infinity. 4. t-regularity. 5.
rho-regularity. 6. Asymptotic critical values. 7. Morse-
Sard type theorem. 8. Integral closure. 9. Newton non-
degeneracy. I. Ruas, Maria Aparecida Soares, orient. II.
Tibar, Mihai, orient. III. Título.


SERVIÇO DE PÓS-GRADUAÇÃO DO ICMC-USP

Data de Depósito:

Assinatura:

\title{
Regularidade no infinito e fibrações globais de aplicações algébricas reais
}

\author{
Luis Renato Gonçalves Dias \\ Orientadores: Professora Maria Aparecida Soares Ruas \\ Professor Mihai Marius Tibar
}

Tese apresentada ao Instituto de Ciências Matemáticas e de Computação - ICMC-USP, como parte dos requisitos para obtenção do título de Doutor em Ciências - Matemática. EXEMPLAR DE DEFESA. 
Ficha catalográfica preparada pela Seção de Tratamento da Informação da Biblioteca Prof. Achille Bassi - ICMC/USP

Dias, Luis Renato Gonçalves
Regularidade no infinito e fibrações globais de
aplicações algébricas reais / Luis Renato Gonçalves
Dias ; orientadores Maria Aparecida Soares Ruas e Mihai
Tibar. -- São Carlos, 2012 .
83 p.
Tese (Doutorado Duplo - Programa de Pós-Graduação em
Matemática e Doctorat en Mathématique) - Instituto de
Ciências Matemáticas e de Computação, Universidade de São
Paulo; Université Lille I, 2012 .
1. Valores de bifurcação. 2. Valores atípicos. 3.
Condições de regularidade no infinito. 4. t-regularidade.
5. rho-regularidade. 6. Valores críticos assintóticos. 7.
Teoremas tipo Morse-Sard. 8. Fecho integral. 9. Newton
degeneracidade. I. Ruas, Maria Aparecida Soares, orient.
II. Tibar, Mihai, orient. III. Título.



A minha querida esposa fosiani:

Hos meus queridos pais, Fernando e fussara. 



\section{Acknowledgements}

I would like to express my thanks to all those who contributed in many ways to the success of this work and made it an unforgettable experience for me.

I cannot find words to express my gratitude to my advisors, Professor Maria Aparecida Soares Ruas and Professor Mihai Marius Tibăr, who offered invaluable assistance, support and guidance, who helped me patiently in so many ways and inspired me. To work with you has been a great pleasure for me. Muito obrigado mesmo!

I wish to express my thanks to Ying Chen, Professor Nilva R. Ribeiro and Professor Raimundo N. A. dos Santos for numerous useful discussions.

I would like to thank Professor Carles Bivià-Ausina for all the assistance and generosity during my short stay at Valencia, and for some useful discussions. Thanks a lot!

I wish to thank to Professor Terence Gaffney and Professor Zbigniew Jelonek for accepting to be the referees of this thesis.

I take this opportunity to sincerely acknowledge all members of ICMC-USP (São Carlos-Brazil) for their support and for providing a pleasant and productive working atmosphere. I also thank all members of Laboratoire Paul Painlevé (Lille-France) for making the visiting period (from 10/2010 to 10/2011) pleasant and productive.

I thank the financial support provided by Brazilian grants FAPESP (Proc. 2008/10563-4) and CAPES (Proc. 2929/10-04).

My wife Josiani and I thank Tibăr's family for all the generosity, kindness and assistance in all moments of our stay at Lille.

I wish to express my love and gratitude to my beloved families; for their understanding, encouragement and endless love throughout my studies, Josiani; Fernando and Jussara; Eduardo (Dú), Cibeli (Beli), Paulo (Branco), Karine (Ká) and Bianca (Bi); Ligia, Cristiano, Angela and Gustavo; Mario and Norma. Vocês todos são demais! 



\section{Abstract}

Let $f: \mathbb{K}^{n} \rightarrow \mathbb{K}^{p}$ be a $C^{2}$ semi-algebraic mapping for $\mathbb{K}=\mathbb{R}$ and a polynomial mapping for $\mathbb{K}=\mathbb{C}$. It is well-known that $f$ is a locally trivial topological fibration over the complement of the bifurcation set $B(f)$, also called atypical set.

In this work, we consider the notion of t-regularity and $\rho_{E}$-regularity to study the bifurcation set of semi-algebraic mappings $f: \mathbb{R}^{n} \rightarrow \mathbb{R}^{p}$ and polynomial mappings $f: \mathbb{C}^{n} \rightarrow \mathbb{C}^{p}$.

We show that $t$-regularity is equivalent to regularity conditions at infinity which have been used by Rabier (1997), Gaffney (1999), Kurdyka, Orro and Simon (2000) and Jelonek (2003) in order to control the asymptotic behaviour of mappings. In addition, we prove that $t$-regularity implies $\rho_{E}$-regularity.

The $\rho_{E^{-}}$-regularity enables one to define the set of asymptotic non $\rho_{E^{-}}$-regular values $S(f) \subset \mathbb{K}^{p}$, and the set $A_{\rho_{E}}:=f(\operatorname{Sing} f) \cup S(f)$. For $C^{2}$ semi-algebraic mappings $f: \mathbb{R}^{n} \rightarrow \mathbb{R}^{p}$ and polynomial mappings $f: \mathbb{C}^{n} \rightarrow \mathbb{C}^{p}$, based on a partial Thom stratification at infinity, we prove that $S(f)$ and $A_{\rho_{E}}$ are closed real semi-algebraic sets of dimension at most $p-1$ (real dimension at most $2 p-2$, for $f: \mathbb{C}^{n} \rightarrow \mathbb{C}^{p}$ ). Moreover, based on a new fibration theorem "at infinity", i.e. holding in the complement of a sufficiently large ball, we obtain $B(f) \subset A_{\rho_{E}}$.

We study two special classes of polynomial mappings $f: \mathbb{R}^{n} \rightarrow \mathbb{R}^{p}$, the class of fair polynomial mappings and the class of Newton non-degenerate polynomial mappings. For fair polynomial mappings, we give an interpretation of $t$-regularity in terms of integral closure of modules, which is a real counterpart of Gaffney's result (1999). For non-degenerate polynomial mappings, we obtain an approximation for $B(f)$ through a set which depends on the Newton polyhedron of $f$ (results like this have been obtained by Némethi and Zaharia (1990) for polynomial functions $f: \mathbb{C}^{n} \rightarrow \mathbb{C}$ and recently for mixed polynomial functions by Chen and Tibăr (2012)).

To finish, we discuss some simple consequences of our work: the equivalence $t$ regularity $\Leftrightarrow$ Rabier (equivalently Gaffney, Kuo-KOS, Jelonek) condition for mappings $f: X \rightarrow \mathbb{K}^{p}$, where $X \subset \mathbb{K}^{n}$ is a smooth affine variety; the problem of bijectivity of semi-algebraic mappings; and a formula to compute the Euler characteristic of regular fibres of polynomial mappings $f: \mathbb{R}^{n} \rightarrow \mathbb{R}^{n-1}$.

The above results are also extensions of some results obtained, for polynomial functions $f: \mathbb{K}^{n} \rightarrow \mathbb{K}$, by Némethi and Zaharia (1990), Siersma and Tibăr (1995), Păunescu and Zaharia (1997), Parusiński (1995) and Tibăr (1998).

Title: Regularity at infinity and global fibrations of real algebraic maps.

Key words: bifurcation values, atypical values, regularity conditions at infinity, $t$ regularity, $\rho_{E}$-regularity, asymptotic critical values, Morse-Sard type theorem, integral closure, Newton non-degeneracy. 



\section{Resumo}

Considere $f: \mathbb{K}^{n} \rightarrow \mathbb{K}^{p}$ uma aplicação semi-algébrica de classe $C^{2}$ para $\mathbb{K}=\mathbb{R}$ e uma aplicação polinomial para $\mathbb{K}=\mathbb{C}$. Por resultados clássicos, sabe-se que $f$ é uma fibração topologicamente trivial sobre o complementar dos valores de bifurcação $B(f)$, também chamado de valores atípicos.

Neste trabalho, consideramos a $t$-regularidade e a $\rho_{E}$-regularidade no estudo dos valores de bifurcação de aplicações semi-algébricas $f: \mathbb{R}^{n} \rightarrow \mathbb{R}^{p}$ de classe $C^{2}$ e aplicações polinomiais $f: \mathbb{C}^{n} \rightarrow \mathbb{C}^{p}$.

Mostramos que $t$-regularidade é equivalente às condições de regularidade no infinito usadas por Rabier (1997), Gaffney (1999), Kurdyka, Orro e Simon (2000) e Jelonek (2003) no controle do comportamento assintótico de aplicações. Também mostramos que $t$-regularidade implica $\rho_{E}$-regularidade.

Através da $\rho_{E^{-}}$regularidade, definimos o conjunto dos valores assintóticos não $\rho_{E^{-}}$ regulares $S(f) \subset \mathbb{K}^{p}$, e o conjunto $A_{\rho_{E}}:=f(\operatorname{Sing} f) \cup S(f)$. Para aplicações semialgébricas $f: \mathbb{R}^{n} \rightarrow \mathbb{R}^{p}$ de classe $C^{2}$ e aplicações polinomiais $f: \mathbb{C}^{n} \rightarrow \mathbb{C}^{p}$, baseados na existência de uma estratificação parcial de Thom no infinito, provamos que $S(f)$ e $A_{\rho_{E}}$ são conjuntos semi-algébricos reais de dimensão no máximo $p-1$ (dimensão real no máximo $2 p-2$, para $f: \mathbb{C}^{n} \rightarrow \mathbb{C}^{p}$ ). Além disso, baseados em um novo teorema de fibração "no infinito", ou seja na existência de fibração no complementar de uma bola de raio suficientemente grande, obtemos que o conjunto de bifurcação $B(f)$ está contido no conjunto $A_{\rho_{E}}$.

Estudamos também duas classes de aplicações polinomiais $f: \mathbb{R}^{n} \rightarrow \mathbb{R}^{p}$, a classe de aplicações polinomiais "fair" e a classe de aplicações Newton não degeneradas. Para aplicações polinomiais fair, obtemos uma interpretação da $t$-regularidade em termos da teoria de fecho integral de módulos, estendendo para o caso real os resultados de Gaffney (1999). Para aplicações não degeneradas, obtemos uma aproximação de $B(f)$ através de um conjunto que depende do poliedro de Newton de $f$ (resultados deste tipo foram obtidos por Némethi e Zaharia (1990) para funções polinomiais $f: \mathbb{C}^{n} \rightarrow \mathbb{C}$ e recentemente para funções polinomiais mistas por Chen e Tibăr (2012)).

No final, discutimos algumas consequências simples do nosso trabalho: a equivalência $t$-regularidade $\Leftrightarrow$ condição de Rabier (equivalentemente Gaffney, Kuo-KOS, Jelonek) para aplicações $f: X \rightarrow \mathbb{K}^{p}$, onde $X \subset \mathbb{K}^{n}$ é uma variedade suave afim; o problema de bijetividade de aplicações semi-algébricas; e uma fórmula para o cálculo da característica de Euler de fibras regulares de aplicações polinomiais $f: \mathbb{R}^{n} \rightarrow \mathbb{R}^{n-1}$.

Os resultados acima também são extensões de alguns resultados obtidos para funções polinomiais $f: \mathbb{K}^{n} \rightarrow \mathbb{K}$, por Némethi e Zaharia (1990), Siersma e Tibăr (1995), Păunescu e Zaharia (1997), Parusiński (1995) e Tibăr (1998).

Título: Regularidade no infinito e fibraçôes globais de aplicaçôes algébricas reais.

Palavras chaves: valores de bifurcação, valores atípicos, condições de regularidade no infinito, $t$-regularidade, $\rho_{E}$-regularidade, valores críticos assintóticos, teoremas tipo Morse-Sard, fecho integral, Newton não degeneracidade. 



\section{Résumé}

Soit $f: \mathbb{K}^{n} \rightarrow \mathbb{K}^{p}$ une application semi-algébrique de classe $C^{2}$ pour $\mathbb{K}=\mathbb{R}$, ou une application polynomiale pour $\mathbb{K}=\mathbb{C}$. Il est bien connu que $f$ est une fibration localement triviale sur le complémentaire des valeurs de bifurcation $B(f)$ (aussi appelés valeurs atypiques).

Dans ce travail nous considérons la $t$-régularité et la $\rho_{E}$-régularité dans l'étude des valeurs de bifurcation des applications semi-algébriques $f: \mathbb{R}^{n} \rightarrow \mathbb{R}^{p}$ de classe $C^{2}$ et des applications polynomiales $f: \mathbb{C}^{n} \rightarrow \mathbb{C}^{p}$.

Nous démontrons que $t$-régularité est équivalent aux conditions de régularité à l'infini de Rabier (1997), Gaffney (1999), Kurdyka, Orro et Simon (2000) et Jelonek (2003). On démontre que $t$-régularité implique $\rho_{E}$-régularité.

Avec la $\rho_{E^{-}}$régularité, on définit l'ensemble des valeurs asymptotique non $\rho_{E^{-}}$ régulières $S(f) \subset \mathbb{K}^{p}$, et l'ensemble $A_{\rho_{E}}:=f(\operatorname{Sing} f) \cup S(f)$. Pour les applications semi-algébriques $f: \mathbb{R}^{n} \rightarrow \mathbb{R}^{p}$ de classe $C^{2}$ et applications polynomiales $f: \mathbb{C}^{n} \rightarrow \mathbb{C}^{p}$, en s'appuyant sur l'existence des stratifications partielles de Thom à l'infini, on prouve que $S(f)$ et $A_{\rho_{E}}$ sont des ensembles semi-algébriques réels de dimension $\leq p-1$ et de dimension réelle $\leq 2 p-2$, pour $f: \mathbb{C}^{n} \rightarrow \mathbb{C}^{p}$. En s'appuyant sur un théorème de fibration "à l'infini", on démontre l'inclusion $B(f) \subset A_{\rho_{E}}$.

Nous étudions aussi deux classes d'applications polynomiales $f: \mathbb{R}^{n} \rightarrow \mathbb{R}^{p}$, les applications polynomiales "fair" et les applications Newton non dégénérées. Pour les applications fair, on obtient une interprétation de la t-régularité en termes de la théorie de la clôture intégrale des modules. Ce type de résultat apparaît dans un article de Gaffney (1999) pour les applications polynomiales $f: \mathbb{C}^{n} \rightarrow \mathbb{C}^{p}$. Pour les applications Newton non dégénérées, nous obtenons une approximation de $B(f)$, ce qui étende le résultat de Némethi et Zaharia (1990) pour les fonctions polynomiales $f: \mathbb{C}^{n} \rightarrow \mathbb{C}$ et celui de Chen et Tibăr (2012) pour les fonctions polynomiales mixtes.

Dans la dernière partie, on discute quelques conséquences: 1).l'équivalence $t$ régularité $\Leftrightarrow$ les conditions de Rabier, Gaffney, Kuo-KOS, Jelonek pour les applications $f: X \rightarrow \mathbb{K}^{p}$, où $X \subset \mathbb{K}^{n}$ est une variété lisse; 2).le problème de bijectivité des applications semi-algébriques; et 3).une formule pour calculer la caractéristique d'Euler des fibres régulières des applications polynomiales $f: \mathbb{R}^{n} \rightarrow \mathbb{R}^{n-1}$.

Les résultats présentés brièvement ci-dessus généralisent aussi certains résultats de Némethi et Zaharia (1990), Siersma et Tibăr (1995), Păunescu et Zaharia (1997), Parusiński (1995) et Tibăr (1998).

Titre: Régularité à l'infini et fibrations globales des applications algébriques réelles.

Mots clés: valeurs de bifurcation, valeurs atypiques, condition de régularité à l'infini, $t$-régularité, $\rho_{E}$-régularité, valeurs critiques asymptotiques, théorèmes de type MorseSard, clôture intégrale, polyèdre de Newton à l'infini. 



\section{Contents}

$\begin{array}{ll}\text { Introduction } & 1\end{array}$

1 Preliminaries $\quad 7$

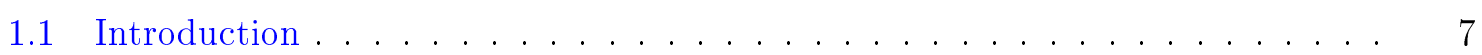

1.2 Basic Definitions . . . . . . . . . . . . . . . . . . 8

1.3 Rabier, Gaffney, Kuo, Jelonek functions . . . . . . . . . . . . . . . . 10

1.4 Relative Functions . . . . . . . . . . . . . . . . . . . . . . . 14

$\begin{array}{lll}2 & \text { Regularity conditions at infinity } & 17\end{array}$

2.1 Introduction . . . . . . . . . . . . . . . . . . . . . . . 18

2.2 Polynomial functions . . . . . . . . . . . . . . . . . . . . 20

2.3 Affine regularity conditions at infinity for mappings . . . . . . . . . . . 24

2.3.1 Fibration Theorem . . . . . . . . . . . ..... 25

2.3 .2 Structure Theorem . . . . . . . . . . . . . . . . . 26

2.3.3 Asymptotic Morse-Sard type theorem . . . . . . . . . . . . 26

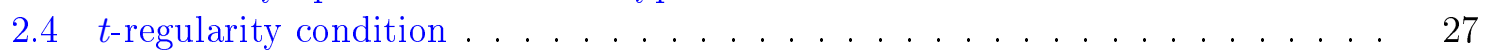

2.4.1 Localized version of some regularity conditions . . . . . . . . . 28

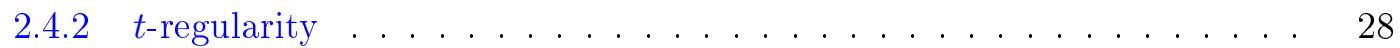

2.4.3 Regularity conditions and the $t$-regularity . . . . . . . . . . . 30

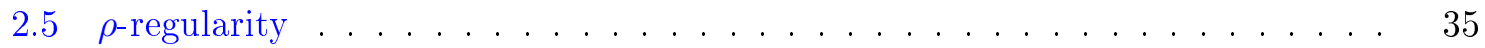

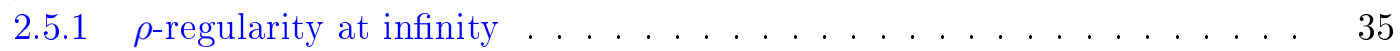

2.5.2 t-regularity and $\rho_{E}$-regularity $\ldots \ldots \ldots \ldots$

3 Asymptotic theorems for $\rho_{E}$-regularity $\quad 39$

3.1 Morse-Sard type theorem for $\rho_{E}$-regular values $\ldots \ldots \ldots \ldots$. . . . . . . 40

3.1.1 Partial Thom stratification at infinity . . . . . . . . . . . . . 41

3.1.2 Proof of Theorem 3.1.1 . . . . . . . . . . . . . . . . . 42

3.1.3 Complex Case . . . . . . . . . . . . . . . . . . 45

3.2 Diagram . . . . . . . . . . . . . . . . . . . 46

3.3 Example . . . . . . . . . . . . . . . . . . . . 47

4 Polynomial mappings $\quad 49$

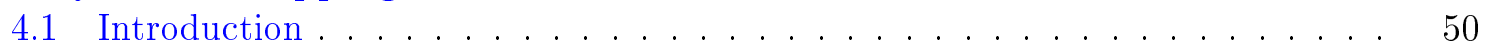

4.2 Integral closure of modules and $t$-regularity $\ldots \ldots \ldots \ldots \ldots$

4.2 .1 Real integral closure of modules . . . . . . . . . . . . . . 51

4.2 .2 t-regularity and polynomial mappings . . . . . . . . . . . 53

4.3 Newton polyhedron and bifurcation values . . . . . . . . . . . . 56

4.3.1 Newton polyhedron . . . . . . . . . . . . . . . . 57

4.3.2 Newton polyhedron and polynomial mappings . . . . . . . . . . 59

4.3 .3 Non-degeneracy conditions at infinity . . . . . . . . . . . . . . . 62

$\begin{array}{llr}5 & \text { Some consequences } & 69\end{array}$

5.1 Relative Case . . . . . . . . . . . . . . . . . . 70

5.2 Bijectivity of semi-algebraic mappings . . . . . . . . . . . . 71

5.3 Euler characteristic . . . . . . . . . . . . . . . . . . . . . . 74 


\section{Introduction}

The main object of this thesis is the following:

Definition 1 (Bifurcation values (or Atypical values)). Let $f: \mathbb{K}^{n} \rightarrow \mathbb{K}^{p}, n \geq p$, be a $C^{1}$ semi-algebraic mapping for $\mathbb{K}=\mathbb{R}$ and a complex polynomial mapping for $\mathbb{K}=\mathbb{C}$. We say that $f$ is topologically trivial at $t_{0} \in \mathbb{K}^{p}$ if there exists a neighbourhood $U$ of $t_{0}$ in $\mathbb{K}^{p}$ such that the restriction $f_{\mid}: f^{-1}(U) \rightarrow U$ is a topologically trivial fibration (i.e. there exists a homeomorphism $h: f^{-1}\left(t_{0}\right) \times U \rightarrow f^{-1}(U)$ such that $f \circ h=p r_{2}$, where $p r_{2}: f^{-1}\left(t_{0}\right) \times U \rightarrow U$ denotes the second projection). If one point $t \in \mathbb{K}^{p}$ does not satisfy this property, then we say that $t$ is a bifurcation value $e^{1}$ of $f$. We shall denote by $B(f)$ the set of bifurcation values of $f$.

REMARK 1. Let $f: \mathbb{K}^{n} \rightarrow \mathbb{K}^{p}$ be a $C^{1}$ semi-algebraic mapping for $\mathbb{K}=\mathbb{R}$ and a complex polynomial mapping for $\mathbb{K}=\mathbb{C}$. Directly from the above definition, if $t_{0} \notin B(f)$ then there exists a neighbourhood $U$ of $t_{0}$ in $\mathbb{K}^{p}$ such that, for any $t \in U, f^{-1}(t)$ is homeomorphic to $f^{-1}\left(t_{0}\right)$. Other direct consequences on $B(f)$ are that the topological type of the fibres of $f$ depend on the connected components of $\mathbb{K}^{p} \backslash B(f)$ and that $B(f) \supset \overline{\operatorname{Im} f} \backslash \operatorname{Im} f$.

There are interesting connections between bifurcation values and many other topics such as problems of optimization of polynomial functions $f: \mathbb{R}^{n} \rightarrow \mathbb{R}$ (see e.g. Hà and Pham [23]), generalizations of Ehresmann's Theorem (see e.g. Rabier [48], Gaffney [18], Jelonek [24]), Jacobian Conjecture (see e.g. Lê and Weber [30], Siersma and Tibăr [49]), generalization of Morse theory (see e.g. Palais and Smale [41]), global Łojasiewicz exponents (see e.g. Păunescu and Zaharia [45], D'Acunto and Grandjean [9]), equisingularity and Milnor numbers (see e.g. Gaffney [18], Parusiński [42, 44], Siersma and Tibăr [49], Tibăr [55, 56]), Thom conjecture (see Kurdyka, Mostowski and Parusiński [28]), stratification theory (see e.g. Tibăr [54], Kurdyka, Orro and Simon [29]), etc...

The difficulty to describe the bifurcation values of $f: \mathbb{K}^{n} \rightarrow \mathbb{K}^{p}$ resides in the fact that, since $f: \mathbb{K}^{n} \rightarrow \mathbb{K}^{p}$ can be not proper, we cannot use the classical Ehresmann's Theorem [14] to verify if $f$ is topologically trivial at a regular point $t_{0}$. Indeed, the bifurcation values may come from the critical values but also from the asymptotic behaviour of the fibres, i.e. the set $B(f) \backslash(B(f) \cap f(\operatorname{Sing} f))$ may be not empty (see for instance Example 2.1.1 (page 18)).

A complete characterization of $B(f) \backslash(B(f) \cap f(\operatorname{Sing} f))$ is yet an open problem. In fact, a characterization for this set is available only in the case of polynomial functions $f: \mathbb{K}^{2} \rightarrow \mathbb{K}$, see Suzuki [50], Hà and Lê [22] for $\mathbb{K}=\mathbb{C}$ and Tibăr and Zaharia [57] for $\mathbb{K}=\mathbb{R}$ (see also $\S 2.2$ (page 20)).

\footnotetext{
${ }^{1}$ also called of atypical value in the literature.
} 
One has therefore imagined various ways to characterize the sets $B(f)$ and $B(f) \backslash(B(f) \cap$ $f(\operatorname{Sing} f))$, essentially through the use of regularity conditions at infinity.

For polynomial functions $f: \mathbb{K}^{n} \rightarrow \mathbb{K}$, starting with the work of Broughton [4] (see also Pham [46]), we can find many works in this direction. For $f: \mathbb{C}^{n} \rightarrow \mathbb{C}$, Broughton $[4,5]$ worked with a Palais-Smale type condition called tame, later extended by Némethi [38, 39] to quasitame and by Némethi and Zaharia [40] to M-tame (also called $\rho_{E}$-regularity), a Milnor type condition of transversality of $f$ to the Euclidean distance function $\rho_{E}$. Parusinski [42] used the Malgrange condition (which appeared in Pham [47, page 14] and it is a Łojasiewicz type condition at infinity) and versions of it. Siersma and Tibăr worked with the $t$-regularity (also called $t$-equisingularity) [49,53], which is a type of non-characteristic condition at infinity, see also Parusiński [42]. For $f: \mathbb{R}^{n} \rightarrow \mathbb{R}$, the $t$-regularity and the $\rho_{E}$-regularity were also considered by Tibăr [55]. One finds a detailed discussion of the relations between these conditions in Némethi and Zaharia [40], Durfee [13] for the complex setting and in Tibăr $[55,56]$ for the real and complex settings. See $§ 2.2$.

Let us turn to the case of semi-algebraic mappings $f: \mathbb{R}^{n} \rightarrow \mathbb{R}^{p}$ and polynomial mappings $f: \mathbb{C}^{n} \rightarrow \mathbb{C}^{p}$.

Rabier [48] considered a metric-type regularity condition, which we call here Rabier condition. From this condition, he defined the set of asymptotic critical values $K_{\infty}(f)$ and proved that $B(f) \subset\left(f(\operatorname{Sing} f) \cup K_{\infty}(f)\right)$. In fact, Rabier's results apply to $C^{2}$ maps $f: M \rightarrow N$, where $M, N$ are Finsler Manifolds.

In his study of polynomial mappings $f: \mathbb{C}^{n} \rightarrow \mathbb{C}^{p}$, Gaffney [18] defined the generalized Malgrange condition, which we shall call here Gaffney condition. Under additional hypothesis on $f$, Gaffney proved that his condition yields a set $A_{G_{\infty}}(f)$ of non-regular values at infinity so that $B(f) \subset\left(f(\operatorname{Sing} f) \cup A_{G_{\infty}}(f)\right)$. Then, he used the theory of integral closure of modules to relate this condition to a non-characteristic condition like in Parusiński [42].

Kurdyka, Orro and Simon [29] also considered Rabier condition. They obtained an equivalence $([29, \S 2.2])$ between Rabier condition and another condition which depends on the Kuo function (we call this last condition Kuo-KOS condition). Then, for $C^{2}$ semi-algebraic mappings $f: \mathbb{R}^{n} \rightarrow \mathbb{R}^{p}$ (respectively, for polynomial mappings $f: \mathbb{C}^{n} \rightarrow \mathbb{C}^{p}$ ), they showed that $K_{\infty}(f)$ is a closed semi-algebraic set (respectively, a closed algebraic set) of dimension at most $p-1$. In particular, this shows that $B(f)$ has volume zero.

Jelonek [24] used another condition, which turns out to be equivalent to Rabier condition and to Gaffney condition. We call that condition Jelonek condition. From this last condition, Jelonek [24, Theorem 3.1] gave a more direct proof of the inclusion: $B(f) \subset(f(\operatorname{Sing} f) \cup$ $K_{\infty}(f)$, where $f: \mathbb{K}^{n} \rightarrow \mathbb{K}^{p}, \mathbb{K}=\mathbb{R}, \mathbb{C}$, is a smooth mapping.

We remark that, for functions (i.e. $p=1$ ), Rabier, Gaffney, Kuo-KOS and Jelonek conditions coincide with the Malgrange condition and it was established by Jelonek [24], Kurdyka, Orro and Simon [29] that these generalizations of Malgrange condition for mappings are equivalent (see Definition 2.3.1 and Remark 2.3.4 for details on Rabier, Gaffney, Kuo-KOS 
and Jelonek conditions). In other words, these four conditions yield the same set, which we denote in this thesis by $\mathcal{N}_{\infty}(f)$, of non-regular values at infinity (i.e. $K_{\infty}(f)=A_{G_{\infty}}(f)=$ $\mathcal{N}_{\infty}(f)$, where $K_{\infty}(f), A_{G_{\infty}}(f)$ were above mentioned) (see $\S 2.3$ for details).

In summary, for $f: \mathbb{K}^{n} \rightarrow \mathbb{K}^{p}\left(C^{2}\right.$ semi-algebraic for $\mathbb{K}=\mathbb{R}$ and polynomial mapping for $\mathbb{K}=\mathbb{C}$ ), we have for these four conditions a fibration theorem (i.e. $\left.B(f) \subset\left(f(\operatorname{Sing} f) \cup \mathcal{N}_{\infty}(f)\right)\right)$ and a structure theorem (i.e. $\mathcal{N}_{\infty}(f)$ is semi-algebraic (or algebraic in the complex setting) of dimension at most $p-1)$. These two results mean an asymptotic Morse-Sard type theorem for these four conditions together with a fibration theorem for non-proper mappings $f: \mathbb{K}^{n} \rightarrow \mathbb{K}^{p}$.

The central objects in this thesis are the t-regularity, a geometric grounded condition, and the $\rho_{E}$-regularity, a Milnor-type condition, in the setting of semi-algebraic mappings $f: \mathbb{R}^{n} \rightarrow \mathbb{R}^{p}$ and complex polynomial mappings $f: \mathbb{C}^{n} \rightarrow \mathbb{C}^{p}$.

This thesis is essentially structured in two parts: firstly, we consider semi-algebraic mappings $f: \mathbb{R}^{n} \rightarrow \mathbb{R}^{p}$ and complex polynomial mappings $f: \mathbb{C}^{n} \rightarrow \mathbb{C}^{p}$ (Chapters 1, 2 and 3). After, we consider polynomial mappings $f: \mathbb{R}^{n} \rightarrow \mathbb{R}^{p}$ (Chapters 4 and 5 ).

For $C^{1}$ semi-algebraic mappings $f: \mathbb{R}^{n} \rightarrow \mathbb{R}^{p}$ and complex polynomial mappings $f: \mathbb{C}^{n} \rightarrow$ $\mathbb{C}^{p}$, Theorem 2.4.8 and Corollary 2.4.9 state that $t$-regularity is equivalent to the asymptotic conditions used in Rabier [48], Gaffney [18], Kurdyka, Orro and Simon [29] and Jelonek [24]. This equivalence represents a geometric interpretation of the asymptotic conditions and it is an extension of the equivalence proved for $p=1$ in Parusiński [42] and Siersma and Tibăr [49], and an extension of the equivalence obtained for a class of polynomial mappings $f: \mathbb{C}^{n} \rightarrow \mathbb{C}^{p}$ in Gaffney [18].

The $\rho_{E}$-regularity enables one to define the set of asymptotic non $\rho_{E}$-regular values $S(f) \subset$ $\mathbb{K}^{p}$, and the set $A_{\rho_{E}}:=f(\operatorname{Sing} f) \cup S(f)$.

We pursue by showing (Proposition 2.5.4 and Proposition 2.5.5) that $t$-regularity implies $\rho_{E}$-regularity, extending a result proved for $p=1$ in Tibăr [55] (see also Păunescu and Zaharia [45]). In particular, these propositions imply $S(f) \subset \mathcal{N}_{\infty}(f)$. We note that this inclusion may be strict, cf Example 2.5.6.

Then, for $C^{2}$ semi-algebraic mappings $f: \mathbb{R}^{n} \rightarrow \mathbb{R}^{p}$ (respectively, polynomial mappings $f: \mathbb{C}^{n} \rightarrow \mathbb{C}^{p}$ ), we prove (Theorem 3.1.1(b) and Theorem 3.1.8) that $S(f)$ and $A_{\rho_{E}}$ are real closed semi-algebraic sets of dimension at most $p-1$ (respectively real dimension at most $2 p-2)$. These refines the results of Kurdyka, Orro and Simon [29]. In particular, the key result $\operatorname{dim} \mathcal{N}_{\infty}(f) \leq p-1$ of Kurdyka, Orro and Simon [29] is superseded by $\operatorname{dim} S(f) \leq p-1$. Our proof is of a completely different flavour and is based on the existence of partial Thom stratifications at infinity (which has been introduced by Tibăr [54, Definition 2.1] for $p=1$ ), see Definition 3.1.3 for the notion of partial Thom stratification at infinity.

Moreover, Theorem 3.1.1(a) and Theorem 3.1.8 show that there is a locally trivial fibration induced by $f$ outside $A_{\rho_{E}}$. These fibration results are based on a fibration theorem at infinity (cf. Proposition 3.1.5), i.e. holding in the complement of a sufficiently large ball. These refine the fibration results of Rabier [48] (in the case of semi-algebraic mappings and complex 
polynomial mappings), Jelonek [24] and Gaffney [18], where the set $\mathcal{N}_{\infty}(f)$ (equivalently $K_{\infty}(f), A_{G_{\infty}}(f)$, above mentioned) are superseded by $S(f)$.

Therefore, Theorem 3.1.1 and Theorem 3.1.8 represent asymptotic Morse-Sard type theorems with the set of non $\rho_{E}$-regular values $S(f)$, which refine the results obtained with the set $\mathcal{N}_{\infty}(f)$ (equivalently with the sets above mentioned $K_{\infty}(f)$ and $A_{G_{\infty}}(f)$ ).

In Chapter 4, we restrict our investigation to two classes of polynomial mappings $f: \mathbb{R}^{n} \rightarrow$ $\mathbb{R}^{p}$ : the class of fair polynomial mappings (Definition 4.2.4) and the class of Newton nondegenerate polynomial mappings (Definition 4.3.3). Note that, since polynomial mappings are semi-algebraic mappings, the results in Chapters 1, 2 and 3 also hold for these two classes of polynomial mappings $f: \mathbb{R}^{n} \rightarrow \mathbb{R}^{p}$.

Following Gaffney [18], we define the class of fair polynomial mappings (Definition 4.2.4) and we give an algebraic interpretation of the $t$-regularity in terms of the theory of real integral closure of modules (Proposition 4.2.5). This interpretation allows one to prove in a different way the equivalence (above mentioned): " $t$-regularity $\Leftrightarrow$ Gaffney (or Jelonek, or Kuo-KOS, or Rabier) condition" (Theorem 4.2.6 and Remark 4.2.8). These are the real counterparts of Gaffney's results [18].

After, we introduce a Newton non-degeneracy condition at infinity for polynomial mappings $f: \mathbb{R}^{n} \rightarrow \mathbb{R}^{p}$. We have worked out this problem together with the Phd student Chen [6]. He develops in his thesis the notion of non-degeneracy for mixed polynomial mappings (see Chen [6] and Chen, Dias and Tibăr [7]).

This non-degeneracy (Definition 4.3.3) is designed to study the bifurcation values of $f$ and specially the case of non-convenient polynomial mappings (Definition 4.3.1).

Under this non-degeneracy condition, we obtain in Theorem 4.3.8 an approximation of the bifurcation values of $f$ in terms of the critical values of restrictions $f_{\triangle}$ to certain faces of the Newton polyhedron of the components of $f$. Results like this have been obtained recently for mixed functions by Chen and Tibăr [8, Theorem 1.1 (a)], and previously by Némethi and Zaharia [40, Theorem 2] for complex polynomial functions $f: \mathbb{C}^{n} \rightarrow \mathbb{C}$ (see also Chen, Dias and Tibăr [7] and Phd thesis of Chen [6]).

We present some consequences of Theorem 4.3.8. In special, we show in Corollary 4.3.11 that if $f=\left(f_{1}, \ldots, f_{p}\right)$ is Newton non-degenerate and each $f_{i}, i=1, \ldots, p$, is convenient then $B(f) \subset f(\operatorname{Sing} f)$. Similar results had been obtained by Broughton [4, Proposition 3.4] for polynomial functions $f: \mathbb{C}^{n} \rightarrow \mathbb{C}$ and more recently by Chen and Tibăr [8, Corollary 4.1] for mixed functions.

We compare this non-degeneracy condition with the non-degeneracy condition considered by Bivià-Ausina [2] and with the classical Khovanskii non-degeneracy condition [26] (see our §4.3.3). In special, we show that in the case $n>p$ and $f_{i}$ convenient, $i=1, \ldots, p$, our definition of non-degeneracy is more general than the one considered by Bivià-Ausina [2], and that, for $n=k$, the two definitions are equivalent, cf Proposition 4.3.16.

Connected to the study of $B(f)$, we study the topological structure of the fibres of $f$ 
and the surjectivity and injectivity of mappings $f: \mathbb{R}^{n} \rightarrow \mathbb{R}^{n}$. These questions are briefly discussed in Chapter 5 as follows: in Proposition 5.3.5, we present a formula for the Euler characteristic of regular fibres of $f$. In Proposition 5.2.1, we discuss a criterion for a local diffeomorphism to be a global diffeomorphism. As a consequence of Corollary 4.3.11, we obtain that if $f=\left(f_{1}, \ldots, f_{p}\right)$ is Newton non-degenerate, each $f_{i}, i=1, \ldots, p$, is convenient and $f$ is a local diffeomorphism then $f$ is a global diffeomorphism (Proposition 5.2.1).

This thesis is organised as follows:

Chapter 1: In $\S 1.2$, we briefly recall the definitions of equivalent norms on the space of linear mappings from $\mathbb{K}^{n}$ to $\mathbb{K}^{p}$ (this last space will be denoted here by $\mathcal{L}\left(\mathbb{K}^{n}, \mathbb{K}^{p}\right)$ ). In $\S 1.3$, we present four functions defined on $\mathcal{L}\left(\mathbb{K}^{n}, \mathbb{K}^{p}\right)$ : Rabier function, Kuo function, Gaffney function and Jelonek function. We discuss their relations and properties. In $§ 1.4$, following Jelonek $[24,25]$, we present these four functions on $\mathcal{L}\left(V, \mathbb{K}^{p}\right)$, where $V$ is a linear subspace of $\mathbb{K}^{n}$.

Chapter 2: In $\S 2.2$, we present some well-known facts about the bifurcation set of polynomial functions $f: \mathbb{K}^{n} \rightarrow \mathbb{K}$ and discuss some regularity conditions at infinity which have been used to approximate $B(f)$. In $\S 2.3$, we discuss some important results from the works of Rabier [48], Gaffney [18], Jelonek [24, 25] and Kurdyka, Orro and Simon [29] for semi-algebraic mappings $f: \mathbb{R}^{n} \rightarrow \mathbb{R}^{p}$ and polynomial mappings $f: \mathbb{C}^{n} \rightarrow \mathbb{C}^{p}$.

In $\S 2.4$, we consider the $t$-regularity in the setting of $C^{1}$ semi-algebraic mappings $f: \mathbb{R}^{n} \rightarrow$ $\mathbb{R}^{p}$ and polynomial mappings $f: \mathbb{C}^{n} \rightarrow \mathbb{C}^{p}$. We reformulate Rabier, Kuo-KOS, Gaffney and Jelonek conditions in a localized version on a point of the boundary at infinity of the graph of $f$ in $\mathbb{P}^{n} \times \mathbb{K}^{p}$. We compare these conditions to the $t$-regularity. In $\S 2.5$, we consider the $\rho_{E}$-regularity and show that $t$-regularity implies $\rho_{E}$-regularity.

Chapter 3: We obtain a fibration theorem and a structure theorem for $C^{2}$ semi-algebraic mappings $f: X \rightarrow \mathbb{R}^{p}$, where $X$ is semi-algebraic, and for polynomial mappings $f: \mathbb{C}^{n} \rightarrow \mathbb{C}^{p}$. We discuss an example of a family of polynomial functions in $\S 3.3$.

Chapter 4: We consider two classes of polynomial mappings $f: \mathbb{R}^{n} \rightarrow \mathbb{R}^{p}$ : the class of fair polynomial mappings (\$4.2) and the class of Newton non-degenerate polynomial mappings (§4.3). For fair polynomial mappings, we give an algebraic interpretation of $t$-regularity in terms of the theory of real integral closure of modules. For Newton non-degenerate polynomial mappings, we give an approximation to the set $B(f)$ in terms of a set that depends only of the Newton polyhedron of $f$. We also present some consequences of this last result.

Chapter 5: We briefly discuss some simple consequences from the previous chapters. In §5.1, we discuss the equivalence $t$-regularity $\Leftrightarrow$ Rabier (equivalently Gaffney, Kuo-KOS, Jelonek) condition for mappings $f: X \rightarrow \mathbb{K}^{p}$, where $X \subset \mathbb{K}^{n}$ is a smooth affine variety. In $\S 5.2$, we discuss the problem of bijectivity of semi-algebraic mappings. We present in $\S 5.3$ a formula to compute the Euler characteristic of regular fibres of polynomial mappings $f: \mathbb{R}^{n} \rightarrow \mathbb{R}^{n-1}$. 


\section{Preliminaries}

\section{Contents}

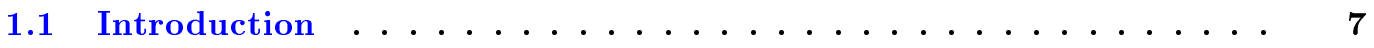

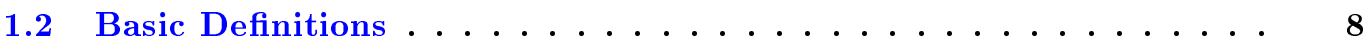

1.3 Rabier, Gaffney, Kuo, Jelonek functions . . . . . . . . . . . . 10

1.4 Relative Functions . . . . . . . . . . . . . . . . . . . 14

\section{$1.1 \quad$ Introduction}

We denote by $\mathcal{L}(V, W)$ the set of linear mappings from $V$ to $W$, where $V, W$ are normed finite dimensional vector spaces over $\mathbb{R}$ or $\mathbb{C}$. For simplicity, sometimes we will denote $\mathcal{L}(V, \mathbb{K})$ simply by $V^{*}$.

Let $f: \mathbb{K}^{n} \rightarrow \mathbb{K}^{p}$ be a mapping of class $C^{2}, n \geq p, \mathbb{K}=\mathbb{R}$ or $\mathbb{C}$. An approach to study the bifurcation values of $f$ is to assume on the affine space $\mathbb{K}^{n}$ an asymptotic behaviour of the fibres of $f$. Through this assumption, we can use the Jacobian matrix of $f$ to construct vector fields which trivialize $f$. More precisely, one considers some specific function " $\beta$ " defined on $\mathcal{L}\left(\mathbb{K}^{n}, \mathbb{K}^{p}\right)$ and requires a regularity condition on a point $t_{0} \in \mathbb{K}^{p} \backslash f(\operatorname{Sing} f)$ like the folowing:

$$
\exists \delta>0 \text { such that } \delta<\|x\| \beta(\mathrm{D} f(x)) \text {, as }\|x\| \rightarrow \infty \text { and } f(x) \rightarrow t_{0},
$$

where $\mathrm{D} f(x)$ denotes the Jacobian matrix of $f$ at $x$.

Then, one constructs a vector field from the Jacobian matrix of $f$ and, by the condition (1.1), one can show that the flow of this vector field trivializes $f$ on a neighbourhood $U$ of $t_{0}$. In this way, the condition (1.1) yields a set $\mathcal{N} \mathcal{R}_{\infty}(f)$ of "non- $\beta$-regular values", so that one obtains the inclusion: $B(f) \subset \mathcal{N} \mathcal{R}_{\infty}(f) \cup f(\operatorname{Sing} f)$.

This method has been used by many authors with different functions $\beta$ to study the bifurcation values of polynomial functions $f: \mathbb{K}^{n} \rightarrow \mathbb{K}$. See for instance Broughton [4, Proposition 2], Némethi and Zaharia [40, Lemma 3], Parusiński [42, Lemma 1.2], Siersma and Tibăr [49, page 780], and see Tibăr [56, Chapter 1] for results comparing these functions.

For mappings, Rabier [48], Gaffney [18] and Jelonek [24, 25] have considered the above approach to obtain fibration theorems for mappings in different contexts:

- Rabier [48] considers a function $\nu$ defined on $\mathcal{L}(X, Y)$, where $X, Y$ are Banach spaces over $\mathbb{R}$ or $\mathbb{C}$. Using the function $\nu$, he defines the notion of "strong submersion" for 
mappings $f: M \rightarrow N$, where $M$ and $N$ are Finsler manifolds. On additional hypothesis on $M, N$ and some technical assumption, Rabier proves fibration theorems for strong submersion mappings $([48, \S 4, \S 5, \S 6])$.

- Gaffney [18] studies complex polynomial mappings $f: \mathbb{C}^{n} \rightarrow \mathbb{C}^{p}$. He defines another function on $\mathcal{L}\left(\mathbb{C}^{n}, \mathbb{C}^{p}\right)$, which we denote here by $\gamma$. Assuming a regular condition like (1.1) with the function $\gamma$, Gaffney obtains a fibration theorem for complex polynomial mappings, see [18, Theorem 18].

- In [24, 25], Jelonek defines another function, that we denote by $\zeta$. He compares the functions $\nu, \gamma, \zeta$ and other functions. Via these relations, he gives a direct proof of Rabier's fibration theorem for smooth mapping $f: \mathbb{K}^{n} \rightarrow \mathbb{K}^{p}$ ([24, Theorem 3.1]). In [25, Proposition 3.1], Jelonek obtains a fibration theorem for smooth mapping $f: X \rightarrow \mathbb{K}^{p}$, where $X$ is a smooth affine variety over $\mathbb{K}$.

- Kurdyka, Orro and Simon also consider the Rabier function in [29] and obtain some relations between this function and other functions. In special, they obtain an equivalence between the Rabier function and the Kuo function $\kappa$ (see [29, §2.2] for this equivalence and Kuo [27, page 116] for the Kuo function $\kappa$, see also our subsection 1.3).

In Chapter 2, we obtain for semi-algebraic mappings a geometric interpretation (called $t$-regularity) for condition (1.1) applied to the functions $\nu, \gamma, \kappa, \zeta$, or to any equivalent function to them. Details about this interpretation, the importance of these functions in our work and in the study of bifurcation values will be explained in Chapter 2. More details about the works [18, Gaffney], [24, 25, Jelonek], [29, Kurdyka, Orro and Simon] and [48, Rabier] will be discussed specially in $\S 2.1$.

In this chapter we concentrate on these functions, some of their relations and properties, which will be useful to define regularity conditions and to compute examples. Firstly, in the preliminary section $\S 1.2$, we give the definition of norm on the space $\mathcal{L}(V, W)$, where $V, W$ are finite dimensional vector spaces over $\mathbb{R}$ or $\mathbb{C}$. We present three equivalent definitions of norm for a functional $A \in \mathcal{L}\left(\mathbb{K}^{n}, \mathbb{K}\right)$. These definitions will be used throughout the text to compute and to define the above mentioned functions.

In $\S 1.3$, we present four functions defined on $\mathcal{L}\left(\mathbb{K}^{n}, \mathbb{K}^{p}\right)$ : the Rabier function, the Kuo function, the Gaffney function and the Jelonek function. We discuss their relations and properties. In this subsection, these functions will be defined on $\mathcal{L}\left(\mathbb{K}^{n}, \mathbb{K}^{p}\right)$. Following Jelonek $[24,25]$, in $\S 1.4$, we present these functions on $\mathcal{L}\left(V, \mathbb{K}^{p}\right)$, where $V$ is a linear subspace of $\mathbb{K}^{n}$.

\subsection{Basic Definitions}

Let $V, W$ be normed finite dimensional vector spaces over $\mathbb{K}$, where $\mathbb{K}=\mathbb{R}$ or $\mathbb{C}$. As before, we denote by $\mathcal{L}(V, W)$ the set of linear mappings from $V$ to $W$ and, sometimes, we denote $\mathcal{L}(V, \mathbb{K})$ simply by $V^{*}$. We define the operator norm on $A \in \mathcal{L}(V, W)$ as follows: 
Definition 1.2.1. Let $V$ and $W$ be normed finite dimensional vector spaces over $\mathbb{K}$, where $\mathbb{K}=\mathbb{R}, \mathbb{C}$. The operator norm of $A \in \mathcal{L}(V, W)$, denoted by $\|$.$\| , is defined as:$

$$
\|A\|:=\max \{\|A(x)\| ; x \in V \text { and }\|x\|=1\} .
$$

The above definition can be seen in Folland [15, page 145]. Another norm on $\mathcal{L}\left(\mathbb{K}^{n}, \mathbb{K}\right)$ is defined in the following way:

Definition 1.2.2. Let $A \in \mathcal{L}\left(\mathbb{K}^{n}, \mathbb{K}\right)$. Define the vector $A(\mathrm{e}):=\left(A\left(\mathrm{e}_{1}\right), \ldots, A\left(\mathrm{e}_{n}\right)\right)$, where $\mathrm{e}_{i}$ denotes the vector of $\mathbb{K}^{n}$ with 1 in the $i$-th coordinate and $0^{\prime}$ s elsewhere. We define the norm $\|\cdot\|_{1}$ of $A$ as follows:

$$
\|A\|_{1}:=\|A(\mathrm{e})\|
$$

We remember that the norms $\|.\|_{1}$ and $\|.\|_{2}$ are equivalent if there exist positive constants $c_{1}, c_{2}$ such that $c_{1}\|\cdot\|_{1} \leq\|\cdot\|_{2} \leq c_{2}\|\cdot\|_{1}$. The next well-known result can be seen in Young [60, Theorem 6.8].

Lemma 1.2.3. On the vector space $\mathcal{L}\left(\mathbb{K}^{n}, \mathbb{K}\right)$, the norms from Definition 1.2 .1 and Definition 1.2.2 are equivalent.

Sometimes we consider mappings $f: X \rightarrow \mathbb{K}^{p}$, where $X$ is a smooth variety of $\mathbb{K}^{n}$. So, the next definition and the next lemma will be useful for us:

Definition 1.2.4. Let $A \in \mathcal{L}\left(\mathbb{K}^{n}, \mathbb{K}\right)$ and let $V \subset \mathbb{K}^{n}$ be a linear subspace of $\mathbb{K}^{n}$. We denote by $A_{\mid V}$ the restriction of $A$ to $V$. Consider $V^{\perp}:=\left\{w \in \mathbb{K}^{n} \mid\langle w, v\rangle=0, \forall v \in V\right\}$. We define the norm $\|.\|_{3}$ of $A_{\mid V}$ in $\mathcal{L}(V, \mathbb{K})$ as follows:

$$
\left\|A_{\mid V}\right\|_{3}:=\min \left\{\|A(\mathrm{e})+w\| ; w \in V^{\perp}\right\}
$$

where $A(\mathrm{e})$ is defined as in the Definition 1.2.2.

Lemma 1.2.5. Let $A \in \mathcal{L}\left(\mathbb{K}^{n}, \mathbb{K}\right)$ and let $V \subset \mathbb{K}^{n}$ be a linear subspace of $\mathbb{K}^{n}$. The norms of $A_{\mid V}$ defined in Definition 1.2.1 and Definition 1.2.4 are equivalent.

Proof. Let $A$ be an arbitrary element of $\mathcal{L}\left(\mathbb{K}^{n}, \mathbb{K}\right)$. We need to find positive constants $c_{1}$ and $c_{2}$, independent of $A$, such that $c_{1}\left\|A_{\mid V}\right\| \leq\left\|A_{\mid V}\right\|_{3} \leq c_{2}\left\|A_{\mid V}\right\|$. In fact, we will show that $c_{1}=c_{2}=1$, which implies $\left\|A_{\mid V}\right\|_{3}=\left\|A_{\mid V}\right\|$.

Since $A$ is a linear mapping, one can write $A(v)=\sum_{i=1}^{n} v_{i} A\left(\mathrm{e}_{i}\right)=\langle v, A(\mathrm{e})\rangle$, for any vector $v=\left(v_{1}, \ldots, v_{n}\right) \in V$. Thus, for any vector $v \in V$, with $\|v\|=1$, and for any vector $w \in V^{\perp}$ one has:

$$
\|A(v)\|=\|\langle v, A(\mathrm{e})\rangle\|=\|\langle v, A(\mathrm{e})\rangle+\langle v, w\rangle\|=\|\langle v, A(\mathrm{e})+w\rangle\| \leq\|v\|\|A(\mathrm{e})+w\|,
$$


where the second equality follows from the fact that $w \in V^{\perp}$, i.e. $\langle v, w\rangle=0$, and the last inequality comes from the Cauchy-Schwarz inequality.

Since we have supposed $\|v\|=1$, the inequality (1.5) gives us $\|A(v)\| \leq\|A(\mathrm{e})+w\|$. Then, since the vectors $v, w$ are arbitrary elements, the inequality $\|A(v)\| \leq\|A(\mathrm{e})+w\|$ implies:

$$
\left\|A_{\mid V}\right\| \leq\left\|A_{\mid V}\right\|_{3}
$$

To show the other inequality, i.e. $\left\|A_{\mid V}\right\|_{3} \leq\left\|A_{\mid V}\right\|$, we observe that since $V \oplus V^{\perp}=\mathbb{K}^{n}$, there exist $\mathrm{v}_{1} \in V$ and $\mathrm{w}_{1} \in V^{\perp}$ such that $A(\mathrm{e})=\mathrm{v}_{1}+\mathrm{w}_{1}$. This implies that for any $v \in V$, one has $A(v)=\langle v, A(\mathrm{e})\rangle=\left\langle v, \mathrm{v}_{1}+\mathrm{w}_{1}\right\rangle=\left\langle v, \mathrm{v}_{1}\right\rangle$, where the last equality follows from the fact that $\mathrm{w}_{1} \in V^{\perp}$.

So, if $\mathrm{v}_{1}=0$ one has $A_{\mid V} \equiv 0$ and $A(\mathrm{e})=\mathrm{w}_{1}$, which implies $\left\|A_{\mid V}\right\|=0$ and $\left\|A_{\mid V}\right\|_{1}=0$. Consequently the inequality $\left\|A_{\mid V}\right\|_{1} \leq\left\|A_{\mid V}\right\|$ is true if $\mathrm{v}_{1}=0$.

On the other hand, if $\mathrm{v}_{1} \neq 0$ we define the vector $z:=\frac{\mathrm{v}_{1}}{\left\|\mathrm{v}_{1}\right\|}$. Then $z \in V,\|z\|=1$ and $A(z)=\langle z, A(\mathrm{e})\rangle=\left\langle z, \mathrm{v}_{1}+\mathrm{w}_{1}\right\rangle=\left\langle z, \mathrm{v}_{1}\right\rangle$, where the last equality follows from the fact that $\mathrm{w}_{1} \in V^{\perp}$. So, by definition of vector $z$, one obtains $A(z)=\left\langle z, \mathrm{v}_{1}\right\rangle=\left\|\mathrm{v}_{1}\right\|$. Since $\|z\|=1$, one has $\|A(z)\|=\left\|\mathrm{v}_{1}\right\| \leq\left\|A_{\mid V}\right\|$, where the last inequality follows from the definition of $\left\|A_{\mid V}\right\|$.

To finish, we observe that $A(\mathrm{e})-\mathrm{w}_{1}=\mathrm{v}_{1}$, with $\mathrm{w}_{1} \in V^{\perp}$, which by definition of $\left\|A_{\mid V}\right\|_{1}$ implies $\left\|A_{\mid V}\right\|_{3} \leq\left\|\mathrm{v}_{1}\right\|$. So, we conclude $\left\|A_{\mid V}\right\|_{3} \leq\left\|\mathrm{v}_{1}\right\| \leq\left\|A_{\mid V}\right\|$, which implies $\left\|A_{\mid V}\right\|_{3} \leq$ $\left\|A_{\mid V}\right\|$.

Therefore, by the last inequality and by inequality (1.6), one obtains $\left\|A_{\mid V}\right\|=\left\|A_{\mid V}\right\|_{3}$, and this finish the proof.

\subsection{Rabier, Gaffney, Kuo, Jelonek functions}

In this section, we define the Rabier function, the Kuo function, the Gaffney function and the Jelonek function. We also present results from Jelonek [24, 25], Kuo [27] and Kurdyka, Orro, Simon [29], which describe some relations and properties among these functions. We begin with:

\section{Rabier function}

Consider $V, W$ normed finite dimensional vector spaces over $\mathbb{R}$ or $\mathbb{C}$. Given $A \in \mathcal{L}(V, W)$, we denote by $A^{*} \in \mathcal{L}\left(W^{*}, V^{*}\right)$ the adjoint operator induced by $A$ and one has:

Definition 1.3.1 ([48, page 651]). The Rabier function, denoted by $\nu$, is defined as follows:

$$
\nu(A):=\inf \left\{\left\|A^{*}(\varphi)\right\| ; \varphi \in W^{*} \text { and }\|\varphi\|=1\right\} .
$$

Kurdyka, Orro and Simon [29, Section 2] and Rabier [48, Section 2] discuss interesting properties of the Rabier function. In particular, Kurdyka, Orro, Simon [29, Proposition 2.2] show that the above function measures the distance of $A$ to the set $\Sigma$, where $\Sigma:=\{B \in$ 
$\mathcal{L}(V, W) \mid B$ is not onto\}. Consequently, this gives another characterization for the function $\nu$ in terms of the equality: $\nu(A)=\operatorname{dist}(A ; \Sigma)$. In particular, from this characterization one has $\nu(A)=0$ if and only if $A$ is not onto.

\section{Kuo function}

To define the Kuo function we need the following:

Definition 1.3.2 ([27, page 116]). Let $v_{1}, \ldots, v_{p}$ be vectors in $\mathbb{K}^{n}$. The Kuo distance between these vectors is defined by the following function:

$$
\kappa\left(v_{1}, \ldots, v_{p}\right):=\min _{1 \leq i \leq p}\left\{\operatorname{dist}\left(v_{i} ;\left\langle\left(v_{j}\right)_{j \neq i}\right\rangle\right)\right\}
$$

where $\left\langle\left(v_{j}\right)_{j \neq i}\right\rangle$ denotes the space generated by the vectors $\left\{v_{j} \mid j \neq i\right\}$.

Consider vectors $v_{1}, \ldots, v_{p} \in \mathbb{K}^{n}$. In some sense, the Kuo distance measures the linear dependency of the vectors $v_{1}, \ldots, v_{p}$. In fact, from equality $(1.8)$ we have $\kappa\left(v_{1}, \ldots, v_{p}\right)=0$ if and only if the vectors $v_{1}, \ldots, v_{p}$ are linearly dependent.

From the Kuo distance one has:

Definition 1.3.3 $\left(\left[27\right.\right.$, page 116]). Let $A=\left(A_{1}, \ldots, A_{p}\right) \in \mathcal{L}\left(\mathbb{K}^{n}, \mathbb{K}^{p}\right)$, with $n \geq p$. Denote by $\eta_{i}$ the gradient of $A_{i}, i=1, \ldots, p$. We set the Kuo function, denoted also by $\kappa$, as follows:

$$
\kappa(A):=\kappa\left(\eta_{1}, \ldots, \eta_{p}\right),
$$

where $\kappa\left(\eta_{1}, \ldots, \eta_{p}\right)$ is defined as in the Definition 1.3.2.

It is interesting to remark that the Kuo function has been used by many authors in order to study a local problem, the $v$-sufficiency of jets of $C^{r}$ map germs $f:\left(\mathbb{R}^{n}, 0\right) \rightarrow\left(\mathbb{R}^{p}, 0\right)$. In special, Kuo [27] gives a characterization for the $v$-sufficiency of jets in terms of a Łojasiewicz type inequality, which depends of the Kuo function.

On the other hand, the Rabier function has been used by Rabier [48] to give conditions to obtain fibration theorems for maps and to study global problems. The link between the functions of Rabier and Kuo is due to the work of Kurdyka, Orro, Simon [29], that we present in the next:

Lemma 1.3.4 ([29, Proposition 2.6]). Let $A=\left(A_{1}, \ldots, A_{p}\right) \in \mathcal{L}\left(\mathbb{K}^{n}, \mathbb{K}^{p}\right), n \geq p$. Denote by $\eta_{i}$ the gradient of $A_{i}$, for $i=1, \ldots, p$. The following inequalities hold:

$$
\nu(A) \leq \kappa\left(\eta_{1}, \ldots, \eta_{p}\right) \leq \sqrt{p} \nu(A) .
$$




\section{Gaffney function}

Let $[\mathbf{a}]$ be a $p \times n$ matrix and let $k$ be a positive integer with $k \leq \min \{p, n\}$. A minor of [a] of size $k$ is the determinant of a $k \times k$ matrix obtained from [a] by deleting $p-k$ rows and $n-k$ columns. When $k=\min \{p, n\}$, the minors of size $k$ are called maximal minors of $[\mathbf{a}]$. Under these notations and definitions one has:

Definition 1.3.5 ([18, page 158]). Consider $A \in \mathcal{L}\left(\mathbb{K}^{n}, \mathbb{K}^{p}\right), n \geq p$. Denote by $[\mathbf{a}]$ the matrix of $A$. Let $I=\left(i_{1}, \ldots, i_{p}\right)$ and $J=\left(j_{1}, \ldots, j_{p-1}\right)$ be multi-indices with $i_{1}<\ldots<i_{p}$ and $j_{1}<\ldots<j_{p-1}$. We denote by $M_{I}(A)$ the maximal minor of [a] formed from the columns indexed by $I$ and we denote by $M_{J, j}(A)$ the minor of $[\mathbf{a}]$ of size $(p-1)$ using the columns indexed by $J$, and all the rows of the matrix [a] except for the $j$ th row. By convention, if $p=1$ then $M_{J, j}(A)=1$. The Gaffney function $\gamma$ is defined as:

$$
\gamma(A):=\frac{\left(\sum_{I}\left\|M_{I}(A)\right\|^{2}\right)^{1 / 2}}{\left(\sum_{J, j}\left\|M_{J, j}(A)\right\|^{2}\right)^{1 / 2}} .
$$

If the function $\gamma(A)$ is not defined (i.e., if $\sum_{J, j}\left\|M_{J, j}(A)\right\|^{2}=0$ ) we set $\gamma(A)=0$.

We remark that Gaffney [18] defined the above function in the complex case, i.e. in $\mathcal{L}\left(\mathbb{C}^{n}, \mathbb{C}^{p}\right)$. With the same expression of $(1.11)$, Jelonek [24, Definition 2.2] considered the above function for $\mathcal{L}\left(\mathbb{K}^{n}, \mathbb{K}^{p}\right)$.

The next lemma, due to Jelonek [24], gives a relation between $\kappa$ and $\gamma$ :

Lemma 1.3.6 ([24, Proposition 2.3]). There exist positive constants $c_{1}, c_{2}$ such that, for any $A \in \mathcal{L}\left(\mathbb{K}^{n}, \mathbb{K}^{p}\right)$, the following inequalities hold:

$$
c_{1} \kappa(A) \leq \gamma(A) \leq c_{2} \kappa(A),
$$

where $\kappa$ is as in (1.9) and $\gamma$ is as in (1.11).

\section{Jelonek function}

Jelonek [24] defines another function on $A \in \mathcal{L}\left(\mathbb{K}^{n}, \mathbb{K}^{p}\right)$, where $n \geq p$, as follows:

Definition 1.3.7 ([24, Definition 2.3]). Consider $A \in \mathcal{L}\left(\mathbb{K}^{n}, \mathbb{K}^{p}\right)$, where $n \geq p$. Denote by $[\mathbf{a}]$ the matrix of $A$. Let $I=\left(i_{1}, \ldots, i_{p}\right)$ and $J=\left(j_{1}, \ldots, j_{p-1}\right)$ be multi-indices with $i_{1}<\ldots<i_{p}$ and $j_{1}<\ldots<j_{p-1}$. As in Definition 1.3.5, one denotes by $M_{I}(A)$ the maximal minor of [a] formed from the columns indexed by $I$ and by $M_{J, j}(A)$ the minor of [a] of size $(p-1)$ using the columns indexed by $J$, and all the rows of the matrix [a] except for the $j$ th row. 
By convention, if $p=1$ then $M_{J, j}(A)=1$. The Jelonek function $\zeta$ is defined as:

$$
\zeta(A):=\max _{I}\left\{\min _{J \subset I, 1 \leq j \leq p} \frac{\left\|M_{I}(A)\right\|}{\left\|M_{J, j}(A)\right\|}\right\} \cdot 1
$$

From the above definition, we have that $\zeta$ also depends on the minors of the matrix [a] and we have the following relation:

Lemma 1.3.8 ([24, Proposition 2.4]). Consider $A \in \mathcal{L}\left(\mathbb{K}^{n}, \mathbb{K}^{p}\right)$. Then there exist positive constants $c_{1}, c_{2}$ such that

$$
c_{1} \zeta(A) \leq \gamma(A) \leq c_{2} \zeta(A)
$$

The functions $\nu, \kappa, \gamma$ and $\zeta$ are not pseudo-norms on the space $\mathcal{L}\left(\mathbb{K}^{n}, \mathbb{K}^{p}\right)$, for $n \geq p>1$ (they do not satisfy the triangle inequality property). We can see this in the following:

EXAmple 1.3.9. Consider the linear mappings $A_{1}(x, y, z):=(x, 0)$ and $A_{2}(x, y, z):=(0, z)$, with $A_{1}, A_{2} \in \mathcal{L}\left(\mathbb{K}^{3}, \mathbb{K}^{2}\right)$. Then $\nu\left(A_{i}\right)=\kappa\left(A_{i}\right)=\gamma\left(A_{i}\right)=\zeta\left(A_{i}\right)=0$, for $i=1,2$. On the other hand, we have $\nu\left(A_{1}+A_{2}\right)=\kappa\left(A_{1}+A_{2}\right)=\zeta\left(A_{1}+A_{2}\right)=1$ and $\gamma\left(A_{1}+A_{2}\right)=\sqrt{2} / 2$, which implies that the triangle inequality is not satisfied for the functions $\nu, \kappa, \gamma, \zeta$ on the space $\mathcal{L}\left(\mathbb{K}^{3}, \mathbb{K}^{2}\right)$. Therefore these four functions are not pseudo-norms on $\mathcal{L}\left(\mathbb{K}^{3}, \mathbb{K}^{2}\right)$.

Furthermore, we can construct similar examples to show that these functions are not pseudo-norms on the space $\mathcal{L}\left(\mathbb{K}^{n}, \mathbb{K}^{p}\right), n \geq p>1$. Consequently, since any norm is a pseudonorm, we have that these functions are not norms on $\mathcal{L}\left(\mathbb{K}^{n}, \mathbb{K}^{p}\right)$, with $n \geq p>1$. However, in the case $p=1$ one has:

Remark 1.3.10. $\nu(A)=\kappa(A)=\gamma(A)=\|A\|$, for any $A \in \mathcal{L}\left(\mathbb{K}^{n}, \mathbb{K}\right)$. These equalities follow directly from definitions of $\nu, \kappa, \gamma$ and show that these functions coincide on $\mathcal{L}\left(\mathbb{K}^{n}, \mathbb{K}\right)$ and, from Lemma 1.3.11 one has that $\zeta$ is equivalent to these three functions. Moreover, they are norms on $\mathcal{L}\left(\mathbb{K}^{n}, \mathbb{K}\right)$.

We have seen that Lemma 1.3.4, Lemma 1.3.6 and Lemma 1.3.8 give relations among Rabier, Kuo, Gaffney and Jelonek functions. These relations can be described in the following way:

Lemma 1.3.11 ([24, Propositions 2.3 and 2.4]; [29, Proposition 2.6]). Let $\beta_{1}, \beta_{2}$ be functions such that $\beta_{i} \geq 0$, for $i=1,2$. The notation $\beta_{1} \sim \beta_{2}$ means that there exist positive constants $c_{1}, c_{2}$ such that $c_{1} \beta_{1} \leq \beta_{2} \leq c_{2} \beta_{1}$. The relation " $\sim$ " is an equivalence relation and we have

$$
\kappa \sim \nu \sim \gamma \sim \zeta
$$

\footnotetext{
${ }^{1}$ we consider only indices $j$ and $J$ such that $\left\|M_{J, j}(A)\right\| \neq 0$; if all $\left\|M_{J, j}(A)\right\|$ are zero then we set $\zeta(A)=0$.
} 
Note that, from the above lemma, we have: $\nu(A)=0 \Leftrightarrow \kappa(A)=0 \Leftrightarrow \gamma(A)=0 \Leftrightarrow \zeta(A)=$ 0 .

\subsection{Relative Functions}

In some parts of our work, we consider mappings $f: X \rightarrow \mathbb{K}^{p}$, where $X \subset \mathbb{K}^{n}$ is a smooth variety. In this case, we shall need to consider linear mappings defined on a linear subspace of $\mathbb{K}^{n}$.

Thus, we present in this section the functions $\nu, \kappa, \gamma$ and $\zeta$ defined on the space $\mathcal{L}\left(H, \mathbb{K}^{p}\right)$, where $H$ is a proper linear subspace of $\mathbb{K}^{n}$. To distinguish these functions from the functions presented in $\S 1.3$, we call the functions $\nu, \kappa$ and $\gamma$ defined on $\mathcal{L}\left(H, \mathbb{K}^{p}\right)$ respectively by relative Rabier function, relative Kuo function, relative Gaffney function and relative Jelonek function. We begin with:

\section{Relative Rabier Function}

Firstly, we remark that the Rabier Function was defined on $\mathcal{L}(V, W)$, where $V, W$ are any normed finite dimensional vector spaces over $\mathbb{R}$ or $\mathbb{C}$.

Therefore, for any proper linear subspace $H$ of $V$ and any linear mapping $A \in \mathcal{L}(V, W)$, the Rabier function is well-defined on the restriction $A_{\mid H}$ in the following way: we regard the restriction $A_{\mid H}$ as an element of $\mathcal{L}(H, W)$ and, since the function $\nu$ is defined on $\mathcal{L}(H, W)$ (see Definition 1.3.1), we can consider $\nu\left(A_{\mid H}\right)$. However, to establish the notations, we have:

Definition 1.4.1. Let $A \in \mathcal{L}(V, W)$ and let $H$ be a linear subspace of $V$. Denote by $A_{\mid H}$ the restriction of $A$ to $H$. We set the relative Rabier function of $A_{\mid H}$ as:

$$
\nu(A, H):=\nu\left(A_{\mid H}\right)
$$

where $\nu\left(A_{\mid H}\right)$ is defined as in Definition 1.3.1 (i.e., $\nu\left(A_{\mid H}\right)=\inf \left\{\left\|\left(A_{\mid H}\right)^{*}(\varphi)\right\| ; \varphi \in\right.$ $W^{*}$ and $\left.\left.\|\varphi\|=1\right\}\right)$.

Given a vector $w=\left(w_{1}, \ldots, w_{m}\right) \in \mathbb{K}^{m}$, we denote by $[w]$ the line matrix associated to the vector $w$, i.e., $[w]=\left[\begin{array}{lll}w_{1} & \ldots & w_{m}\end{array}\right]$. If $H$ is a linear subspace of $\mathbb{K}^{n}$ then we set $H^{\perp}:=\left\{w \in \mathbb{K}^{n} \mid\langle w, v\rangle=0, \forall v \in H\right\}$. Under these notations, one has:

Lemma 1.4.2. Let $A \in \mathcal{L}\left(\mathbb{K}^{n}, \mathbb{K}^{p}\right)$, and let $H$ be a linear subspace of $\mathbb{K}^{n}$. We denote by $[\mathbf{a}]$ the matrix of $A$ and we set

$$
\nu_{1}(A, H):=\inf \left\{\|[u][\mathbf{a}]+[w]\| ; w \in H^{\perp}, u \in \mathbb{K}^{p} \text { and }\|u\|=1\right\} .
$$

Then there exist positive constants $c_{1}$ and $c_{2}$ such that $c_{1} \nu_{1}(A, H) \leq \nu(A, H) \leq c_{2} \nu_{1}(A, H)$. In other words, $\nu_{1}$ and $\nu$ are equivalent.

Proof. The proof follows directly from Lemma 1.2.5 and Definition 1.4.1. 


\section{Relative Kuo Function}

Definition 1.4.3 (Jelonek [25, Proposition 2.3]). Let $A=\left(A_{1}, \ldots, A_{p}\right) \in \mathcal{L}\left(\mathbb{K}^{n}, \mathbb{K}^{p}\right)$ and let $H \subset \mathbb{K}^{n}$ be a linear subspace defined by a system of independent linear equations $\sum_{j=1}^{n} b_{k j} x_{j}=0$, for $k=1, \ldots, r$ (i.e., $H$ is defined by $r$ equations). One denotes $B_{k}:=\left(b_{k 1}, \ldots, b_{k n}\right)$, for $k=1, \ldots, r$.

The relative Kuo function of $A_{\mid H}$ is defined as follows:

$$
\kappa(A, H):=\min _{1 \leq i \leq p}\left\{\operatorname{dist}\left(A_{i} ;\left\langle\left(A_{l}\right)_{l \neq i},\left(B_{k}\right)_{k=1, \ldots, r}\right\rangle\right)\right\}
$$

where $\left\langle\left(A_{l}\right)_{l \neq i},\left(B_{k}\right)_{k=1, \ldots, r}\right\rangle$ denotes the subspace generated by the vectors $\left\{A_{l} \mid l \neq i\right\}$ and by the vectors $\left\{B_{1}, \ldots, B_{r}\right\}$.

\section{Relative Gaffney Function}

Definition 1.4.4 ([25, Definition 2.3]). Let $A \in \mathcal{L}\left(\mathbb{K}^{n}, \mathbb{K}^{p}\right)$ and let $H \subset \mathbb{K}^{n}$ be a linear subspace given by a system of independent linear equations $\sum_{j=1}^{n} b_{i j} x_{j}=0$, for $i=1, \ldots, r$, where $\operatorname{dim} H=n-r>p$. Let $[\mathbf{a}]=\left(a_{i j}\right)$ be the matrix of $A$ and $[\mathbf{c}]=\left(c_{i j}\right)$ the $(p+r) \times n$ (i.e., $p+r$ rows and $n$ columns) matrix, where $c_{i j}=a_{i j}$, if $1 \leq i \leq p$ and $c_{i j}=b_{(i-p) j}$, if $p<i \leq p+r$.

Let $I=\left(i_{1}, \ldots, i_{p+r}\right)$ and $J=\left(j_{1}, \ldots, j_{p+r-1}\right)$ be multi-indices with $i_{1}<\ldots<i_{p+r}$ and $j_{1}<\ldots<j_{p+r-1}$. One denotes by $M_{I}\left(A_{\mid H}\right)$ the maximal minor of $[\mathbf{c}]$ formed from the columns indexed by $I$. One denotes by $M_{J, j}\left(A_{\mid H}\right)$ the minor of $[\mathbf{c}]$ of size $(p+r-1)$ using the columns indexed by $J$, and all the rows of the matrix $[\mathbf{c}]$ except for the $j$ th row.

With these notations, we define the relative Gaffney function of $A_{\mid H}$ as follows:

$$
\gamma(A, H):=\frac{\left(\sum_{I}\left\|M_{I}\left(A_{\mid H}\right)\right\|^{2}\right)^{1 / 2}}{\left(\sum_{J, 1 \leq j \leq p}\left\|M_{J, j}\left(A_{\mid H}\right)\right\|^{2}\right)^{1 / 2}} .
$$

If $\sum_{J, 1 \leq j \leq p}\left\|M_{J, j}\left(A_{\mid H}\right)\right\|^{2}=0$, we set $\gamma(A, H)=0$.

\section{Relative Jelonek Function}

Definition 1.4.5 ([25, Definition 2.5]). Let $A \in \mathcal{L}\left(\mathbb{K}^{n}, \mathbb{K}^{p}\right)$ and let $H \subset \mathbb{K}^{n}$ be a linear subspace given by a system of independent linear equations $\sum_{j=1}^{n} b_{i j} x_{j}=0$, for $i=1, \ldots, r$, where $\operatorname{dim} H=n-r>p$. With the notations of Definition 1.4.4, the relative Jelonek function is defined as follows:

$$
\zeta(A, H):=\max _{I}\left\{\min _{J \subset I, 1 \leq j \leq p} \frac{\left(\left\|M_{I}\left(A_{\mid H}\right)\right\|^{2}\right)^{1 / 2}}{\left(\left\|M_{J, j}\left(A_{\mid H}\right)\right\|^{2}\right)^{1 / 2}}\right\},
$$


where we consider only indices $j$ and multi-indices $J$ such that $\left\|M_{J, j}\left(A_{\mid H}\right)\right\| \neq 0$; if all $\left\|M_{J, j}\left(A_{\mid H}\right)\right\|$ are zero then we set $\zeta(A, H)=0$.

From [25, Proposition 2.5] and Lemma 1.4.2, one has:

Lemma 1.4.6. Let $A \in \mathcal{L}\left(\mathbb{K}^{n}, \mathbb{K}^{p}\right)$ and let $H \subset \mathbb{K}^{n}$ be a linear subspace. We have the following relations:

$$
\nu(A, H) \sim \kappa(A, H) \sim \zeta(A, H) \sim \gamma(A, H) \sim \nu_{1}(A, H) .
$$




\title{
Regularity conditions at infinity
}

\author{
Contents \\ 2.1 Introduction ............................ 18 \\ 2.2 Polynomial functions $\ldots \ldots \ldots \ldots \ldots$ \\ 2.3 Affine regularity conditions at infinity for mappings . . . . . . 24 \\ 2.3.1 Fibration Theorem .................. 25

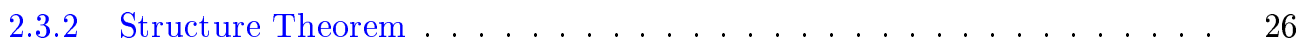 \\ 2.3.3 Asymptotic Morse-Sard type theorem . . . . . . . . . . . . 26

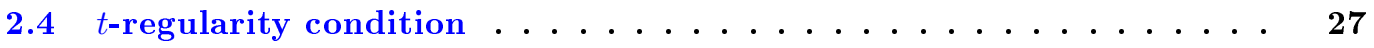 \\ 2.4.1 Localized version of some regularity conditions . . . . . . . . . . 28

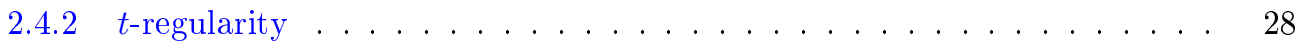 \\ 2.4.3 Regularity conditions and the $t$-regularity . . . . . . . . . . 30

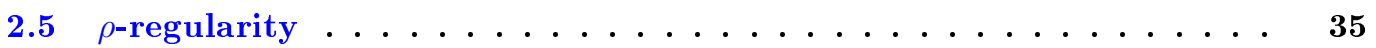

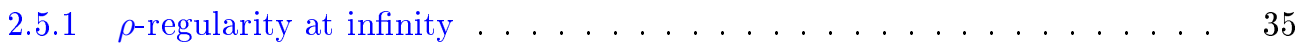 \\ 2.5.2 t-regularity and $\rho_{E}$-regularity . . . . . . . . . . . . 36
}

\section{Brief Resume}

In this chapter, we consider a geometric regularity condition, called $t$-regularity (Definition 2.4.6), in order to study the bifurcation values of semi-algebraic mappings $f: \mathbb{R}^{n} \rightarrow \mathbb{R}^{p}$ and complex polynomial mappings $f: \mathbb{C}^{n} \rightarrow \mathbb{C}^{p}$.

In Theorem 2.4.8, we show that $t$-regularity condition is equivalent to regularity conditions which have been used to approximate $B(f)$ in the works of Rabier [48], Gaffney [18], Jelonek [24, 25], Kurdyka, Orro and Simon [29].

In $\S 2.5$, we consider another regularity condition, called $\rho_{E}$-regularity, and we show that the $\rho_{E}$-regularity refines the above conditions (Propostion 2.5.4 and Proposition 2.5.5).

The main results of $\S 2.4$ and $\S 2.5$ have been published in [11]. 


\subsection{Introduction}

Let $f: \mathbb{K}^{n} \rightarrow \mathbb{K}^{p}$ be a differentiable mapping of class $C^{2}$, where $n \geq p$ and $\mathbb{K}=\mathbb{R}$ or $\mathbb{C}$. We have defined the bifurcation set of $f$, denoted by $B(f)$, as the smallest subset of $\mathbb{K}^{p}$ such that $f$ is a locally trivial fibration over $\mathbb{K}^{p} \backslash B(f)$ (Definition 1 ).

The elements of $B(f)$ may come not only from the critical values but also from the asymptotic behaviour of the fibres. One can see this phenomenon in the next:

EXAmple 2.1.1 (Broughton [4, page 168]). Consider the polynomial function $f: \mathbb{K}^{2} \rightarrow \mathbb{K}$ defined by $f(x, y)=x(x y+1)$. One has $\nabla f(x, y)=\left(2 x y+1, x^{2}\right)$, hence $f$ is a submersion, i.e. Sing $f=\emptyset$. From the definition of $f$, we have the following equalities:

$$
\begin{gathered}
f^{-1}(0)=\{(x, y) \mid x=0 \text { or } x y=-1\} \cong \mathbb{K} \sqcup(\mathbb{K} \backslash\{0\}), \\
f^{-1}(\epsilon)=\left\{(x, y) \mid x \neq 0 \text { and } y=(\epsilon-x) / x^{2}\right\} \cong \mathbb{K} \backslash\{0\}, \text { for any } \epsilon \neq 0,
\end{gathered}
$$

where the notation $S_{1} \cong S_{2}$ means that $S_{1}$ is homeomorphic to $S_{2}$. From Remark 1, and since $f^{-1}(0)$ is not homeomorphic to $f^{-1}(\epsilon)$, for any $\epsilon \neq 0$, we get that $0 \in B(f) \backslash(B(f) \cap f(\operatorname{Sing} f))$.

In other words, this example shows that regular fibres of $f$ may contribute to the set $B(f)$, i.e., the set $B(f) \backslash(B(f) \cap f(\operatorname{Sing} f))$ may be non-empty. It has therefore been imagined various ways to describe the sets $B(f)$ and $B(f) \backslash(B(f) \cap f(\operatorname{Sing} f))$.

An approach to control the points in $B(f) \backslash(B(f) \cap f(\operatorname{Sing} f)$ ) (or to control the asymptotic behaviour of the fibres) is through appropriate regularity conditions at infinity. Under a regularity condition at infinity, we can define a subset $\mathcal{N} \mathcal{R}_{\infty}(f)$ in $\mathbb{K}^{p}$ of non-regular values at infinity in order to obtain a fibration for $f$ outside the union $\left(\mathcal{N} \mathcal{R}_{\infty}(f) \cup f(\operatorname{Sing} f)\right)$. Therefore, regularity conditions at infinity yield sets $\mathcal{N} \mathcal{R}_{\infty}(f)$ which contains the set $B(f) \backslash$ $(B(f) \cap f(\operatorname{Sing} f))$ (see also Example 4.2.10).

In this case, showing that a set $\mathcal{N} \mathcal{R}_{\infty}(f)$ of non-regular values at infinity contains $B(f) \backslash$ $(B(f) \cap f(\operatorname{Sing} f))$, means to prove that $f$ is a fibration on $\mathbb{K}^{p} \backslash\left(f(\operatorname{Sing} f) \cup \mathcal{N} \mathcal{R}_{\infty}(f)\right)$, i.e, one proves a "fibration theorem" for the regularity condition at infinity that yields $\mathcal{N} \mathcal{R}_{\infty}(f)$.

Moreover, it is natural to ask about the structure of the set of non-regular values at infinity $\mathcal{N} \mathcal{R}_{\infty}(f)$. In other words, we ask if $\mathcal{N R}_{\infty}(f)$ has properties which imply a good approximation for $B(f) \backslash(B(f) \cap f(\operatorname{Sin} g f))$. Equivalently, we expect that the regularity condition defining $\mathcal{N} \mathcal{R}_{\infty}(f)$ is not too strong (compare with the Example 2.2.8). In this way, we are interested to obtain sets $\mathcal{N} \mathcal{R}_{\infty}(f)$ with properties that imply that the set $\left(\mathcal{N} \mathcal{R}_{\infty}(f) \cup f(\operatorname{Sing} f)\right)$ is a closed set with measure zero, and consequently, it follows that $\mathbb{K}^{p} \backslash\left(\mathcal{N} \mathcal{R}_{\infty}(f) \cup f(\operatorname{Sing} f)\right)$ is open dense on $\mathbb{K}^{p}$. Thus, showing that some set $\mathcal{N R}_{\infty}(f)$ has these properties, means to prove a "structure theorem" for the regularity condition at infinity that yields $\mathcal{N} \mathcal{R}_{\infty}(f)$.

In each of the above cases, showing that a regularity condition at infinity yields a set $\mathcal{N} \mathcal{R}_{\infty}(f)$ of non-regular values that gives a fibration theorem and a structure theorem, means to prove an asymptotic Morse-Sard type theorem together with a fibration theorem to non- 
proper mappings $f: \mathbb{K}^{n} \rightarrow \mathbb{K}^{p}$.

In this chapter, we focus on regularity conditions at infinity for $C^{1}$ semi-algebraic mappings $f: \mathbb{R}^{n} \rightarrow \mathbb{R}^{p}$ and complex polynomial mappings $f: \mathbb{C}^{n} \rightarrow \mathbb{C}^{p}$. These regularity conditions at infinity will be presented in two main contexts:

\section{i. Global Context:}

Given a $C^{1}$ mapping $f: \mathbb{K}^{n} \rightarrow \mathbb{K}^{p}, \mathbb{K}=\mathbb{R}, \mathbb{C}$, we consider special functions " $\beta$ " on $\mathcal{L}\left(\mathbb{K}^{n}, \mathbb{K}^{p}\right)$ and special regularity conditions, which depend of $f$ and $\beta$. The key fact is that these regularity conditions are required on the affine space $\mathbb{K}^{n} \backslash \mathrm{B}_{\mathrm{R}}(0)$, where $\mathrm{R}$ is an integer big enough and $B_{R}(0)$ denotes the euclidean ball of radius $R$ centered at 0 . Therefore, in this way we work only on the affine space $\mathbb{K}^{n}$. This context is global in the sense that we need to verify a condition on the space $\mathbb{K}^{n}$. See $\S 2.3$ and $\S 2.5$.

ii. Local Context:

Given a semi-algebraic mapping $f: \mathbb{R}^{n} \rightarrow \mathbb{R}^{p}$ or a complex polynomial mapping $f: \mathbb{C}^{n} \rightarrow$ $\mathbb{C}^{p}$, we consider the closure of the graph of $f$ in the space $\mathbb{P}^{n} \times \mathbb{K}^{p}$ (via the canonical embedding of $\mathbb{K}^{n}$ in the projective space $\mathbb{P}^{n}$ ) and we fix a point $z_{0}$ on the boundary of the graph of $f$ in $\mathbb{P}^{n} \times \mathbb{K}^{p}$. Under these assumptions, the regularity conditions are formulated on a small enough neighbourhood of $z_{0}$ in $\mathbb{P}^{n} \times \mathbb{K}^{p}$. Thus, we work locally on the point $z_{0}$ to obtain some results for $f$. See $\S 2.4$ and $\S 2.5$.

The aims of the first two sections, section 2.2 and section 2.3, are to present results from the literature that are strongly related with our work. In section 2.2 , we focus on polynomial functions and in section 2.3, we focus on mappings.

In sections 2.4 and 2.5, we consider two regularity conditions at infinity for semi-algebraic mappings $f: \mathbb{R}^{n} \rightarrow \mathbb{R}^{p}$ and for complex polynomial mappings $f: \mathbb{C}^{n} \rightarrow \mathbb{C}^{p}$. We discuss some properties of these conditions and present some of our results.

The chapter is structured as follows:

In section 2.2, we present some well-known facts about the bifurcation set of polynomial functions $f: \mathbb{K}^{n} \rightarrow \mathbb{K}$ and discuss some regularity conditions at infinity which have been used to approximate the elements of $B(f) \backslash(B(f) \cap f(\operatorname{Sing} f))$. These regularity conditions include the $t$-regularity, the $\rho_{E}$-regularity and the Malgrange condition.

We explain the relation of these last three regularity conditions with our work and with the conditions considered in 2.3. Thus, we will see that the conditions of $\S 2.3, \S 2.4$ and $\S 2.5$, in some sense, are extensions of those three regularity conditions to the context of mappings, see also Remark 2.3.4, Remark 2.4.10 and §2.5.

In section 2.3, we discuss some important results from the works of Rabier [48], Gaffney [18], Jelonek [24, 25] and Kurdyka, Orro, Simon [29]. We present four regularity conditions at infinity: Rabier condition, Kuo-KOS condition, Gaffney condition and Jelonek condition; we discuss some of its properties and relations obtained in [18, 24, 25, 29, 48]. 
We present that these conditions give a fibration theorem (§2.3.1) and a structure theorem (§2.3.2) for non-proper mappings.

Also, we explain some relations of $[18,24,25,29,48]$ and that the theorems above mentioned give an asymptotic Morse-Sard type theorem for semi-algebraic mappings $f: \mathbb{R}^{n} \rightarrow \mathbb{R}^{p}$ and to complex polynomial mappings $f: \mathbb{C}^{n} \rightarrow \mathbb{C}^{p}$.

In section 2.4, we extend to $C^{1}$ semi-algebraic mapping $f: \mathbb{R}^{n} \rightarrow \mathbb{R}^{p}$ and to complex polynomial mappings $f: \mathbb{C}^{n} \rightarrow \mathbb{C}^{p}$ the notion of $t$-regularity. We show some properties of this condition. We reformulate Rabier, Kuo-KOS, Gaffney and Jelonek conditions in localized versions. This new interpretation allows one to show the equivalence between $t$-regularity with these four regularity conditions for semi-algebraic mappings and for complex polynomial mappings (Theorem 2.4.8). We explain how these results generalize some results of [43, 49].

In section 2.5, we define the $\rho_{E}$-regularity condition for semi-algebraic mappings and for complex polynomial mappings. We discuss some properties of this condition and we show that $t$-regularity implies $\rho_{E}$-regularity, and consequently from $\S 2.4$, we obtain that Rabier, Kuo-KOS, Gaffney and Jelonek conditions imply $\rho_{E}$-regularity.

\subsection{Polynomial functions}

Let $f: \mathbb{K}^{n} \rightarrow \mathbb{K}$ be a polynomial function, $\mathbb{K}=\mathbb{R}$ or $\mathbb{C}$. We start with some well-known facts about the bifurcation set of polynomial functions.

\section{Classical results}

We start with:

Theorem 2.2.1. Let $f: \mathbb{K}^{n} \rightarrow \mathbb{K}$ be a polynomial function. Then $B(f)$ is a finite set.

It is very surprising that for the class of polynomial functions (consequently for more general classes of functions), "there is not yet a complete characterization of the set $B(f) \backslash$ $(B(f) \cap f(\operatorname{Sing} f))$ and, consequently, of the set $B(f)$ ".

A complete characterization of $B(f)$ is avaliable only in the case $n=2$, i.e., only for polynomial functions $f: \mathbb{K}^{2} \rightarrow \mathbb{K}$. For $\mathbb{K}=\mathbb{C}$, we have the following characterization:

Theorem 2.2.2 (Suzuki [50]; Hà and Lê [22, page 25]). Let $f: \mathbb{C}^{2} \rightarrow \mathbb{C}$ be a polynomial function. We have $t_{0} \notin B(f)$ if and only if $t_{0}$ is a regular value of $f$ and the Euler characteristic of $f^{-1}\left(t_{0}\right)$ equals to that of the general fibre of $f$.

From Theorem 2.2.1 it follows that $B(f)$ is a finite set and consequently $\mathbb{C} \backslash B(f)$ is a dense open connected set. For any $t$ in this last set, $f^{-1}(t)$ is called a generic fibre of $f$. Thus, 
the fibres of $f$ on a dense open connected set are homeomorphic and, consequently, the Euler characteristic of the fibres of $f$ on this set are the same. Moreover, the above theorem says that, to decide whether a regular value $t_{0}$ of $f$ is a bifurcation value or not, we just need to compute the Euler characteristic of $f^{-1}\left(t_{0}\right)$ and the Euler characteristic of the generic fibre of $f$. The following example illustrates the above theorem:

Example 2.2.3. Consider $f: \mathbb{C}^{2} \rightarrow \mathbb{C}, f(x, y)=x(x y+1)$. We have seen in Example 2.1.1 that $f$ is a submersion, $f^{-1}(0)$ is homeomorphic to $\mathbb{C} \sqcup(\mathbb{C} \backslash\{0\})$ and, for $\epsilon \neq 0, f^{-1}(\epsilon)$ is homeomorphic to $(\mathbb{C} \backslash\{0\})$. This implies $\chi\left(f^{-1}(0)\right)=1$ and $\chi\left(f^{-1}(\epsilon)\right)=0$, where the notation $\chi(S)$ means the Euler characteristic of $S$. Therefore, from Theorem 2.2.2, we conclude the equality $B(f)=\{0\}$.

For $\mathbb{K}=\mathbb{R}$ and $n=2$, one has the following characterization of $B(f)$ :

Theorem 2.2.4 (Tibăr and Zaharia [57, Theorem 2.5]). Let $f: \mathbb{R}^{2} \rightarrow \mathbb{R}$ be a polynomial mapping and let $t_{0}$ be a regular value of $f$. Then the following two conditions are equivalent:

i) $t_{0} \notin B(f)$.

ii) The Euler characteristic $\chi\left(f^{-1}(t)\right)$ is constant for $t$ close enough to $t_{0}$ and there is no connected component of $f^{-1}(t)$ which vanishes at infinity when $t$ tends to $t_{0}$.

REMARK 2.2.5. The above theorem can be formulated in more general settings. In fact, Tibăr and Zaharia [57, Theorem 2.5] present three different equivalent conditions, which describe the set $B(f) \backslash(B(f) \cap f(\operatorname{Sing} f))$ for a polynomial function $f: \mathbb{R}^{2} \rightarrow \mathbb{R}$.

Under additional hypothesis, we can obtain a complete characterization of the bifurcation values for some special classes of polynomial functions. For instance, S. A. Broughton [4, 5] considered the following class:

Definition 2.2.6 (Broughton [4, Definition 1],[5, Definition 3.1]). We say that a polynomial function $f: \mathbb{C}^{n} \rightarrow \mathbb{C}$ is a tame polynomial if there is a compact neighbourhood $U$ of the critical points of $f$ such that $\nabla f(x)$ is bounded away from 0 on $\mathbb{C}^{n} \backslash U$.

For the above class, one has the following characterization of $B(f)$ :

Proposition 2.2.7 (Broughton [4, Proposition 2]). If $f: \mathbb{C}^{n} \rightarrow \mathbb{C}$ is a tame polynomial then $B(f)=f(\operatorname{Sing} f)$.

Let $f: \mathbb{K}^{n} \rightarrow \mathbb{K}$ be a polynomial function. From Definition 2.2.6, we can define the following set:

$$
T_{\infty}(f):=\left\{t_{0} \in \mathbb{K} \mid \exists\left\{x_{j}\right\}_{j \in \mathbb{N}} \subset \mathbb{K}^{n}, \lim _{j \rightarrow \infty}\left\|x_{j}\right\|=\infty, \lim _{j \rightarrow \infty}\left(f\left(x_{j}\right),\left\|\nabla f\left(x_{j}\right)\right\|\right)=\left(t_{0}, 0\right)\right\},
$$


and, directly from Definition 2.2.6, we have that $f$ is tame ${ }^{1}$ if and only if $T_{\infty}(f)=\emptyset$. Moreover, from the proof of Broughton [4, Proposition 2] we get $B(f) \subset T_{\infty}(f) \cup f(\operatorname{Sing} f)$. However, a problem is that the tame condition is not a generic condition. Sometimes, it is a strong condition as the next example shows:

EXAmple 2.2.8 (Parusiński [43, Example 1.11]). Consider $f: \mathbb{K}^{3} \rightarrow \mathbb{K}$ defined by $f(x, y, z)=$ $x+x^{2} y+x^{4} y z$. Then $T_{\infty}(f)=\mathbb{K}$.

Other regularity conditions for polynomial functions have been considered in the literature, which improve considerably the tame condition. These conditions generate interesting classes of polynomials, see for instance Parusiński [42, Definition 1.1 and Theorem 1.4], Siersma and Tibăr [49, Definition 2.4] and Tibăr [56, Section 2.2]. In particular, Némethi [38, 39] defined the class of quasi-tame polynomial functions and Némethi and Zaharia [40, page 686] the class of M-tame polynomial functions (for M-tame, see paragraph before Definition 2.2.11). For these classes of polynomial functions, we have results like Proposition 2.2.7 in the following context:

Proposition 2.2.9. If $f: \mathbb{C}^{n} \rightarrow \mathbb{C}$ is a quasi-tame or $M$-tame polynomial function then $B(f)=f(\operatorname{Sing} f)$.

The proof of the above result can be found in the works of Némethi [39, Theorem 1.13] for the class of quasi-tame and Némethi and Zaharia [40, Theorem 1 and page 686] for the class of $M$-tame.

Malgrange Condition, $t$-regularity and $\rho_{E}$-regularity

We begin with the definition of the Malgrange condition and of the $\rho_{E^{-}}$regularity. Following Pham [47, page 14], we set:

Definition 2.2.10 (Malgrange Condition). Let $f: \mathbb{K}^{n} \rightarrow \mathbb{K}$ be a polynomial function, $\mathbb{K}=\mathbb{R}$ or $\mathbb{C}$. We say that a point $t_{0} \in \mathbb{K}$ satisfies the Malgrange condition if for any sequence $\left\{x_{j}\right\}_{j \in \mathbb{N}} \subset \mathbb{K}^{n}$ with the properties $\lim _{j \rightarrow \infty}\left\|x_{j}\right\|=\infty$ and $\lim _{j \rightarrow \infty} f\left(x_{j}\right)=t_{0}$, the expression $\left\|x_{j}\right\|\left\|\nabla f\left(x_{j}\right)\right\|$ does not converge to zero, as $j$ tends to infinity.

The next condition is a condition of transversality of $f$ to the Euclidean distance function $\rho_{E}$. The transversality of the fibres of $f$ to the levels of the Euclidean distance is a "Milnortype" condition. This type of condition has been used by Milnor [34] in the local study of singular functions. For complex polynomial functions, transversality to big spheres was used by Broughton [5, page 229] and later by Némethi and Zaharia [40], where a polynomial function $f: \mathbb{K}^{n} \rightarrow \mathbb{K}$ was called M-tame if the transversality to big spheres is satisfied for any fibre of $f$ ([40, page 686]). The use of $\rho_{E}$-regularity for polynomial functions $f: \mathbb{K}^{n} \rightarrow \mathbb{K}$ can

\footnotetext{
${ }^{1}$ we define a tame polynomial $f: \mathbb{K}^{n} \rightarrow \mathbb{K}$ changing $\mathbb{C}$ by $\mathbb{K}$ in Definition 2.2.6.
} 
be seen also in Păunescu and Zaharia [45, page 270]), Parusiński [42, page 381], Siersma and Tibăr [49, page 781] and Tibăr [54, page 93], [55, §2], [56, §1.2].

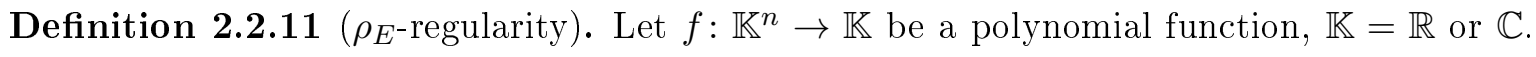
Consider $\mathcal{M}(f):=\left\{x \in \mathbb{K}^{n} \mid \exists \lambda \in \mathbb{K}\right.$ such that $\left.\nabla f(x)=\lambda x\right\}$. We say that a point $t_{0} \in \mathbb{K}$ is $\rho_{E}$-regular if there exists no sequence $\left\{x_{j}\right\}_{j \in \mathbb{N}} \subset \mathcal{M}(f)$ such that $\lim _{j \rightarrow \infty}\left\|x_{j}\right\|=\infty$ and $\lim _{j \rightarrow \infty} f\left(x_{j}\right)=t_{0}$.

REMARK 2.2.12. Note that the set $\mathcal{M}(f)$ is just the union of the critical points of $f$ and the points in $\mathbb{K}^{n}$ where the transversality of spheres with the fibres of $f$ is not satisfied.

The above conditions generate the set of points in $\mathbb{K}$ that does not satisfy the Malgrange condition and the set of points that does not satisfy the $\rho_{E}$-regularity, which we denote respectively by $\mathrm{MC}_{\infty}(f)$ and $S(f)$. We have respectively the following characterizations for these sets:

$$
\begin{gathered}
\mathrm{MC}_{\infty}(f)=\left\{t_{0} \in \mathbb{K} \mid \exists\left\{x_{j}\right\}_{j \in \mathbb{N}} \subset \mathbb{K}^{n}, \lim _{j \rightarrow \infty}\left\|x_{j}\right\|=\infty,\right. \\
\left.\lim _{j \rightarrow \infty} f\left(x_{j}\right)=t_{0} \text { and } \lim _{j \rightarrow \infty}\left\|x_{j}\right\|\left\|\nabla f\left(x_{j}\right)\right\|=0\right\}, \text { and } \\
S(f)=\left\{t_{0} \in \mathbb{K} \mid \exists\left\{x_{j}\right\}_{j \in \mathbb{N}} \subset \mathcal{M}(f), \lim _{j \rightarrow \infty}\|x\|=\infty \text { and } \lim _{j \rightarrow \infty} f\left(x_{j}\right)=t_{0}\right\},
\end{gathered}
$$

where $\mathcal{M}(f)$ is as in Definition 2.2.11.

An important fact for polynomial functions (i.e. $\left.f: \mathbb{K}^{n} \rightarrow \mathbb{K}\right)$ is that $B(f) \subset(f(\operatorname{Sing} f) \cup$ $S(f))$ and $B(f) \subset\left(f(\operatorname{Sing} f) \cup \mathrm{MC}_{\infty}(f)\right)$. Moreover, in contrast to the tame condition (see Example 2.2.8), the sets $(f(\operatorname{Sing} f) \cup S(f))$ and $\left(f(\operatorname{Sing} f) \cup \mathrm{MC}_{\infty}(f)\right)$ represent a good approximation of $B(f)$ by the fact that they are finite sets (see Remark 2.2.13).

Another regularity condition, which will be defined for semi-algebraic mappings $f: \mathbb{R}^{n} \rightarrow$ $\mathbb{R}^{p}$ and for complex polynomial mappings $f: \mathbb{C}^{n} \rightarrow \mathbb{C}^{p}$ in section 2.4, is the t-regularity (also called $t$-equisingularity) ${ }^{2}$. This regularity condition is a type of non-characteristic condition at infinity (a geometric condition) and it was considered by Siersma and Tibăr [49, §5] in the case of polynomial functions $f: \mathbb{K}^{n} \rightarrow \mathbb{K}$ (see also Parusiński [42, 43], Tibăr [53, 54]) . Remarkably, Siersma and Tibăr [49, page 780] proved that t-regularity implies Malgrange condition and, Parusiński [43, §1] showed that Malgrange condition implies t-regularity (the proof of this equivalence can also be seen in Parusiński [44, Theorem 3.1] and Tibăr [56, Proposition 1.3.2]).

Therefore, for polynomial functions $f: \mathbb{K}^{n} \rightarrow \mathbb{K}$, we have the equivalence $t$-regularity iff Malgrange condition. Moreover, $t$-regularity implies $\rho_{E}$-regularity (Tibăr [55, Proposition 2.11]) and, consequently, the inclusion of the sets $S(f) \subset \mathrm{MC}_{\infty}(f)$ holds. We shall see that

\footnotetext{
${ }^{2}$ the $t$-regularity will be presented in details in section 2.4 for mappings (see also 2.5.2 and 4.2.2).

3 see also Remark 2.2.14.
} 
some results of sections 2.4 and 2.5, in some sense, are generalizations of these results in the setting of semi-algebraic mappings and complex polynomial mappings.

The next figure presents the above results in a condensed manner:

Figure 2.1: Malgrange condition, $t$-regularity and $\rho_{E}$-regularity.

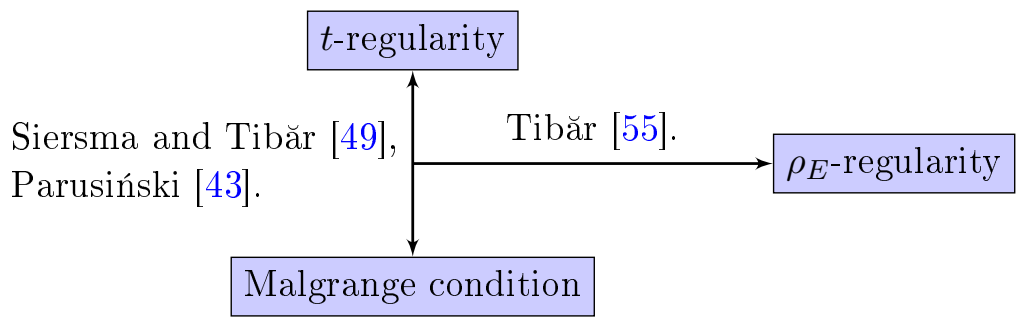

REMARK 2.2.13. The fact that Malgrange condition and $\rho_{E}$-regularity condition imply fibration theorems (i.e., $B(f) \subset(f(\operatorname{Sing} f) \cup S(f))$ and $\left.B(f) \subset\left(f(\operatorname{Sing} f) \cup \mathrm{MC}_{\infty}(f)\right)\right)$ follows from standard arguments, see for instance Milnor [34] (arguments in the local case), and, in the global case, see for instance Broughton [5, page 229], Némethi and Zaharia [40, Theorem 1], Parusiński [42, Lemma 1.2 and Remark 1.3], Siersma and Tibăr [49, proof of Proposition 5.5], etc.... See also Chapter 3 and $\S 1.1$.

The finiteness of $\mathrm{MC}_{\infty}(f)$ follows from Siersma and Tibăr [49, page 779] and from the link between $t$-regularity and Malgrange condition. The finiteness of $S(f)$ follows by the fact that $\mathrm{MC}_{\infty}(f)$ is finite and by the inclusion $S(f) \subset \mathrm{MC}_{\infty}(f)$.

REMARK 2.2.14. Connected to the definition of $t$-regularity, we have the notions of relative conormal and characteristic covectors at infinity. These notions have been used by Siersma and Tibăr [49] (see also Tibăr $[53,55,56]$ ) in the case of polynomial functions $f: \mathbb{K}^{n} \rightarrow \mathbb{K}$.

\subsection{Affine regularity conditions at infinity for mappings}

Let $f: \mathbb{K}^{n} \rightarrow \mathbb{K}^{p}$ be a $C^{1}$ mapping, where $\mathbb{K}=\mathbb{R}, \mathbb{C}$ and $n \geq p$. From the functions defined in $\S 1.3$, we set the following:

Definition 2.3.1. Let $f: \mathbb{K}^{n} \rightarrow \mathbb{K}^{p}$ be a $C^{1}$ mapping, with $n \geq p$. We define:

$$
\begin{aligned}
\mathcal{N}_{\infty}(f):= & \left\{t \in \mathbb{K}^{p} \mid \exists\left\{x_{j}\right\}_{j \in \mathbb{N}} \subset \mathbb{K}^{n}, \lim _{j \rightarrow \infty}\left\|x_{j}\right\|=\infty,\right. \\
& \left.\lim _{j \rightarrow \infty} f\left(x_{j}\right)=t \text { and } \lim _{j \rightarrow \infty}\left\|x_{j}\right\| \beta\left(\operatorname{D} f\left(x_{j}\right)\right)=0\right\},
\end{aligned}
$$

where $\mathrm{D} f(x)$ denotes the Jacobian of $f$ at $x$; and $\beta$ stands for the Rabier function $\nu$ (Definition 1.3.1), the Kuo function $\kappa$ (Definition 1.3.3), the Gaffney function $\gamma$ (Definition 1.3.5), the Jelonek function $\zeta$ (Definition 1.3.7). 
From Lemma 1.3.11 (due to Jelonek [24] and Kurdyka, Orro, Simon [29]), it follows that $\mathcal{N}_{\infty}(f)$ does not depend on the chosen function. In other words, the functions $\nu, \kappa, \gamma, \zeta$ yield the same set $\mathcal{N}_{\infty}(f)$.

Automatically, the set $\mathcal{N}_{\infty}(f)$ give a regularity condition on $f$, in the sense that $t_{0} \notin$ $\mathcal{N}_{\infty}(f)$ means that $t_{0}$ satisfies a regularity condition for the functions $\nu, \kappa, \gamma, \zeta$. More precisely, one has:

Definition 2.3.2. Let $f: \mathbb{K}^{n} \rightarrow \mathbb{K}^{p}$ be a $C^{1}$ mapping. We say that $t_{0}$ satisfies Rabier condition (respectively Kuo-KOS condition ${ }^{4}$, Gaffney condition, Jelonek condition) when we use the function $\nu$ (respectively $\kappa, \gamma, \zeta)$ to generate the set $\mathcal{N}_{\infty}(f)$ and $t_{0} \notin \mathcal{N}_{\infty}(f)$.

From Lemma 1.3.11, it follows that $t_{0}$ satisfies Rabier condition $\Leftrightarrow t_{0}$ satisfies Kuo-KOS condition $\Leftrightarrow t_{0}$ satisfies Gaffney condition $\Leftrightarrow t_{0}$ satisfies Jelonek condition.

REMARK 2.3.3. Originally, the set generated by $\nu$ has been called asymptotic critical values of $f$ and denoted by $K_{\infty}(f)([48$, page 668 and Remark 6.1]).

The next remark explains how these conditions generalize the Malgrange condition (Definition 2.2.10).

Remark 2.3.4. From Remark 1.3.10, we have $\nu(A)=\kappa(A)=\gamma(A)=\zeta(A)=\|A\|$, for any $A \in \mathcal{L}\left(\mathbb{K}^{n}, \mathbb{K}\right)$. These equalities and Definition 2.2.10 imply that the four above conditions are just the Malgrange condition in the setting of polynomial functions $f: \mathbb{K}^{n} \rightarrow \mathbb{K}$. We also remark that the condition generated by $\gamma$ was originally called by Gaffney [18] of generalized Malgrange condition.

From the literature, we present two important results on $\mathcal{N}_{\infty}(f)$ : a fibration theorem and a structure theorem.

\subsubsection{Fibration Theorem}

We have seen in the previous subsection that Rabier condition, Kuo-KOS condition, Gaffney condition and Jelonek condition yield the same set $\mathcal{N}_{\infty}(f)$ (Definition 2.3.1) for a $C^{1}$ mapping $f: \mathbb{K}^{n} \rightarrow \mathbb{K}^{p}$. For this, we present:

Theorem 2.3.5. Let $f: \mathbb{K}^{n} \rightarrow \mathbb{K}^{p}$ be a $C^{2}$ differentiable mapping. Then the restriction:

$$
f_{\mid}: \mathbb{K}^{n} \backslash f^{-1}(\mathcal{N}(f)) \rightarrow \mathbb{K}^{p} \backslash \mathcal{N}(f)
$$

is a locally trivial fibration, where $\mathcal{N}(f):=f(\operatorname{Sing} f) \cup \mathcal{N}_{\infty}(f)$ and $\mathcal{N}_{\infty}(f)$ is defined as in Definition 2.3.1. In particular, $B(f) \subset \mathcal{N}(f)$ and $(B(f) \backslash(B(f) \cap f(\operatorname{Sing} f))) \subset \mathcal{N}_{\infty}(f)$.

\footnotetext{
${ }^{4}$ The use of the Kuo function to study the bifurcation values is due to Kurdyka, Orro and Simon [29], and it is for this reason that we say Kuo-KOS condition, see Lemma 1.3.4.
} 
Rabier, by using the function $\nu$ (introduced by himself), obtained in [48, §4] a proof of Theorem 2.3.5 in the more general setting of maps $f: M \rightarrow N$, where $M, N$ are Finsler Manifolds.

Jelonek [24, Theorem 3.1] gives a different and more direct proof of Theorem 2.3.5 with the Jelonek function for smooth mappings $f: \mathbb{K}^{n} \rightarrow \mathbb{K}^{p}$, where $\mathbb{K}=\mathbb{R}, \mathbb{C}, n \geq p$. We remark that the same proof holds for $C^{2}$ mappings.

In summary, for $C^{2}$ mappings $f: \mathbb{K}^{n} \rightarrow \mathbb{K}^{p}, \mathbb{K}=\mathbb{R}, \mathbb{C}, n \geq p$, since from Lemma 1.3.11 one has the relation of Rabier function with Kuo function (respectively Gaffney function, or Jelonek function), we have that Theorem 2.3.5 with the Kuo function $\kappa$ (respectively the Gaffney function $\gamma$, or the Jelonek function $\zeta$ ) can be deduced from these links and from the work of Rabier [48, §4].

It is interesting to remark that, using the function $\gamma$, Gaffney [18, Theorems 1.7 and 1.8] gives a different proof of Theorem 2.3.5 (without using of Rabier's work) for fair ${ }^{5}$ complex polynomial mappings $f: \mathbb{C}^{n} \rightarrow \mathbb{C}^{p}$.

\subsubsection{Structure Theorem}

In this subsection, we consider a $C^{1}$ semi-algebraic mapping $f: \mathbb{R}^{n} \rightarrow \mathbb{R}^{p}$ and/or a polynomial mapping $f: \mathbb{C}^{n} \rightarrow \mathbb{C}^{p}$. We have the following structure theorem:

Theorem 2.3.6 ([29, Theorem 3.1 and Theorem 4.1]). Let $f: \mathbb{R}^{n} \rightarrow \mathbb{R}^{p}$ be a $C^{1}$ semialgebraic mapping (respectively let $f: \mathbb{C}^{n} \rightarrow \mathbb{C}^{p}$ be a polynomial mapping). Then $\mathcal{N}_{\infty}(f)$ and $\mathcal{N}(f):=\mathcal{N}_{\infty}(f) \cup f(\operatorname{Sing} f)$ are semi-algebraic sets of dimension at most $p-1$ (respectively $\mathcal{N}_{\infty}(f)$ and $\mathcal{N}(f):=\mathcal{N}_{\infty}(f) \cup f(\operatorname{Sing} f)$ are complex algebraic sets of dimension at most $\left.p-1\right)$, where $\mathcal{N}_{\infty}(f)$ is defined as in Definition 2.3.1. Moreover, we have that $\mathcal{N}(f)$ is closed.

Since $\mathcal{N}_{\infty}(f)$ and $\mathcal{N}(f)$ are semi-algebraic sets (complex algebraic sets in the complex case) of dimension at most $p-1$, we have that $\mathcal{N}_{\infty}(f)$ and $\mathcal{N}(f)$ have volume zero. In fact, Kurdyka, Orro and Simon show that $\mathcal{N}_{\infty}(f)$ is semi-algebraic (not difficult) and that the volume (remarkably) of $\mathcal{N}_{\infty}(f)$ is zero, which implies that $\mathcal{N}_{\infty}(f)$ has dimension at most $p-1$.

\subsubsection{Asymptotic Morse-Sard type theorem}

At the beginning of section 2.3, we present four regularity conditions at infinity: the Rabier condition, the Kuo-KOS condition, the Gaffney condition and the Jelonek condition. These regularity conditions yields the same set $\mathcal{N}_{\infty}(f)$ (Definition 2.3.1 and Definition 2.3.2).

\footnotetext{
${ }^{5}$ For definition of fair complex polynomial mapping see 4.2.2.
} 
For a $C^{2}$ mapping $f: \mathbb{K}^{n} \rightarrow \mathbb{K}^{p}$, we set $\mathcal{N}(f):=\mathcal{N}_{\infty}(f) \cup f(\operatorname{Sing} f)$ and we have seen that $f$ is a fibration on $\mathbb{K}^{p} \backslash \mathcal{N}(f)$ (Theorem 2.3.5). In other words, one has a fibration theorem for the regularity conditions defining the set $\mathcal{N}(f)$. Consequently, the inclusion $B(f) \subset \mathcal{N}(f)$ holds.

If we consider a $C^{2}$ semi-algebraic mapping $f: \mathbb{R}^{n} \rightarrow \mathbb{R}^{p}$ or a complex polynomial mapping $f: \mathbb{C}^{n} \rightarrow \mathbb{C}^{p}$, the above four conditions give a structure theorem (Theorem 2.3.6). In particular, this theorem says that $\mathcal{N}(f)$ is closed and has volume zero, which imply that $\mathbb{K}^{p} \backslash \mathcal{N}(f)$ is a dense open set in $\mathbb{K}^{p}$.

Therefore, for a $C^{2}$ semi-algebraic mapping $f: \mathbb{R}^{n} \rightarrow \mathbb{R}^{p}$ or a complex polynomial mapping $f: \mathbb{C}^{n} \rightarrow \mathbb{C}^{p}$, the four above conditions give an asymptotic Morse-Sard type theorem to nonproper mappings $f$.

\section{$2.4 t$-regularity condition}

The central object of this section is the $t$-regularity in the setting of $C^{1}$ semi-algebraic mappings $f: \mathbb{R}^{n} \rightarrow \mathbb{R}^{p}$ and in the setting of complex polynomial mappings $f: \mathbb{C}^{n} \rightarrow \mathbb{C}^{p}$, where $n \geq p$. This regularity condition is a geometric grounded condition that depends of the relative conormal space of an appropriate function $g$ and it is formulated in a localized version on a point of the boundary at infinity of the graph of $f$ in $\mathbb{P}^{n} \times \mathbb{K}^{p}$.

We structure this section as follows:

In §2.4.1, to compare the $t$-regularity condition to the regularity conditions presented in $\S 2.3$, we reformulate the Rabier condition, the Kuo-KOS condition, the Gaffney condition and the Jelonek condition in a localized version on a point of the boundary at infinity of the graph of $f$ in $\mathbb{P}^{n} \times \mathbb{K}^{p}$. In $\S \mathbf{2 . 4 . 2}$, we introduce the main definitions leading to $t$-regularity: the conormal space, the relative conormal space and the space of the characteristic covectors at infinity. We define the $t$-regularity and we give an interpretation for this condition. In §2.4.3, we show that the $t$-regularity condition is equivalent to the regularity conditions presented in $\S 2.3$ (Theorem 2.4.8) and we discuss some consequences.

\section{Notation}

Throughout this section, we use coordinates $\left(x_{1}, \ldots, x_{n}\right)$ for the affine space $\mathbb{K}^{n}$, coordinates $\left[x_{0}: x_{1}: \ldots: x_{n}\right]$ for the projective space $\mathbb{P}^{n}$ and we shall consider the canonical embedding of $\mathbb{K}^{n}$ in $\mathbb{P}^{n}\left(\left(x_{1}, \ldots, x_{n}\right) \mapsto\left[1: x_{1}: \ldots: x_{n}\right]\right)$. We set $\mathbb{H}^{\infty}:=\left\{\left[x_{0}: \ldots: x_{n}\right] \in\right.$ $\left.\mathbb{P}^{n} \mid x_{0}=0\right\}$ the hyperplane at infinity.

Let $f: X \rightarrow \mathbb{K}^{p}$ be a $C^{1}$-mapping, where $X \subset \mathbb{K}^{n}$ is a submanifold. We set $\mathbb{X}:=\overline{\operatorname{graph} f}$ the closure of the graph of $f$ in $\mathbb{P}^{n} \times \mathbb{K}^{p}$ (via the embedding of $\mathbb{K}^{n}$ in $\mathbb{P}^{n}$ ) and we set $\mathbb{X}^{\infty}:=$ $\mathbb{X} \cap\left(\mathbb{H}^{\infty} \times \mathbb{K}^{p}\right)$.

We denote by $\tau: \mathbb{P}^{n} \times \mathbb{K}^{p} \rightarrow \mathbb{K}^{p}$ the second projection, that is $\tau(\mathrm{x}, \mathrm{t})=\mathrm{t}$, for any $(\mathrm{x}, \mathrm{t}) \in$ $\mathbb{P}^{n} \times \mathbb{K}^{p}$. 


\subsubsection{Localized version of some regularity conditions}

We reformulate on a localized version the regularity conditions at infinity presented in Definition 2.3.2. With the above definitions and notations, one has:

Definition 2.4.1. Let $f: \mathbb{K}^{n} \rightarrow \mathbb{K}^{p}$ be a $C^{1}$ semi-algebraic mapping for $\mathbb{K}=\mathbb{R}$ or a complex polynomial mapping for $\mathbb{K}=\mathbb{C}$. We say that $z_{0} \in \mathbb{X}^{\infty}$ satisfies the Rabier condition (respectively the Kuo-KOS condition, or the Gaffney condition, or the Jelonek condition) when one uses the Rabier function $\nu$ (respectively the Kuo function $\kappa$, or the Gaffney function $\gamma$, or the Jelonek function $\zeta$ ) to generate $\mathcal{N}_{\infty}(f)$ and one has that $z_{0} \notin\left(\tau^{-1}\left(\mathcal{N}_{\infty}(f)\right) \cap \mathbb{X} \mathbb{X}^{\infty}\right)$, where $\mathcal{N}_{\infty}(f)$ is as in Definition 2.3.1.

From Lemma 1.3.11, Definition 2.3.1 and Definition 2.3.2, the above conditions do not depend on the chosen function. In other words, we have that $z_{0} \in \mathbb{X}^{\infty}$ satisfies the Rabier condition $\Leftrightarrow z_{0} \in \mathbb{X}^{\infty}$ satisfies the Kuo-KOS condition $\Leftrightarrow z_{0} \in \mathbb{X}^{\infty}$ satifies the Gaffney condition $\Leftrightarrow z_{0} \in \mathbb{X}^{\infty}$ satisfies the Jelonek condition.

From the definitions of $\mathbb{X}^{\infty}$ and $\tau$ (subsection Notation, page 27), we may reformulate the Definition 2.4.1 as follows: we say that $z_{0} \in \mathbb{X}^{\infty}$ does not satisfy the Rabier condition ${ }^{6}$ if there exist $\left\{\left(x_{j}, f\left(x_{j}\right)\right)\right\}_{j \in \mathbb{N}} \subset \operatorname{graph} f \subset\left(\mathbb{P}^{n} \times \mathbb{K}^{p}\right)$ such that $\lim _{j \rightarrow \infty}\left(x_{j}, f\left(x_{j}\right)\right)=z_{0}$ and $\tau\left(z_{0}\right) \in \mathcal{N}_{\infty}(f)$, where $\mathcal{N}_{\infty}(f)$ is generated by the Rabier function $\nu^{7}$.

\subsection{2 t-regularity}

We start with the definitions of conormal space and relative conormal space. Then, we define the space of the characteristic covectors at infinity and $t$-regularity (Definition 2.4.6).

Let $X \subset \mathbb{K}^{m}$ be a real semi-algebraic subset for $\mathbb{K}=\mathbb{R}$ or a complex algebraic set for $\mathbb{K}=\mathbb{C}$. We denote by $X_{\text {reg }}$ the set of regular points of $X$ and by $X_{\text {sing }}$ the set of singular points of $X$. We assume that $X$ contains at least a regular point.

Definition 2.4.2 (Conormal space). Let

$$
C(X):=\operatorname{closure}\left\{(x, H) \in X_{\text {reg }} \times \check{\mathbb{P}}^{m-1} \mid T_{x} X_{\text {reg }} \subset H\right\} \subset \bar{X} \times \check{\mathbb{P}}^{m-1}
$$

be the conormal modification of $X$, where $\check{\mathbb{P}}^{m-1}$ denotes the set of hyperplanes of dimension $m-1$. Let $\pi: C(X) \rightarrow \bar{X}$ denotes the canonical projection on the first factor.

Definition 2.4.3 (Relative conormal space). Let $g$ be an analytic function defined on a neighbourhood of $X$ in $\mathbb{K}^{m}$. Let $X_{0}$ denote the subset of $X_{\text {reg }}$ where $g$ is a submersion. The relative conormal space of $g$ is defined as follows:

$$
C_{g}(X):=\text { closure }\left\{(x, H) \in X_{0} \times \check{\mathbb{P}}^{m-1} \mid T_{x}\left(g^{-1}(g(x))\right) \subset H\right\} \subset \bar{X} \times \check{\mathbb{P}}^{m-1},
$$

\footnotetext{
${ }^{6}$ respectively Kuo-KOS condition, Gaffney condition, Jelonek condition.

${ }^{7}$ respectively Kuo function $\kappa$, Gaffney function $\gamma$, Jelonek function $\zeta$.
} 
together with the projection $\pi: C_{g}(X) \rightarrow \bar{X}, \pi(x, H)=x$.

For $y \in \bar{X}$ such that $g(y)=0$, we set $C_{g, y}(X):=\pi^{-1}(y)$. The following result shows that $C_{g, y}(X)$ depends on the germ of $g$ at $y$ only up to multiplication by some invertible analytic function germ $\gamma$. It was stated for analytic $X$ but it holds for semi-algebraic or complex algebraic sets.

Lemma 2.4.4 (Tibăr [56, Lemma 1.2.7 and Remark 1.2.8]). Let $\gamma:\left(\mathbb{K}^{m}, y\right) \rightarrow \mathbb{K}$ be an analytic function such that $\gamma(y) \neq 0$. Then $C_{\gamma g, y}(X)=C_{g, y}(X)$.

\section{Characteristic covectors and $t$-regularity}

Let $X \subset \mathbb{K}^{n}$ be a semi-algebraic set for $\mathbb{K}=\mathbb{R}$, or a complex algebraic set for $\mathbb{K}=\mathbb{C}$. Let $f: X \rightarrow \mathbb{K}^{p}$ be a $C^{1}$ semi-algebraic mapping for $\mathbb{K}=\mathbb{R}$ or a restriction of a complex polynomial mapping for $\mathbb{K}=\mathbb{C}$, where $\operatorname{dim} X \geq p$.

As before (page 27), we set $\mathbb{X}:=\overline{\operatorname{graph} f}$ as the closure of the graph of $f$ in $\mathbb{P}^{n} \times \mathbb{K}^{p}$ (via the canonical embedding of $\mathbb{K}^{n} \times \mathbb{K}^{p}$ in $\left.\mathbb{P}^{n} \times \mathbb{K}^{p}\right)$ and set $\mathbb{X}^{\infty}:=\mathbb{X} \cap\left(\mathbb{H}^{\infty} \times \mathbb{K}^{p}\right)$. Note that the points of $\mathbb{X}^{\infty}$ are just the points of the boundary of the graph $f$ in $\mathbb{P}^{n} \times \mathbb{K}^{p}$.

Let $U_{j} \times \mathbb{K}^{p}$ be the affine charts of $\mathbb{P}^{n} \times \mathbb{K}^{p}$, where $U_{j}=\left\{x_{j} \neq 0\right\}, j=0,1, \ldots, n$. Identifying the chart $U_{0}$ with the affine space $\mathbb{K}^{n}$, we have the identification $\left(\mathbb{X} \cap\left(U_{0} \times \mathbb{K}^{p}\right)\right)=$ $\mathbb{X} \backslash \mathbb{X}^{\infty}=\operatorname{graph} f$ and $\mathbb{X}^{\infty}$ is covered by the charts $\left\{\left(U_{1} \times \mathbb{K}^{p}\right), \ldots,\left(U_{n} \times \mathbb{K}^{p}\right)\right\}$.

If $g$ denotes the projection to the variable $x_{0}$ in some affine chart $U_{j} \times \mathbb{K}^{p}$, then the relative conormal $C_{g}\left(\mathbb{X} \backslash \mathbb{X}^{\infty} \cap U_{j} \times \mathbb{K}^{p}\right) \subset \mathbb{X} \times \check{\mathbb{P}}^{n+p-1}$ is well defined, with the projection $\pi(y, H)=y$.

Let us then consider the set $\pi^{-1}\left(\mathbb{X}^{\infty}\right)$ which is well defined for every chart $U_{j} \times \mathbb{K}^{p}$ as a subset of $C_{g}\left(\mathbb{X} \backslash \mathbb{X}^{\infty} \cap U_{j} \times \mathbb{K}^{p}\right)$. The elements of $\mathbb{X}^{\infty}$ differ from one chart to the other by multiplication with a rational function of type $x_{i} / x_{j}$. Since these functions are non zero on $\mathbb{X}^{\infty}$, one has by Lemma 2.4.4 that the definitions coincide at the intersections of the charts. We therefore have:

Definition 2.4.5. The space of characteristic covectors at infinity is the well-defined set $\mathcal{C}^{\infty}:=\pi^{-1}\left(\mathbb{X}^{\infty}\right)$. For some $z_{0} \in \mathbb{X}^{\infty}$, let $\mathcal{C}_{z_{0}}^{\infty}:=\pi^{-1}\left(z_{0}\right)$.

Let $\tau: \mathbb{P}^{n} \times \mathbb{K}^{p} \rightarrow \mathbb{K}^{p}$ denote the second projection. We define the relative conormal space $C_{\tau}\left(\mathbb{P}^{n} \times \mathbb{K}^{p}\right)$ like in Definition 2.4.3 where the function $g$ is replaced by the mapping $\tau$.

Definition 2.4.6 (t-regularity). We say that $f$ is $t$-regular at $z_{0} \in \mathbb{X}^{\infty}$ if $C_{\tau}\left(\mathbb{P}^{n} \times \mathbb{K}^{p}\right) \cap \mathcal{C}_{z_{0}}^{\infty}=\emptyset$.

Remark 2.4.7. The $t$-regularity was defined by Siersma and Tibăr [49] for polynomial functions $f: \mathbb{K}^{n} \rightarrow \mathbb{K}$ (see also Parusiński [42]) and later used for instance in Tibăr [53, 54, 55, 56]. 


\section{$t$-regularity interpretation}

Let $f=\left(f_{1}, \ldots, f_{p}\right): \mathbb{K}^{n} \rightarrow \mathbb{K}^{p}$ be a $C^{1}$ semi-algebraic mapping for $\mathbb{K}=\mathbb{R}$ or a complex polynomial mapping for $\mathbb{K}=\mathbb{C}$. Consider $z_{0} \in \mathbb{X}^{\infty}$. Up to some linear change of coordinate we may assume that $z_{0} \in \mathbb{X}^{\infty} \cap\left(U_{n} \times \mathbb{K}^{p}\right)$.

In the intersection of charts $\left(U_{0} \cap U_{n}\right) \times \mathbb{K}^{p}$, let $x_{1}=y_{1} / y_{0}, \ldots, x_{n-1}=y_{n-1} / y_{0}, x_{n}=1 / y_{0}$, where $\left(x_{1}, \ldots, x_{n}\right)$ are the coordinates in $U_{0}$ and $\left(y_{0}, \ldots, y_{n-1}\right)$ are those in $U_{n}$. We use coordinates $\left(t_{1}, \ldots, t_{p}\right)$ for $\mathbb{K}^{p}$. With these notations, for each $i \in\{1, \ldots, p\}$ the following equations hold:

$$
F_{i}(y, t)=F_{i}\left(y_{0}, y_{1}, \ldots, y_{n-1}, t_{1}, \ldots, t_{p}\right):=f_{i}\left(\frac{y_{1}}{y_{0}}, \ldots, \frac{y_{n-1}}{y_{0}}, \frac{1}{y_{0}}\right)-t_{i},
$$

and we set $F(y, t):=\left(F_{1}(y, t), \ldots, F_{p}(y, t)\right)$.

From the above equations, one obtains the equality $\mathbb{X} \cap\left(\left(U_{0} \cap U_{n}\right) \times \mathbb{K}^{p}\right)=\bigcap_{i=1}^{p}\left\{F_{i}(y, t)=0\right\}$.

Denote by $\overrightarrow{n_{0}}=(1,0, \ldots, 0) \in \mathbb{K}^{n} \times \mathbb{K}^{p}$ the normal vector to the hypersurface $\left\{y_{0}=\right.$ constant $\}$ and for each $i=1, \ldots, p$, let us consider a normal vector to $\left\{F_{i}=0\right\}$ at $(y, t) \in$ $\mathbb{X} \cap\left(\left(U_{0} \cap U_{n}\right) \times \mathbb{K}^{p}\right)$, as follows:

$$
\overrightarrow{n_{i}}(y, t)=\nabla F_{i}(y, t)=\left(\nabla_{n} F_{i}(y, t), \nabla_{p} F_{i}(y, t)\right)
$$

where

$$
\begin{aligned}
\nabla_{n} F_{i}(y, t):=\left(\frac{\partial F_{i}}{\partial y_{0}}(y, t), \ldots, \frac{\partial F_{i}}{\partial y_{n-1}}(y, t)\right) \text { and } & \\
\qquad \nabla_{p} F_{i}(y, t) & :=\left(\frac{\partial F_{i}}{\partial t_{1}}(y, t), \ldots, \frac{\partial F_{i}}{\partial t_{p}}(y, t)\right) .
\end{aligned}
$$

By Definition 2.4.6, $f$ is not $t$-regular at $z_{0} \in \mathbb{X}^{\infty}$ if and only if there exists a sequence $\left\{\left(y_{k}, t_{k}\right)\right\}_{k \in \mathbb{N}} \subset \mathbb{X} \cap\left(\left(U_{0} \cap U_{n}\right) \times \mathbb{K}^{p}\right)$ such that $\left(y_{k}, t_{k}\right) \rightarrow z_{0}$ and the tangent hyperplanes to the fibres of $g_{\mid \mathbb{X}}$ at $\left(y_{k}, t_{k}\right)$ tend to a hyperplane $H$ such that its normal line has a direction of the form $\left[0: \cdots: 0: b_{1}: \cdots: b_{p}\right]$ in $\mathbb{P}^{n+p-1}$. More explicitly, there exists a sequence

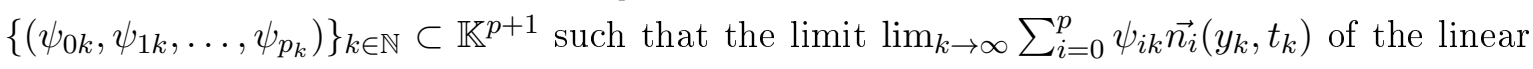
combination of normal vectors $\overrightarrow{n_{i}}$ has the direction $\vec{n}_{H}=\left[0: 0: \ldots: 0: b_{1}: \ldots: b_{p}\right] \in \mathbb{P}^{n+p-1}$.

\subsubsection{Regularity conditions and the $t$-regularity}

Let $f: \mathbb{K}^{n} \rightarrow \mathbb{K}^{p}$ be a $C^{1}$ semi-algebraic mapping for $\mathbb{K}=\mathbb{R}$, or a complex polynomial mapping for $\mathbb{K}=\mathbb{C}$, where $n \geq p$. We have defined four regularity conditions for $f$ : Rabier condition, Kuo-KOS condition, Gaffney condition and Jelonek condition. Due to results of Jelonek [24] and Kurdyka, Orro and Simon [29], we know that these regularity conditions are equivalent, see Definition 2.3.1 and Definition 2.3.2.

These regularity conditions are asymptotic conditions that depend on the behaviour of 
the fibres of $f$ and on the Jacobian of $f$. Originally, they have been defined on the affine space $\mathbb{K}^{n}$. Then, in $\S 2.4 .1$, we reformulate these conditions in a localized version on a point of the boundary of the graph of $f$ in $\mathbb{P}^{n} \times \mathbb{K}^{p}$. On the other hand, we have defined in $\S 2.4 .2$ the $t$-regularity (Definition 2.4.6), a geometric condition that depends on the limits of hyperplanes and that has been defined on a local context, locally in a point of the boundary of the graph of $f$. Under this reformulation of $\S 2.4 .1$ and definitions of $\S 2.4 .2$, we have:

Theorem 2.4.8. Let $f: \mathbb{K}^{n} \rightarrow \mathbb{K}^{p}$ be a $C^{1}$ semi-algebraic mapping for $\mathbb{K}=\mathbb{R}$, or a complex polynomial mapping for $\mathbb{K}=\mathbb{C}$, where $n \geq p$. Consider $z_{0} \in \mathbb{X}^{\infty}$. Then $f$ is t-regular at $z_{0}$ if and only if $z_{0}$ satisfies the Rabier condition (or equivalently the Kuo-KOS condition, or the Gaffney condition, or the Jelonek condition).

Proof. One may assume (eventually after some linear change of coordinates) that $z_{0} \in \mathbb{X}^{\infty} \cap$ $\left(U_{n} \times \mathbb{K}^{p}\right)$ and that $\left|x_{n}\right| \geq\left|x_{i}\right|$, for $x=\left(x_{1}, \ldots, x_{n}\right) \in \mathbb{K}^{n}$ in some neighbourhood of $z_{0}$ and $i=1, \ldots, n-1$.

“ $\Rightarrow$ ". From Definition 2.4.1, if $z_{0}$ does not satisfy the Rabier condition and therefore $t_{0}:=$ $\tau\left(z_{0}\right) \in \mathcal{N}_{\infty}(f)^{8}$, then there exist sequences $\left\{\mathrm{x}_{k}:=\left(x_{1 k}, \ldots, x_{n k}\right)\right\}_{k \in \mathbb{N}} \subset \mathbb{K}^{n}$ and $\left\{\psi_{k}=\right.$ $\left.\left(\psi_{1 k}, \ldots, \psi_{p k}\right)\right\}_{k \in \mathbb{N}} \subset \mathbb{K}^{p}$ with $\left\|\psi_{k}\right\|=1$ and $\psi_{k} \rightarrow \psi$, such that $\left(\mathrm{x}_{k}, f\left(\mathrm{x}_{k}\right)\right) \rightarrow z_{0}$ and

$$
\left\|\mathrm{x}_{k}\right\|\left\|\left(\sum_{i=1}^{p} \psi_{i k} \frac{\partial f_{i}}{\partial x_{1}}\left(\mathrm{x}_{k}\right), \ldots, \sum_{i=1}^{p} \psi_{i k} \frac{\partial f_{i}}{\partial x_{n}}\left(\mathrm{x}_{k}\right)\right)\right\| \rightarrow 0
$$

Since for large enough $k$ we have $\left|x_{n k}\right| \geq\left|x_{i k}\right|, i=1, \ldots, n-1$, we can replace in equation (2.9) the number $\left\|\mathrm{x}_{k}\right\|$ by the number $\left|x_{n k}\right|$, then multiply each coordinate of the vector by $x_{n k}$

Under the notations of the subsection " $t$-regularity interpretation (page 30)" and by changing coordinates within $U_{0} \cap U_{n}$, one has $y_{0}=1 / x_{n}, y_{1}=x_{1} / x_{n}, \ldots, y_{n-1}=x_{n-1} / x_{n}$, and the relations:

$$
\left\{\begin{array}{lll}
\frac{\partial F_{j}}{\partial y_{i}}(y, t)=x_{n} \frac{\partial f_{j}}{\partial x_{i}}(x), & & 1 \leq i \leq n-1,1 \leq j \leq p, \\
\frac{\partial F_{j}}{\partial t_{l}}(y, t)=-\delta_{l, j}, & & 1 \leq j, l \leq p, \\
\frac{\partial F_{j}}{\partial y_{0}}(y, t)=-x_{n}\left(x_{1} \frac{\partial f_{j}}{\partial x_{1}}(x)+\ldots+x_{n} \frac{\partial f_{j}}{\partial x_{n}}(x)\right), & & 1 \leq j \leq p .
\end{array}\right.
$$

From the above interpretations, the condition (2.9) implies:

$$
\left\|\left(\sum_{i=1}^{p} \psi_{i k} \frac{\partial F_{i}}{\partial y_{1}}\left(\mathrm{y}_{k}, \mathrm{t}_{k}\right), \ldots, \sum_{i=1}^{p} \psi_{i k} \frac{\partial F_{i}}{\partial y_{n-1}}\left(\mathrm{y}_{k}, \mathrm{t}_{k}\right)\right)\right\| \rightarrow 0
$$

where $\mathrm{y}_{k}=\left(y_{0 k}, y_{1 k}, \ldots, y_{n-1 k}\right)=\left(1 / x_{n k}, x_{1 k} / x_{n k}, \ldots, x_{n-1 k} / x_{n k}\right)$ and $\mathrm{t}_{k}:=f\left(\mathrm{x}_{k}\right)$.

\footnotetext{
${ }^{8} \tau$ is as before, i.e., $\tau: \mathbb{P}^{n} \times \mathbb{K}^{p}$ is just the second projection and $\mathcal{N}_{\infty}(f)$ is as in Definition 2.4.1.
} 
The following vector

$$
\vec{n}_{H_{k}}:=\left(0, \sum_{i=1}^{p} \psi_{i k} \frac{\partial F_{i}}{\partial y_{1}}\left(\mathrm{y}_{k}, \mathrm{t}_{k}\right), \ldots, \sum_{i=1}^{p} \psi_{i k} \frac{\partial F_{i}}{\partial y_{n-1}}\left(\mathrm{y}_{k}, \mathrm{t}_{k}\right),-\psi_{1 k}, \ldots,-\psi_{p k}\right)
$$

is a linear combination of the normal vectors $\vec{n}_{i}$ defined in the subsection " $t$-regularity interpretation (page 30)" with coefficients $\psi_{i k}$. Consequently, the hyperplane $H_{k}$ normal to $\vec{n}_{H_{k}}$ is tangent to the levels of the function $g_{\mid \mathbb{X}}$.

Therefore, from equation (2.11) and the condition on $\left\{\psi_{k}\right\}$ (beginning of the proof), we obtain the following limit

$$
\vec{n}_{H_{k}} \rightarrow \vec{n}=\left[0: 0: \ldots: 0: \psi_{1}: \ldots: \psi_{p}\right]
$$

which shows that the limit tangent hyperplane $H=\lim _{k \rightarrow \infty} H_{k}$, to which $\vec{n}$ is normal, belongs to $\mathcal{C}_{z_{0}}^{\infty}$. This implies that $f$ is not $t$-regular at $z_{0}$.

" $\Leftarrow$ ". Reciprocally, let $z_{0} \in \mathbb{X}^{\infty}$ be not $t$-regular. Then there exist some sequence of points $\left\{\left(\mathrm{y}_{k}, \mathrm{t}_{k}\right)\right\}_{k \in \mathbb{N}} \subset \mathbb{X} \cap\left(\left(U_{0} \cap U_{n}\right) \times \mathbb{K}^{p}\right)$ tending to $z_{0}$, and a sequence of hyperplanes $H_{k}$ tangent to the levels of $g$ at $\left(\mathrm{y}_{k}, \mathrm{t}_{k}\right)$, such that $H_{k} \rightarrow H \in \mathcal{C}_{z_{0}}^{\infty}$. This means that there exist sequences $\left\{\tilde{\psi}_{k}=\left(\tilde{\psi}_{1 k}, \ldots, \tilde{\psi}_{p k}\right)\right\}_{k \in \mathbb{N}} \subset \mathbb{K}^{p}$ and $\left\{\lambda_{k}\right\}_{k \in \mathbb{N}} \subset \mathbb{K}$ such that $\vec{n}_{H_{k}}=\lambda_{k} \vec{n}_{0}\left(\mathrm{y}_{k}, \mathrm{t}_{k}\right)+$ $\sum_{i} \tilde{\psi}_{i k} \vec{n}_{i}\left(\mathrm{y}_{k}, \mathrm{t}_{k}\right)$ and one has the equality $\lim _{k \rightarrow \infty} \vec{n}_{H_{k}}=\left[0: 0: \ldots: 0: \tilde{\psi}_{1}: \ldots: \tilde{\psi}_{p}\right]$, where $\left(\tilde{\psi}_{1}, \ldots, \tilde{\psi}_{p}\right) \neq(0, \ldots, 0)$. By assumption, the $\vec{n}_{H_{k}}$ is the vector:

$$
\left(\lambda_{k}+\sum_{i=1}^{p} \tilde{\psi}_{i k} \frac{\partial F_{i}}{\partial y_{0}}\left(\mathrm{y}_{k}, \mathrm{t}_{k}\right), \sum_{i=1}^{p} \tilde{\psi}_{i k} \frac{\partial F_{i}}{\partial y_{1}}\left(\mathrm{y}_{k}, \mathrm{t}_{k}\right), \ldots, \sum_{i=1}^{p} \tilde{\psi}_{i k} \frac{\partial F_{i}}{\partial y_{n-1}}\left(\mathrm{y}_{k}, \mathrm{t}_{k}\right),-\tilde{\psi}_{1 k}, \ldots,-\tilde{\psi}_{p k}\right)
$$

We may actually take $\lambda_{k}:=-\sum_{i=1}^{p} \tilde{\psi}_{i k} \frac{\partial F_{i}}{\partial y_{0}}\left(\mathrm{y}_{k}, \mathrm{t}_{k}\right)$ and after dividing out by $\mu_{k}:=$ $\left\|\left(\tilde{\psi}_{1 k}, \ldots, \tilde{\psi}_{p k}\right)\right\|$, we get that $\lim _{k \rightarrow \infty} \vec{n}_{H_{k}}=\left(0,0, \ldots, 0, \psi_{1}, \ldots, \psi_{p}\right)$ where $\psi_{i k}:=\frac{\tilde{\psi}_{i k}}{\mu_{k}}$ and $\left\|\left(\psi_{1 k}, \ldots, \psi_{p k}\right)\right\|=1$. This implies that:

$$
\lim _{k \rightarrow \infty} \sum_{i=1}^{p} \psi_{i k} \frac{\partial F_{i}}{\partial y_{j}}\left(\mathrm{y}_{k}, \mathrm{t}_{k}\right)=0
$$

for any $1 \leq j \leq n-1$.

From (2.10), this is equivalent to:

$$
\lim _{k \rightarrow \infty} x_{n k} \sum_{i=1}^{p} \psi_{i k} \frac{\partial f_{i}}{\partial x_{j}}\left(\mathrm{x}_{k}\right)=0
$$

and one has $\left|x_{n k}\right| \geq \frac{1}{\sqrt{n}}\left\|\mathrm{x}_{k}\right\|$ for large enough $k$. Therefore, in order to get the limit (2.9) it remains to prove that (2.13) is true for $j=n$. The rest of our argument is devoted to this proof. 
It follows from relations $(2.10)$ that $x_{n} \frac{\partial f_{i}}{\partial x_{n}}(x)=-\sum_{j=0}^{n-1} y_{j} \frac{\partial F_{i}}{\partial y_{j}}(y, t)$ and therefore:

$$
\sum_{i=1}^{p} \psi_{i k} x_{n k} \frac{\partial f_{i}}{\partial x_{n}}\left(\mathrm{x}_{k}\right)=-\sum_{j=1}^{n-1} \sum_{i=1}^{p} y_{j k} \psi_{i k} \frac{\partial F_{i}}{\partial y_{j}}\left(\mathrm{y}_{k}, \mathrm{t}_{k}\right)-\sum_{i=1}^{p} \psi_{i k} y_{0 k} \frac{\partial F_{i}}{\partial y_{0}}\left(\mathrm{y}_{k}, \mathrm{t}_{k}\right) .
$$

We will show that both terms of the right hand side tend to zero, which implies that (2.13) is true for $j=n$. First we have:

$$
\left|\sum_{j=1}^{n-1} \sum_{i=1}^{p} y_{j k} \psi_{i k} \frac{\partial F_{i}}{\partial y_{j}}\left(\mathrm{y}_{k}, \mathrm{t}_{k}\right)\right| \leq\left\|\frac{\mathrm{x}_{k}}{x_{n k}}\right\|\left\|\left(\sum_{i=1}^{p} \psi_{i k} \frac{\partial F_{i}}{\partial y_{1}}\left(\mathrm{y}_{k}, \mathrm{t}_{k}\right), \ldots, \sum_{i=1}^{p} \psi_{i k} \frac{\partial F_{i}}{\partial y_{n-1}}\left(\mathrm{y}_{k}, \mathrm{t}_{k}\right)\right)\right\| .
$$

Since by hypothesis one has $\left|y_{j k}\right|=\left|\frac{x_{j k}}{x_{n k}}\right| \leq 1$ for large enough $k$, we get from (2.13) that the right hand side tends to 0 as $k \rightarrow \infty$.

Let us assume that the following inequality holds for large enough $k \gg 1$, the proof of which will be given below:

$$
\left\|\sum_{i=1}^{p} \psi_{i k} y_{0 k} \frac{\partial F_{i}}{\partial y_{0}}\right\| \ll\left\|\left(\sum_{i=1}^{p} \psi_{i k} \frac{\partial F_{i}}{\partial y_{1}}, \ldots, \sum_{i=1}^{p} \psi_{i k} \frac{\partial F_{i}}{\partial y_{n-1}}, \sum_{i=1}^{p} \psi_{i k} \frac{\partial F_{i}}{\partial t_{1}}, \ldots, \sum_{i=1}^{p} \psi_{i k} \frac{\partial F_{i}}{\partial t_{p}}\right)\right\| .
$$

Then, by using (2.12), (2.14) and the equality $\sum_{i=1}^{p} \psi_{i k} \frac{\partial F_{i}}{\partial t_{j}}=-\psi_{j k}$ for any $1 \leq j \leq p$ (implied by (2.10)), we get:

$$
\left\|\sum_{i=1}^{p} \psi_{i k} y_{0 k} \frac{\partial F_{i}}{\partial y_{0}}\right\| \ll\left\|\psi_{k}\right\|=1
$$

which shows that $\lim _{k \rightarrow \infty} \sum_{i=1}^{p}\left\|\psi_{i k} y_{0 k} \frac{\partial F_{i}}{\partial y_{0}}\left(\mathrm{y}_{k}, \mathrm{t}_{k}\right)\right\|=0$. This completes our proof of the relation (2.9) showing that $z_{0}$ does not satisfy the Rabier condition.

Let us now give the proof of (2.14). If this were not true, there exists $\delta>0$ such that for $k \gg 1$ one has:

$$
\frac{\left\|\sum_{i=1}^{p} \psi_{i k} y_{0 k} \frac{\partial F_{i}}{\partial y_{0}}\left(\mathrm{y}_{k}, \mathrm{t}_{k}\right)\right\|}{\left\|\left(\sum_{i=1}^{p} \psi_{i k} \frac{\partial F_{i}}{\partial y_{1}}, \ldots, \sum_{i=1}^{p} \psi_{i k} \frac{\partial F_{i}}{\partial y_{n-1}}, \sum_{i=1}^{p} \psi_{i k} \frac{\partial F_{i}}{\partial t_{1}}, \ldots, \sum_{i=1}^{p} \psi_{i k} \frac{\partial F_{i}}{\partial t_{p}}\right)\left(\mathrm{y}_{k}, \mathrm{t}_{k}\right)\right\|}>\delta .
$$

Then the set $\mathcal{W}=\left\{((y, t), \psi) \in\left(\left(U_{n} \cap U_{0}\right) \times \mathbb{K}^{p} \times \mathbb{K}^{p}\right) \cap\left(\mathbb{X} \times S_{1}^{p-1}\right) \mid(2.15)\right.$ holds for $\left.((y, t), \psi)\right\}$ is a semi-algebraic set. We have $\left(\left(\mathrm{y}_{k}, \mathrm{t}_{k}\right), \psi_{k}\right) \in \mathcal{W}$ for $k \gg 1$, thus $\left(z_{0}, \psi\right) \in \overline{\mathcal{W}}$. Then, by Curve Selection Lemma (Milnor $[34, \S 3]$ ) there exists an analytic curve $\lambda=$ $(\phi, \psi):\left[0, \varepsilon\left[\rightarrow \overline{\mathcal{W}}\right.\right.$ such that $\lambda(] 0, \varepsilon[) \subset \mathcal{W}$ and $\lambda(0)=\left(z_{0}, \psi\right)$. We denote $\phi(s)=$ $\left(y_{0}(s), y_{1}(s), \ldots, y_{n-1}(s), t_{1}(s), \ldots, t_{p}(s)\right)$ and $\psi(s)=\left(\psi_{1}(s), \ldots, \psi_{p}(s)\right)$. Since $F(\phi(s)) \equiv 0$, 
we have:

$$
0=\frac{d}{d s} F(\phi(s))=y_{0}^{\prime}(s) \frac{\partial F}{\partial y_{0}}(\phi(s))+\sum_{i=1}^{n-1} y_{i}^{\prime}(s) \frac{\partial F}{\partial y_{i}}(\phi(s))+\sum_{i=1}^{p} t_{i}^{\prime}(s) \frac{\partial F}{\partial t_{i}}(\phi(s)) .
$$

Multiplying by $\psi(s)$ we obtain:

$$
-y_{0}^{\prime}(s) \sum_{i=1}^{p} \psi_{i}(s) \frac{\partial F_{i}}{\partial y_{0}}(\phi(s))=\sum_{j=1}^{n-1} y_{j}^{\prime}(s) \sum_{i=1}^{p} \psi_{i}(s) \frac{\partial F_{i}}{\partial y_{j}}(\phi(s))+\sum_{j=1}^{p} t_{j}^{\prime}(s) \sum_{i=1}^{p} \psi_{i}(s) \frac{\partial F_{i}}{\partial t_{j}}(\phi(s)) .
$$

Since $\phi$ is analytic, thus bounded at $s=0$, by applying the Cauchy-Schwarz inequality one finds a constant $C>0$ such that:

$$
\begin{aligned}
& \mid\left(y_{0}^{\prime}(s) \sum_{i=1}^{p} \psi_{i}(s) \frac{\partial F_{i}}{\partial y_{0}}(\phi(s)) \mid \leq\right. \\
& C\left\|\left(\sum_{i=1}^{p} \psi_{i} \frac{\partial F_{i}}{\partial y_{1}}(\phi), \ldots, \sum_{i=1}^{p} \psi_{i} \frac{\partial F_{i}}{\partial y_{n-1}}(\phi), \sum_{i=1}^{p} \psi_{i} \frac{\partial F_{i}}{\partial t_{1}}(\phi), \ldots, \sum_{i=1}^{p} \psi_{i} \frac{\partial F_{i}}{\partial t_{p}}(\phi)\right)(s)\right\| .
\end{aligned}
$$

We have $l:=\operatorname{ord}_{s} y_{0}^{\prime}(s) \geq 0$ and $\operatorname{ord}_{s} y_{0}(s)=l+1 \geq 1$ since $y_{0}(0)=0$, thus $\left|y_{0}(s) \sum_{i=1}^{p} \psi_{i}(s) \frac{\partial F_{i}}{\partial y_{0}}(\phi(s))\right| \ll\left|y_{0}^{\prime}(s) \sum_{i=1}^{p} \psi_{i}(s) \frac{\partial F_{i}}{\partial y_{0}}(\phi(s))\right|$, which, together with (2.16), gives:

$$
\begin{aligned}
&\left\|\sum_{i=1}^{p} \psi_{i}(s) y_{0}(s) \frac{\partial F_{i}}{\partial y_{0}}(\phi(s))\right\| \ll \\
&\left\|\left(\sum_{i=1}^{p} \psi_{i} \frac{\partial F_{i}}{\partial y_{1}}(\phi), \ldots, \sum_{i=1}^{p} \psi_{i} \frac{\partial F_{i}}{\partial y_{n-1}}(\phi), \sum_{i=1}^{p} \psi_{i} \frac{\partial F_{i}}{\partial t_{1}}(\phi), \ldots, \sum_{i=1}^{p} \psi_{i} \frac{\partial F_{i}}{\partial t_{p}}(\phi)\right)(s)\right\| .
\end{aligned}
$$

This contradicts our assumption that $(\phi(s), \psi(s)) \in \mathcal{W}$, for $s \in] 0, \varepsilon[$.

As a direct consequence of the Theorem 2.4.8, one has:

Corollary 2.4.9. Let $f: \mathbb{K}^{n} \rightarrow \mathbb{K}^{p}$ as in Theorem 2.4.8. If we set $\mathcal{N} \mathcal{T}_{\infty}(f):=\left\{t_{0}=\right.$ $\tau\left(z_{0}\right) \in \mathbb{K}^{p} \mid z_{0}$ is not t-regular and $\left.z_{0} \in \mathbb{X}^{\infty}\right\}$, then $\mathcal{N}_{\infty}(f)=\mathcal{N} \mathcal{T}_{\infty}(f)$, where $\mathcal{N}_{\infty}(f)$ is as in Definition 2.3.1 and Definition 2.3.2.

REMARK 2.4.10. In 2.3.4, has been observed that for functions $f: \mathbb{K}^{n} \rightarrow \mathbb{K}$, Rabier, Kuo-KOS, Gaffney and Jelonek conditions coincide with Malgrange condition (Definition 2.2.10). On the other hand, we have seen in $\S 2.2$ (page 23) that for polynomial functions $f: \mathbb{K}^{n} \rightarrow \mathbb{K}[49$, Siersma and Tibăr] and [43, Parusiński] proved that Malgrange condition is equivalent to $t$ regularity. Therefore, Theorem 2.4.8 is a far-reaching extension of the equivalence "Malgrange condition $\Leftrightarrow t$-regularity" established for polynomial functions. 


\section{$2.5 \rho$-regularity}

We introduce $\rho$-regularity, which is a regularity condition at infinity based on a control function $\rho$. The $\rho$-regularity enables to define the set of non $\rho$-regular values, denoted here by $S(f)$, and the set $A_{\rho}(f)=f(\operatorname{Sing} f) \cup S(f)$.

The interest in this regularity condition is that, as well as for the regularity conditions considered in 2.3, we can obtain an asymptotic Morse-Sard type theorem for the $\rho$-regularity. This Morse-Sard type theorem and other asymptotic results for the $\rho$-regularity will be presented in Chapter 3.

In this section, we define $\rho$-regularity $(\$ 2.5 .1)$ and show that the equivalent conditions considered in sections 2.3 and 2.4 imply $\rho_{E}$-regularity (§2.5.2).

\subsection{1 $\rho$-regularity at infinity}

Let $K \subset \mathbb{K}^{n}$ be some compact (eventually empty) set and let $\rho: \mathbb{K}^{n} \backslash K \rightarrow \mathbb{R}_{\geq 0}$ be a proper submersion.

Let $f: X \rightarrow \mathbb{K}^{p}$ be a $C^{1}$-mapping, where $X \subset \mathbb{K}^{n}$ is a submanifold. As before, we use the following definitions and notations: we set $\mathbb{X}:=\overline{\operatorname{graph} f}$ the closure of the graph of $f$ in $\mathbb{P}^{n} \times \mathbb{K}^{p}$ (via the embedding of $\mathbb{K}^{n}$ in $\mathbb{P}^{n}$ ) and we set $\mathbb{X}^{\infty}:=\mathbb{X} \cap\left(\mathbb{H}^{\infty} \times \mathbb{K}^{p}\right.$ ), where $\mathbb{H}^{\infty}=\left\{\left[x_{0}: \ldots: x_{n}\right] \in \mathbb{P}^{n} \mid x_{0}=0\right\}$ (see the subsection Notation (page 27)). We denote by $\tau: \mathbb{P}^{n} \times \mathbb{K}^{p} \rightarrow \mathbb{K}^{p}$ the second projection, that is $\tau(\mathrm{x}, \mathrm{t})=\mathrm{t}$, for any $(\mathrm{x}, \mathrm{t}) \in \mathbb{P}^{n} \times \mathbb{K}^{p}$.

Under these notations, we have:

Definition 2.5.1 ( $\rho$-regularity at infinity). We say that $f$ is $\rho$-regular at $z_{0} \in \mathbb{X}^{\infty}$ if there is an open neighbourhood $U \subset \mathbb{P}^{n} \times \mathbb{K}^{p}$ of $z_{0}$ and an open neighbourhood $D \subset \mathbb{K}^{p}$ of $\tau\left(z_{0}\right)$ such that, for all $t \in D$, the fibre $f^{-1}(t) \cap U$ intersects all the levels of the restriction $\rho_{\mid U \cap \mathbb{K}^{n}}$ and this intersection is transversal.

We call Milnor set the critical locus of the map $(f, \rho)$ and denote it by $\mathcal{M}(f)$, that is $\mathcal{M}(f)=\{x \in X \mid x \in \operatorname{Sing}(f, \rho)\}$.

We say that the fibre $f^{-1}\left(t_{0}\right)$ is $\rho$-regular at infinity if $f$ is $\rho$-regular at all points $z_{0} \in$ $\mathbb{X}^{\infty} \cap \tau^{-1}\left(t_{0}\right)$. We call:

$$
S(f):=\left\{t_{0} \in \mathbb{K}^{p} \mid \exists\left\{x_{j}\right\}_{j \in \mathbb{N}} \subset \mathcal{M}(f), \lim _{j \rightarrow \infty}\left\|x_{j}\right\|=\infty \text { and } \lim _{j \rightarrow \infty} f\left(x_{j}\right)=t_{0}\right\},
$$

the set of asymptotic $\rho$-nonregular values. We denote $A_{\rho}:=f(\operatorname{Sing} f) \cup S(f)$ and call it the $\rho$-bifurcation set.

REMARK 2.5.2. The definition of $\rho$-regularity at infinity of a fibre $f^{-1}\left(t_{0}\right)$ does not depend on any proper extension of $f$, since it is equivalent to the following: for any sequence $\left\{x_{k}\right\}_{k \in \mathbb{N}} \subset$ $\mathbb{K}^{n},\left\|x_{k}\right\| \rightarrow \infty, f\left(x_{k}\right) \rightarrow t_{0}$, there exists some $k_{0}$ such that, if $k \geq k_{0}$ then $f$ is transversal to $\rho$ at $x_{k}$. 
The transversality of the fibres of $f$ to the levels of $\rho$ is a "Milnor type" condition. In the case that $\rho$ is the Euclidean norm, denoted in this thesis by $\rho_{E}$, the condition of transversality of the fibres of $f$ to the levels of $\rho_{E}$ (in this case spheres) has been used by Milnor in the local study of singular functions $[34, \S 2, \S 4, \S 5]$.

In the global context of complex polynomial functions, transversality to big spheres (i.e. $\rho_{E}$-regularity, in our definition) was used in Broughton [5, page 229] and later in Némethi and Zaharia [40], where it is called $M$-tame condition, (see Definition 2.2.11). The name "Milnor set" occurs in [40] too.

Distance functions like $\rho$ are also central ingredients in defining regular stratifications, e.g. Bekka [1], Kuo [27], Mather [32].

EXAMPLE 2.5.3. Let $\rho: \mathbb{K}^{n} \rightarrow \mathbb{R}_{\geq 0}, \rho(x)=\left(\sum_{i=1}^{n}\left|x_{i}\right|^{2 p_{i}}\right)^{1 / 2 p}$, where $\left(w_{1}, \ldots, w_{n}\right) \in \mathbb{N}^{n}$, $p=\operatorname{lcm}\left\{w_{1}, \ldots, w_{n}\right\}$ and $w_{i} p_{i}=p, \forall i$. Here $\operatorname{lcm}\left\{w_{1}, \ldots, w_{n}\right\}$ means the least common multiple $\left\{w_{1}, \ldots, w_{n}\right\}$. This function is "adapted" to polynomials which are quasihomogeneous of type $\left(w_{1}, \ldots, w_{n}\right)$. By using it, one can show that a value $c \in \mathbb{K}$ is bifurcation value for such a polynomial if and only if $c$ is a critical value of $f$ (hence only the value 0 can be a bifurcation). Namely, let $E_{r}:=\left\{x \in \mathbb{K}^{n} \mid \rho(x)<r\right\}$ for some $r>0$. Then the local Milnor fibre of $f$ at $0 \in \mathbb{K}^{n}$ (i.e. $f^{-1}(c) \cap E_{\varepsilon}$, for some small enough $\varepsilon$ and $0<|c| \ll \varepsilon$ ) is diffeomorphic to the global fibre $f^{-1}(c)$, since $f^{-1}(c)$ is transversal to $\partial \overline{E_{r}}, \forall r \geq \varepsilon$.

\subsection{2 $t$-regularity and $\rho_{E}$-regularity}

We shall show that $t$-regularity implies $\rho_{E^{-}}$-regularity in two contexts. Firstly, for $C^{1}$ semialgebraic mappings $f: X \rightarrow \mathbb{R}^{p}$, where $X$ is a smooth semi-algebraic set (Proposition 2.5.4). Next, for $C^{1}$ semi-algebraic mappings $f: \mathbb{R}^{n} \rightarrow \mathbb{R}^{p}$ (Proposition 2.5.5).

We remark that these results, more precisely $t$-regularity $\Rightarrow \rho_{E}$-regularity, extend a result proved for polynomial functions in Tibăr [55] (see also Păunescu and Zaharia [45] and our $\S 2.2)$.

We begin with:

Proposition 2.5.4. Let $X \subset \mathbb{R}^{n}$ be semi-algebraic and let $f: X \rightarrow \mathbb{R}^{p}$ be a $C^{1}$ semi-algebraic mapping, where $\operatorname{dim} X>p$. If $f$ is t-regular at $z_{0} \in \mathbb{X}^{\infty}$ then $f$ is $\rho_{E}$-regular at $z_{0}$.

Proof. We may assume without loss of generality that $z_{0}=([0: 0: \ldots: 1], 0, \ldots 0)$. Let $d^{\infty}: \mathbb{X} \cap U_{n} \times \mathbb{R}^{p} \rightarrow \mathbb{R}_{\geq 0},(y, t) \mapsto \frac{y_{0}^{2}}{y_{1}^{2}+\ldots+y_{n-1}^{2}+1}$ and note that $d^{\infty}(y, t)=\frac{1}{\rho_{E}^{2}(\mathrm{x})}$. As usual, we denote by $g$ the projection to the variable $y_{0}$. At $z_{0}$, the functions $g^{2}$ and $d^{\infty}$ differ by a unit, they have the same zero locus $\mathbb{X}^{\infty}$ and the same levels. Therefore $\mathcal{C}_{z_{0}}^{\infty}=C_{g, z_{0}}\left(\mathbb{X} \backslash \mathbb{X}^{\infty} \cap\right.$ $\left.\left(U_{n} \times \mathbb{R}^{p}\right)\right)=C_{g^{2}, z_{0}}\left(\mathbb{X} \backslash \mathbb{X}^{\infty} \cap\left(U_{n} \times \mathbb{R}^{p}\right)\right)=C_{d^{\infty}, z_{0}}\left(\mathbb{X} \backslash \mathbb{X}^{\infty} \cap\left(U_{i} \times \mathbb{R}^{p}\right)\right)$, where the last equality follows by Lemma 2.4.4.

The $t$-regularity at $z_{0}$ (Definition 2.4.6) is therefore equivalent to:

$$
C_{\tau}\left(\mathbb{P}^{n} \times \mathbb{R}^{p}\right) \cap C_{d^{\infty}, z_{0}}\left(\mathbb{X} \backslash \mathbb{X}^{\infty} \cap U_{i} \times \mathbb{R}^{p}\right)=\emptyset
$$


which implies that, in some neighbourhood of $z_{0}$ intersected with $\mathbb{R}^{n}$, the fibres $\{\tau=$ const. $\}$ are transverse to the levels of the function $d^{\infty}$, which coincide with the levels of the function $\rho_{E} \cdot$

For maps defined on $\mathbb{R}^{n}$, we have:

Proposition 2.5.5. Let $f=\left(f_{1}, \ldots, f_{p}\right): \mathbb{R}^{n} \rightarrow \mathbb{R}^{p}$ be a $C^{1}$ semi-algebraic mapping, where $n>p$. Then $S(f) \subseteq \mathcal{N}_{\infty}(f)$.

In particular, from Theorem 2.4.8 and Corollary 2.4.9, we conclude that t-regularity implies $\rho_{E}$-regularity.

Proof. Let $t=\left(t_{1}, \ldots, t_{p}\right) \in S(f)$. Since $\mathcal{M}(f)$ is semi-algebraic, one can use the Curve Selection Lemma (Milnor [34,§3]) to find an analytic path $\left.\phi=\left(\phi_{1}, \ldots, \phi_{n}\right):\right] 0, \epsilon[\rightarrow \mathcal{M}(f) \subset$ $\mathbb{R}^{n}$ such that $\lim _{s \rightarrow 0}\|\phi(s)\|=\infty$ and $\lim _{s \rightarrow 0} f(\phi(s))=t$.

We know that

$$
\phi(s) \in \mathcal{M}(f) \Longleftrightarrow \operatorname{rank}\left(\begin{array}{ccc}
\frac{\partial f_{1}}{\partial x_{1}}(\phi(s)) & \ldots & \frac{\partial f_{1}}{\partial x_{n}}(\phi(s)) \\
\vdots & \vdots & \vdots \\
\frac{\partial f_{p}}{\partial x_{1}}(\phi(s)) & \ldots & \frac{\partial f_{p}}{\partial x_{n}}(\phi(s)) \\
\phi_{1}(s) & \ldots & \phi_{n}(s)
\end{array}\right)<p+1 .
$$

So, there exist curves $\lambda(s), b_{1}(s), \ldots, b_{p}(s)$ such that $\left(\lambda(s), b_{1}(s), \ldots, b_{p}(s)\right) \neq(0, \ldots, 0), \forall s$, and one has the equality:

$$
\lambda(s)\left(\phi_{1}(s), \ldots, \phi_{n}(s)\right)=b_{1}(s) \frac{\partial f_{1}}{\partial x}(\phi(s))+\ldots+b_{p}(s) \frac{\partial f_{p}}{\partial x}(\phi(s)),
$$

where $\frac{\partial f_{i}}{\partial x}(\phi(s))=\left(\frac{\partial f_{i}}{\partial x_{1}}(\phi(s)), \ldots, \frac{\partial f_{i}}{\partial x_{n}}(\phi(s))\right)$, for $i=1, \ldots, p$.

Consider $b(s)=\left(b_{1}(s), \ldots, b_{p}(s)\right)$. From the equality (2.19) and by the statements that $\left(\lambda(s), b_{1}(s), \ldots, b_{p}(s)\right) \neq(0, \ldots, 0), \forall s$, and $\lim _{s \rightarrow 0}\|\phi(s)\|=\infty$, we have $b(s) \neq 0, \forall s$. Consequently, from (2.19), we obtain:

$$
\frac{\lambda(s)}{\|b(s)\|}\left(\phi_{1}(s), \ldots, \phi_{n}(s)\right)=\frac{b_{1}(s)}{\|b(s)\|} \frac{\partial f_{1}}{\partial x}(\phi(s))+\ldots+\frac{b_{p}(s)}{\|b(s)\|} \frac{\partial f_{p}}{\partial x}(\phi(s)) .
$$

We set $\lambda_{0}(s):=\frac{\lambda(s)}{\|b(s)\|}$ and $a(s):=\frac{b(s)}{\|b(s)\|}$. So, $\|a(s)\|=1$ and one obtains the following equalities:

$$
\sum_{i=1}^{p} a_{i}(s) \frac{\mathrm{d}}{\mathrm{d} s} f_{i}(\phi(s))=\left\langle\sum_{i=1}^{p} a_{i}(s) \frac{\partial f_{i}}{\partial x}(\phi(s)), \phi^{\prime}(s)\right\rangle=\frac{1}{2} \lambda_{0}(s) \frac{\mathrm{d}}{\mathrm{ds}}\|\phi(s)\|^{2},
$$

where the later follows from (2.20), i.e., from the equality $\sum_{i=1}^{p} a_{i}(s) \frac{\partial f_{i}}{\partial x}(\phi(s))=\lambda_{0}(s) \phi(s)$.

On the other hand, since $\lim _{s \rightarrow 0} f_{i}(\phi(s))=t_{i}$, it follows that $\operatorname{ord}_{\mathrm{s}}\left(\frac{\mathrm{d}}{\mathrm{ds}} f_{i}(\phi(s))\right) \geq 0$, for 
$i=1, \ldots, p$. This and the equality (2.21) imply:

$$
0 \leq \operatorname{ord}_{\mathrm{s}}\left(\lambda_{0}(s) \frac{\mathrm{d}}{\mathrm{ds}}\|\phi(s)\|^{2}\right)<\operatorname{ord}_{\mathrm{s}}\left(\lambda_{0}(s)\|\phi(s)\|^{2}\right) .
$$

Now, from (2.20) one obtains:

$$
\operatorname{ord}_{\mathrm{s}}\left(\|\phi(s)\|\left\|a_{1}(s) \frac{\partial f_{1}}{\partial x}(\phi(s))+\ldots+a_{p}(s) \frac{\partial f_{p}}{\partial x}(\phi(s))\right\|\right)=\operatorname{ord}_{\mathrm{s}}\left(\left|\lambda_{0}(s)\right|\|\phi(s)\|^{2}\right),
$$

which is positive by (2.22). Therefore, this last equation implies:

$$
\lim _{s \rightarrow 0}\|\phi(s)\|\left\|a_{1}(s) \frac{\partial f_{1}}{\partial x}(\phi(s))+\ldots+a_{p}(s) \frac{\partial f_{p}}{\partial x}(\phi(s))\right\|=0,
$$

which, in turn, implies $\lim _{s \rightarrow 0}\|\phi(s)\| \nu(\mathrm{D} f(\phi(s)))=0$. This shows that $t \in \mathcal{N}_{\infty}(f)$.

The converse of Proposition 2.5.4 and Proposition are not true in general, as the next example shows:

EXAmple 2.5.6 (Păunescu and Zaharia [45, Proposition 1.8]). The polynomials $f_{n, q}: \mathbb{K}^{3} \rightarrow \mathbb{K}$, $f_{n, q}\left(x_{1}, x_{2}, x_{3}\right):=x_{1}-3 x_{1}^{2 n+1} x_{2}^{2 q}+2 x_{1}^{3 n+1} x_{2}^{3 q}+x_{2} x_{3}$, where $n, q \in \mathbb{N} \backslash\{0\}$, are $\rho_{E}$-regular at infinity, more precisely $S\left(f_{n . q}\right)=\emptyset$. It is shown in [45] that $f_{n, q}$ satisfies Malgrange condition (hence it is $t$-regular at infinity) for any $t \in \mathbb{K}$ if and only if $n \leq q$. For $n>q$ we therefore get $\emptyset=S\left(f_{n . q}\right) \subsetneq \mathcal{N}_{\infty}\left(f_{n . q}\right) \neq \emptyset$. In particular, for $n>q$ the polynomial is $\rho_{E^{-r e g u l a r}}$ but not $t$-regular. 


\section{Asymptotic theorems for $\rho_{E}$-regularity}

\section{Contents}

3.1 Morse-Sard type theorem for $\rho_{E}$-regular values $\ldots \ldots \ldots \ldots$

3.1.1 Partial Thom stratification at infinity . . . . . . . . . . . 41

3.1 .2 Proof of Theorem $3.1 .1 \ldots \ldots \ldots \ldots$. . . . . . . . . 42

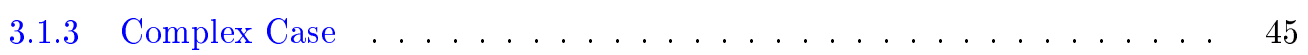

3.2 Diagram ...................... 46

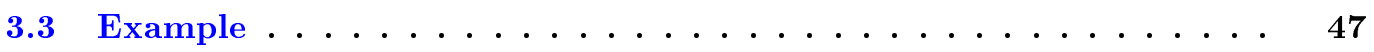

\section{Brief Resume}

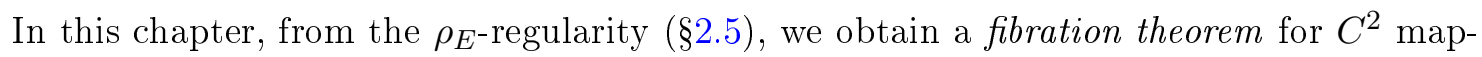
pings $f: X \rightarrow \mathbb{R}^{p}$, where $X \subset \mathbb{R}^{n}$ is a submanifold (Theorem 3.1.1 (a)). In the case that $f$ is a $C^{1}$ semi-algebraic mapping and $X$ a semi-algebraic set, we obtain a structure theorem for the sets $S(f)$ and $A_{\rho_{E}}(f)$ (Theorem 3.1.1 (b)). These results are based on the existence of partial Thom stratifications at infinity, cf Definition 3.1.3, and on a fibration theorem "at infinity", Proposition 3.1.5. The case of polynomial mappings $f: \mathbb{C}^{n} \rightarrow \mathbb{C}^{p}$ will be discussed in 3.1.3.

The above theorems represent an asymptotic Morse-Sard type theorem for the $\rho_{E^{-}}$ regularity and they refine Theorem 2.3.5 (fibration theorem due to Rabier [48]) and Theorem 2.3.6 (structure theorem due to Kurdyka, Orro, Simon [29]). In fact, the regularity conditions used in Theorem 2.3.5 and Theorem 2.3.6 are superseded by the $\rho_{E}$-regularity, which is a more general condition, cf. Proposition 2.5.4, Proposition 2.5.5 and Example 2.5.6.

We present in $\S 3.2$ a diagram that summarises the main results of this chapter and Chapter 2 in a condensed manner and to finish, we discuss an example of a family of polynomial functions in $\S 3.3$.

The main results of this chapter have been published in [11]. 


\subsection{Morse-Sard type theorem for $\rho_{E}$-regular values}

The main purpose of this chapter is the following (the notations are as in $§ 2.5$ ):

Theorem 3.1.1 (Asymptotic Morse-Sard type theorem for $\rho_{E}$-regularity).

Let $f: X \rightarrow \mathbb{R}^{p}$ be a $C^{2}$ mapping on a submanifold $X \subset \mathbb{R}^{n}$, where $\operatorname{dim} X>p>0$. Then:

(a) (Fibration theorem).

$S(f)$ is a closed set. Moreover, if $X$ is closed, then $A_{\rho_{E}}:=f(\operatorname{Sing} f) \cup S(f)$ is a closed set and the restriction:

$$
f_{\mid}: X \backslash f^{-1}\left(A_{\rho_{E}}\right) \rightarrow \mathbb{R}^{p} \backslash A_{\rho_{E}}
$$

is a locally trivial fibration over each connected component of $\mathbb{R}^{p} \backslash A_{\rho_{E}}$.

In particular $B(f) \subset A_{\rho_{E}}$.

(b) (Structure theorem).

Assume that $X$ is a semi-algebraic set and that $f$ is a semi-algebraic mapping.

Then $S(f)$ and $A_{\rho_{E}}$ are semi-algebraic sets of dimension $\leq p-1$.

The proof of above theorem will be presented in §3.1.2. Here, we just discuss some consequences of this result and present the structure of this chapter.

Let $f: \mathbb{R}^{n} \rightarrow \mathbb{R}^{p}$ be a $C^{2}$ semi-algebraic mapping. Theorem 3.1.1 means a Morse-Sard type theorem for $\rho_{E}$-regularity in the context of semi-algebraic mappings. In fact, from (a) and (b), we have that $A_{\rho_{E}}$ is a closed semi-algebraic set of dimension at most $p-1$, which implies that $A_{\rho_{E}}$ has volume zero and therefore $\mathbb{R}^{p} \backslash A_{\rho_{E}}$ is a dense open set in $\mathbb{R}^{p}$. This means that (from item (a)) a non-proper semi-algebraic mapping $f$ is a fibration on each connected component of the dense open set $\mathbb{R}^{p} \backslash A_{\rho_{E}}$.

In fact, we can say more about the structure of the set $\mathbb{R}^{p} \backslash A_{\rho_{E}}$ in the setting of semialgebraic mappings $f$. We have that $\mathbb{R}^{p} \backslash A_{\rho_{E}}$ is semi-algebraic since $A_{\rho_{E}}$ is semi-algebraic and consequently $\mathbb{R}^{p} \backslash A_{\rho_{E}}$ has a finite number of connected component (every semi-algebraic set has a finite number of connected components, see [3, Theorem 2.4.5]). Therefore, directly from Theorem 3.1.1 we have that outside a semi-algebraic set of dimension $\leq p-1$, the fibres of $f$ have a finite number of topological types.

For $f$ as above and from Theorem 2.4.8, Corollary 2.4.9, Proposition 2.5.4 and Proposition 2.5.5, we have the following inclusion and equality: $S(f) \subset \mathcal{N}_{\infty}(f)=\mathcal{N} \mathcal{T}_{\infty}(f)$, which inclusion can be strict, cf. Example 2.5.6. Therefore, Theorem 3.1.1 refines Theorem 2.3.5 and Theorem 2.3.6 for semi-algebraic mappings in the way that the key set $\mathcal{N}_{\infty}(f)$ of Theorem 2.3.5 and Theorem 2.3.6 is substituted by $S(f)$.

Let us point out that the proof of Theorem 3.1.1 has a completely different flavour than the proofs of Theorem 2.3.5 and Theorem 2.3.6. The proof of item (b) is based on the existence of a partial Thom stratification at infinity, cf Definition 3.1.3 and Propostion 3.1.2; the item (a) is based on Proposition 3.1.5, which is a fibration theorem "at infinity". 
This chapter is organised as follows: in $§ 3.1 .1$, we define the partial Thom stratification at infinity and show that there exists a partial Thom stratification at infinity in the setting of semi-algebraic mappings. In $\S 3.1 .2$, we give the proof of Theorem 3.1.1.

In $\S 3.2$ we present a diagram that summarises the main results of this chapter and Chapter 2 in a condensed manner. In $\S 3.3$, we give an example of a family of polynomial functions and discuss its properties.

The notations of this chapter are the same as in subsection "Notation (page 27)". We use coordinates $\left(x_{1}, \ldots, x_{n}\right)$ for the affine space $\mathbb{K}^{n}$, coordinates $\left[x_{0}: x_{1}: \ldots: x_{n}\right]$ for the projective space $\mathbb{P}^{n}$ and we consider the canonical embedding of $\mathbb{K}^{n}$ in $\mathbb{P}^{n}\left(\left(x_{1}, \ldots, x_{n}\right) \mapsto[1\right.$ : $\left.\left.x_{1}: \ldots: x_{n}\right]\right)$. We set $\mathbb{H}^{\infty}:=\left\{\left[x_{0}: \ldots: x_{n}\right] \in \mathbb{P}^{n} \mid x_{0}=0\right\}$ the hyperplane at infinity.

Given a $C^{1}$ mapping $f: X \rightarrow \mathbb{K}^{p}$, where $X \subset \mathbb{K}^{n}$ is submanifold, we set $\mathbb{X}:=\overline{\operatorname{graph} f}$ the closure of the graph of $f$ in $\mathbb{P}^{n} \times \mathbb{K}^{p}$ (via the embedding of $\mathbb{K}^{n}$ in $\mathbb{P}^{n}$ ), we set $\mathbb{X}^{\infty}:=$ $\mathbb{X} \cap\left(\mathbb{H}^{\infty} \times \mathbb{K}^{p}\right)$ and we denote by $\tau: \mathbb{P}^{n} \times \mathbb{K}^{p} \rightarrow \mathbb{K}^{p}$ the second projection.

With these notations we have:

\subsubsection{Partial Thom stratification at infinity}

We show that $\mathbb{X}^{\infty}$ may be endowed with a stratification having good enough properties such that one may use it to define the stratified singular locus of $\tau_{\mid \mathbb{X}^{\infty}}$. By "stratifications" we mean, as usual, locally finite stratifications satisfying the frontier condition. For some strata $\mathcal{S}_{1}, \mathcal{S}_{2}$, we write $\mathcal{S}_{2} \prec \mathcal{S}_{1}$ to say that $\mathcal{S}_{2} \subset \overline{\mathcal{S}_{1}} \backslash \mathcal{S}_{1}$.

Proposition 3.1.2. Let $f: X \rightarrow \mathbb{K}^{p}$ be a semi-algebraic $C^{1}$-mapping on a smooth semialgebraic subset $X \subset \mathbb{K}^{n}$ for $\mathbb{K}=\mathbb{R}$ or $f: X \rightarrow \mathbb{K}^{p}$ be a restriction of a polynomial mapping on a smooth algebraic set $X \subset \mathbb{C}^{n}$. There exists a semi-algebraic Whitney (a)-regular stratification $\mathcal{S}$ (for $\mathbb{K}=\mathbb{C}$, the closure of each stratum of $\mathcal{S}$ is an algebraic set) of $\mathbb{X}$ such that $\mathbb{X}^{\infty}$ is a union of strata, and that any pair of strata $B \prec A$, with $A \subset \mathbb{X} \backslash \mathbb{X}^{\infty}$ and $B \subset \mathbb{X}^{\infty}$, satisfy the Thom $\left(a_{g}\right)$-regularity condition with respect to some function $g$ defining locally $\mathbb{X}^{\infty}$ in $\mathbb{X}$.

Proof. We follow $[54, \S 2]$ and start with some Whitney (a)-regular stratification of $\mathbb{X}$ with semi-algebraic strata (for $\mathbb{K}=\mathbb{C}$, the closure of each stratum is an algebraic set, Whitney [59, page 540]); this exists after Whitney [59], see also [12, Chapter I] and [20, Chapter I]. One then refines it to a semi-algebraic stratification such that $\mathbb{X}^{\infty}$ is a union of strata (for $\mathbb{K}=\mathbb{C}$, to a stratification such that the closure of each stratum is an algebraic set and $\mathbb{X}^{\infty}$ is a union of strata), see [12, page 6], [20, Chapter I] or [59, Theorem 19.2]. Next, since the $\left(a_{g}\right)$-regularity condition is stratifiable (see e.g. [1, §3], [10], [20, Chapter I]), applying the Thom condition to the pairs of strata as in the above statement yields a further refinement which is the desired stratification $\mathcal{S}$, at least locally. 
However we need to show that this refinement is a globally defined stratification of $\mathbb{X}^{\infty}$. The argument goes as follows: in the $\left(a_{g}\right)$-regularity test we take the limits at some point of $\mathbb{X}^{\infty}$ of the tangent hyperplanes along strata coming from $\mathbb{X} \backslash \mathbb{X}^{\infty}$. These limits are precisely described by the space of characteristic covectors at infinity $\mathcal{C}^{\infty}$. But by Lemma $2.4 .4, \mathcal{C}^{\infty}$ is independent of the function $g$ defining $\mathbb{X}^{\infty}$ locally.

Definition 3.1.3. We call partial Thom stratification at infinity a stratification $\mathcal{S}$ as in Proposition 3.1.2.

Such stratification has been introduced in [54, Definition 2.1] (see also [56, Appendix 1]), for polynomial functions $f: \mathbb{K}^{n} \rightarrow \mathbb{K}$ and depends of course on the embedding $X \subset \mathbb{K}^{n}$.

\subsubsection{Proof of Theorem 3.1.1}

\section{Proof of (b)}

The image $f(\operatorname{Sing} f)$ by $f$ of the semi-algebraic set $\operatorname{Sing} f$ is semi-algebraic, by TarskiSeidenberg theorem (see also [3, Proposition 2.2.7]), and of dimension $\leq p-1$ by the semialgebraic Sard theorem.

To show that $S(f)$ is semi-algebraic, we use the semi-algebraic embedding $\varphi: \mathbb{R}^{n} \rightarrow \mathbb{R}^{n+1} \times$ $\mathbb{R}^{p}, x=\left(x_{1}, \ldots, x_{n}\right) \mapsto\left(\frac{1}{\sqrt{1+\|x\|^{2}}}, \frac{x_{1}}{\sqrt{1+\|x\|^{2}}}, \ldots, \frac{x_{n}}{\sqrt{1+\|x\|^{2}}}, f(x)\right)$. Let $V_{1}:=\overline{\varphi(\mathcal{M}(f))} \cap$ $\left\{\left(z_{0}, z_{1}, \ldots, z_{n}, t\right) \in \mathbb{R}^{n+1} \times \mathbb{R}^{p} \mid z_{0}=0\right\}$ and let $\pi: \mathbb{R}^{n+1} \times \mathbb{R}^{p} \rightarrow \mathbb{R}^{p}$ be the canonical projection. Then $V_{1}$ is semi-algebraic and $S(f)=\pi\left(V_{1}\right)$, so we may conclude by the TarskiSeidenberg theorem that $S(f)$ is semi-algebraic.

To prove the dimension assertion for $S(f)$ we follow the notations and definitions of §3.1.1. Let $\mathcal{S}=\left\{\mathcal{S}_{i}\right\}_{i \in I}$ be a semi-algebraic partial Thom stratification at infinity, the existence of which has been proved above. Consider the projection $\tau: \mathbb{P}^{n} \times \mathbb{R}^{p} \rightarrow \mathbb{R}^{p}$ and $t_{0} \in \mathbb{R}^{p}$. The critical locus at infinity of the restriction $\tau_{\mid \mathbb{X}}$ with respect to $\mathcal{S}$ is defined as follows:

$$
\operatorname{Sing}_{\mathcal{S}}^{\infty} \tau_{\mid \mathbb{X}}:=\bigcup_{\mathcal{S}_{i} \subset \mathbb{X} \infty} \operatorname{Sing} \tau_{\mid \mathcal{S}_{i}}
$$

Since the stratification of $\mathbb{X}^{\infty}$ is in particular Whitney (a)-regular, it follows that $\operatorname{Sing}_{\mathcal{S}}^{\infty} \tau_{\mathbb{X}}$ is a closed semi-algebraic subset of $\mathbb{X}^{\infty}$. Then, by the semi-algebraic Sard theorem, the image $\tau\left(\operatorname{Sing}_{\mathcal{S}}^{\infty} \tau_{\mid \mathbb{X}}\right) \subset \mathbb{R}^{p}$ is semi-algebraic and of dimension $\leq p-1$.

On the other hand, it also follows from the definition of $t$-regularity that:

$$
z_{0} \notin \operatorname{Sing}_{\mathcal{S}}^{\infty} \tau_{\mid \mathbb{X}} \Longrightarrow f \text { is } t \text {-regular at } z_{0},
$$

which implies that $\mathbb{R}^{p} \backslash \tau\left(\operatorname{Sing}_{\mathcal{S}}^{\infty} \tau_{\mid \mathbb{X}}\right)$ is included in the set of $t$-regular values of $f$. Therefore, by Proposition 2.5.4 (or Proposition 2.5.5), we get the inclusion $\mathbb{R}^{p} \backslash \tau\left(\operatorname{Sing}_{\mathcal{S}}^{\infty} \tau_{\mid \mathbb{X}}\right) \subset \mathbb{R}^{p} \backslash S(f)$, which shows that $\operatorname{dim} S(f) \leq p-1$. 


\section{Proof of (a)}

Let $t_{0} \in \overline{S(f)}$ and let $\left\{t_{i}\right\}_{i \in \mathbb{N}} \subset S(f)$ be a sequence such that $\lim _{i \rightarrow \infty} t_{i}=t_{0}$. By definition, for every $t_{i}$, we have a sequence $\left\{x_{i, k}\right\}_{k \in \mathbb{N}} \subset \mathcal{M}(f)$ such that $\lim _{k \rightarrow \infty}\left\|x_{i, k}\right\|=\infty$ and $\lim _{k \rightarrow \infty} f\left(x_{i, k}\right)=t_{i}$. Thus, for each $i$, there exists $k(i) \in \mathbb{N}$ such that if $k \geqslant k(i)$ then $\left\|x_{i, k}\right\|>i$ and $\left|f\left(x_{i, k}\right)-t_{i}\right|<1 / i$. Setting $x_{i}:=x_{i, k(i)}$, one gets a sequence $\left\{x_{i}\right\}_{i \in \mathbb{N}} \subset \mathcal{M}(f)$ such that $\lim _{i \rightarrow \infty}\left\|x_{i}\right\|=\infty$ and $\lim _{i \rightarrow \infty} f\left(x_{i}\right)=t_{0}$. This shows that $t_{0} \in S(f)$, hence $S(f)$ is closed.

Let us assume now that $X$ is closed and let $t_{0} \in \overline{f(\operatorname{Sing} f) \cup S(f)}$. Note that, by basic arguments of topology, we have $\overline{f(\operatorname{Sing} f) \cup S(f)}=\overline{f(\operatorname{Sing} f)} \cup \overline{S(f)}$. Thus, we may assume that $t_{0} \in \overline{f(\operatorname{Sing} f)}$ since we have just proved that $S(f)$ is closed. Then there exists a sequence $\left\{x_{j}\right\}_{j \in \mathbb{N}} \subset \operatorname{Sing} f$, such that $\lim _{j \rightarrow \infty} f\left(x_{j}\right)=t_{0}$. If $\left\{x_{j}\right\}_{j \in \mathbb{N}}$ is non-bounded, we may choose a subsequence $\left\{x_{j_{k}}\right\}_{k \in \mathbb{N}}$ such that $\lim _{k \rightarrow \infty}\left\|x_{j_{k}}\right\|=\infty$ and $\lim _{k \rightarrow \infty} f\left(x_{j_{k}}\right)=t_{0}$. Since $\operatorname{Sing} f \subset$ $\mathcal{M}(f)$, it follows that $t_{0} \in S(f)$ which is closed. If $\left\{x_{j}\right\}_{j \in \mathbb{N}}$ is bounded, then we may choose a subsequence $\left\{x_{j_{k}}\right\}_{k \in \mathbb{N}}$ such that $\lim _{k \rightarrow \infty} x_{j_{k}}=x_{0} \in X$ since $X$ is assumed to be closed, and that $\lim _{k \rightarrow \infty} f\left(x_{j_{k}}\right)=t_{0}$. Since $\operatorname{Sing} f$ is a closed set, this implies $x_{0} \in \operatorname{Sing} f$, and we get $t_{0}=f\left(x_{0}\right) \in f(\operatorname{Sing} f)$, which shows that $t_{0} \in f(\operatorname{Sing} f) \cup S(f)$.

Let us finally show the fibration statement. We first prove a fibration result in the neighbourhood of infinity.

Definition 3.1.4 (Topological triviality at infinity). We say that $f$ is topologically trivial at infinity at the value $t_{0} \in \mathbb{R}^{p}$ if there exists a compact set $K \subset \mathbb{R}^{n}$ and a ball $B_{\delta} \subset \mathbb{R}^{p}$ centred at $t_{0}$ such that the restriction:

$$
f_{\mid}:(X \backslash K) \cap f^{-1}\left(B_{\delta}\right) \rightarrow B_{\delta}
$$

is a trivial topological fibration.

Note that one may have two situations in which the mapping (3.1) may be a topologically trivial fibration at infinity, namely whenever $B_{\delta} \subset \operatorname{Im} f$ or when $B_{\delta} \subset \mathbb{R}^{p} \backslash \operatorname{Im} f$. Otherwise (i.e. $B_{\delta} \cap \operatorname{Im} f \neq \emptyset$ and $\left.B_{\delta} \cap\left(\mathbb{R}^{p} \backslash \operatorname{Im} f\right) \neq \emptyset\right) f$ cannot be a fibration since one has simultaneously empty fibre and non-empty fibre.

Proposition 3.1.5 ( $\rho$-regularity implies topological triviality at infinity). Let $f: X \rightarrow \mathbb{R}^{p}$ be a $C^{2}$ mapping, for $n>p$. If the fibre $f^{-1}\left(t_{0}\right)$ is $\rho$-regular at infinity, then $f$ is topologically trivial at infinity at $t_{0}$.

In particular, $f$ is topologically trivial at infinity at any value of $\mathbb{R}^{p} \backslash S(f)$.

Proof. Let $t_{0} \notin S(f)$. Since $S(f)$ is a closed set, there exists a closed ball $D$ centred at $t_{0}$ and included in $\mathbb{R}^{p} \backslash S(f)$. Then there exists some large enough radius $R_{0} \gg 0$ such that:

$$
\mathcal{M}(f) \cap f^{-1}(D) \backslash B_{R_{0}}^{n}=\emptyset .
$$


Indeed, if this were not true, then there exists a sequence $\left\{x_{k}\right\}_{k \in \mathbb{N}} \subset f^{-1}(D) \cap \mathcal{M}(f)$ with $\lim _{k \rightarrow \infty}\left\|x_{k}\right\|=\infty$ and since $D$ is compact, one may extract a sub-sequence $\left\{x_{k_{i}}\right\}_{i \in \mathbb{N}} \subset \mathcal{M}(f)$ with $\lim _{k \rightarrow \infty} f\left(x_{k_{i}}\right)=t \in D$, which gives a contradiction to $D \cap S(f)=\emptyset$.

To prove the topological triviality at infinity at $t_{0}$ it is enough to show that the mapping:

$$
f_{\mid}: f^{-1}(D) \backslash B_{R}^{n} \rightarrow D
$$

is a trivial fibration on the manifold with boundary $\left(f^{-1}(D) \backslash B_{R}^{n}, f^{-1}(D) \cap S_{R}^{n-1}\right)$, for any $R \geq R_{0}$. This is a submersion by (3.2) but it is not proper, so one cannot apply Ehresmann's theorem directly. Instead, we consider the map $(f, \rho): f^{-1}(D) \backslash B_{R}^{n} \rightarrow D \times[R, \infty[$. Now, as a direct consequence of its definition, this is a proper map. It is still a submersion by (3.2) and since $\operatorname{Sing}(f, \rho)=\mathcal{M}(f)$. We then apply Ehresmann's theorem to the mapping $(f, \rho)$ in order to conclude that it is a locally trivial fibration, hence trivial over $D \times[R, \infty[$. Take now the projection $\pi: D \times[R, \infty[\rightarrow D$ which is a trivial fibration by definition and remark that our map (3.3) is the composition $\pi \circ(f, \rho)$ of two trivial fibrations, hence a trivial fibration too.

REMARK 3.1.6. The implication in the above proposition is not an equivalence in general, see for instance Example 3.3.1. It is easier to give such examples in the topological category, for instance $f(x, y)=x^{3}$ which is topologically equivalent to the projection on $x$, whereas $S(f)=\{0\}$.

We now complete the proof of Theorem 3.1.1, item (a). Since $\mathbb{R}^{p} \backslash(f(\operatorname{Sing} f) \cup S(f))$ is an open set, for any fixed $t_{0} \notin f(\operatorname{Sing} f) \cup S(f)$ there exists a closed ball $D$ centred at $t_{0}$ such that $D \subset \mathbb{R}^{p} \backslash f(\operatorname{Sing} f) \cup S(f)$. By the above proof of Proposition 3.1.5 and using the same notations, one has the trivial fibration (3.3) on the manifold with boundary $\left(f^{-1}(D) \backslash B_{R}^{n}, f^{-1}(D) \cap S_{R}^{n-1}\right)$, for any $R \geq R_{0}$.

Next, since $D \cap f(\operatorname{Sing} f)=\emptyset$, the restriction:

$$
f_{\mid}: f^{-1}(D) \cap \bar{B}_{R_{0}}^{n} \rightarrow D
$$

is a proper submersion on the manifold with boundary $\left(f^{-1}(D) \cap \bar{B}_{R_{0}}^{n}, f^{-1}(D) \cap S_{R_{0}}^{n-1}\right)$ and therefore a locally trivial fibration by Ehresmann's theorem, hence a trivial fibration over $D$.

We finally glue together the two trivial fibrations (3.4) and (3.3) by using an isotopy and the trivial fibration from the following commutative diagram, for some $R>R_{0}$ :

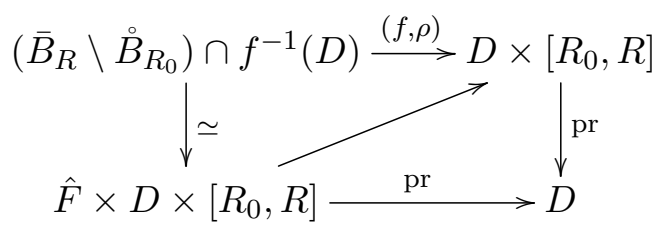

where $\hat{F}$ denotes the fibre of the trivial fibration $f_{\mid}: S_{R} \cap f^{-1}(D) \rightarrow D$ and does not depend 
on the radius $R>R_{0}$.

REMARK 3.1.7. If in item (a) of Theorem 3.1.1, we do not assume that $X$ is closed then the fibration assertion holds if one replaces in the statement $f(\operatorname{Sing} f)$ by its closure $\overline{f(\operatorname{Sing} f)}$.

\subsubsection{Complex Case}

Let $f: \mathbb{C}^{n} \rightarrow \mathbb{C}^{p}$ be a polynomial mapping. Directly from Theorem 3.1.1, we obtain that the restriction:

$$
f_{\mid}: \mathbb{C}^{n} \backslash f^{-1}\left(A_{\rho_{E}}\right) \rightarrow \mathbb{C}^{p} \backslash A_{\rho_{E}}
$$

is a locally trivial fibration over $\mathbb{C}^{p} \backslash A_{\rho_{E}}$ and that $S(f)$ and $A_{\rho_{E}}$ are real semi-algebraic sets of real dimension $\leq 2 p-1$.

In this subsection, we refine this dimension statement by showing that $S(f)$ and $A_{\rho_{E}}$ are real semi-algebraic sets of real dimension $\leq 2 p-2$ (Theorem 3.1.8).

This can be obtained as a consequence of Propositions 2.5.4, 2.5.5 and Theorem 2.3.6 (due to Kurdyka, Orro and Simon [29]). In fact, from these two propositions, one has $A_{\rho_{E}}, S(f) \subset$ $\mathcal{N}(f):=\left(f(\operatorname{Sing} f) \cup \mathcal{N}_{\infty}(f)\right)$ and, since $\mathcal{N}(f)$ is a complex algebraic set of dimension $\leq p-1$, $A_{\rho_{E}}$ and $S(f)$ are real semi-algebraic sets (these follow, respectively, from Theorem 2.3.6 and Theorem 3.1.1), we obtain that $A_{\rho_{E}}$ and $S(f)$ are real semi-algebraic sets of real dimension $\leq 2 p-2$.

However, in Theorem 3.1.8, we present a proof which does not depend of Kurdyka, Orro and Simon's result.

Theorem 3.1.8 (Asymptotic Morse-Sard type theorem for the $\rho_{E^{-}}$-regularity in the complex case). Let $f: \mathbb{C}^{n} \rightarrow \mathbb{C}^{p}$ be a polynomial mapping, where $n>p$. Then $S(f)$ and $A_{\rho_{E}}:=f(\operatorname{Sing} f) \cup S(f)$ are closed real semi-algebraic sets of real dimension $\leq 2 p-2$ and the restriction:

$$
f_{\mid}: \mathbb{C}^{n} \backslash f^{-1}\left(A_{\rho_{E}}\right) \rightarrow \mathbb{C}^{p} \backslash A_{\rho_{E}}
$$

is a locally trivial fibration over $\mathbb{C}^{p} \backslash A_{\rho_{E}}$. In particular $B(f) \subset A_{\rho_{E}}$.

Proof. As we have said before, the proof that $S(f)$ and $A_{\rho_{E}}$ are closed sets and the fibration result follow as in proof of Theorem 3.1.1. The proof that $S(f)$ and $A_{\rho_{E}}$ are real semi-algebraic sets follow as in proof of Theorem 3.1.1 (b) (page 42) with the same function $\varphi$ used in that proof.

We concentrate on the dimension statement. We use notations and definitions of §3.1.1. Let $\mathscr{S}=\left\{\mathcal{S}_{i}\right\}_{i \in I}$ be a partial Thom stratification at infinity of $\mathbb{X}$ (the existence of this stratification has been obtained in Proposition 3.1.2) and the projection $\tau: \mathbb{P}^{n} \times \mathbb{C}^{p} \rightarrow \mathbb{C}^{p}$. The critical locus at infinity of the restriction $\tau_{\mid \mathbb{X}}$ with respect to $\mathscr{S}$ is defined as follows:

$$
\operatorname{Sing}_{\mathscr{S}}^{\infty} \tau_{\mid \mathbb{X}}:=\bigcup_{\mathcal{S}_{i} \subset \mathbb{X}^{\infty}} \operatorname{Sing} \tau_{\mid \mathcal{S}_{i}}
$$


Since the stratification of $\mathbb{X}^{\infty}$ is in particular Whitney (a)-regular, the closure of each stratum $\mathcal{S}_{i} \subset \mathbb{X}^{\infty}$ is an algebraic set (Whitney [59, page 540]), which implies that $\operatorname{Sing}_{\mathscr{S}}^{\infty} \tau_{\mid \mathbb{X}}$ is an algebraic subset of $\mathbb{X}^{\infty}$. Then, by Chevalley's theorem, the image $\tau\left(\operatorname{Sing}_{\mathscr{S}}^{\infty} \tau_{\mathbb{X}}\right) \subset \mathbb{C}^{p}$ is a constructible set (see e.g. Mumford [37, page 51]) and, moreover, $\overline{\tau\left(\operatorname{Sing}_{\mathscr{S}}^{\infty} \tau_{\mid \mathbb{X}}\right)} \subset \mathbb{C}^{p}$ is an algebraic set (closed constructible sets are algebraic sets, see e.g. Mumford [37, page 57 and Corollary 1 (page 60)] or Milne [33, page 215 and Proposition 15.2]). Then, it follows from Sard's theorem and second statement of Mumford [36, Proposition 2.31] that $\overline{\tau\left(\operatorname{Sing}_{\mathscr{S}}^{\infty} \tau_{\mid \mathbb{X}}\right)}$ is a complex algebraic set of complex dimension $\leq p-1$.

On the other hand, it also follows from the definition of $t$-regularity that:

$z_{0} \notin \operatorname{Sing}_{\mathscr{S}}^{\infty} \tau_{\mid \mathbb{X}} \Longrightarrow f$ is $t$-regular at $z_{0}$

which implies that $\mathbb{C}^{p} \backslash \overline{\tau\left(\operatorname{Sing}_{\mathscr{S}}^{\infty} \tau_{\mid \mathbb{X}}\right)}$ is included in the set of $t$-regular values of $f$. Therefore, by Proposition 2.5.4 (or Proposition 2.5.5), we get the inclusion $\mathbb{C}^{p} \backslash \overline{\tau\left(\operatorname{Sing}_{\mathscr{S}}^{\infty} \tau_{\mid \mathbb{X}}\right)} \subset \mathbb{C}^{p} \backslash S(f)$, which shows that the real dimension of $S(f)$ is at most $2 p-2$.

Now, since $\operatorname{Sing} f$ is an algebraic set and $f$ is a polynomial mapping, we have that $f(\operatorname{Sing} f)$ is a constructible set (Chevalley's theorem, see e.g. Mumford [37, page 51]) and $\overline{f(\operatorname{Sing} f)} \subset \mathbb{C}^{p}$ is an algebraic set (closed constructible sets are algebraic sets, see e.g. Mumford [37, page 57 and Corollary 1 (page 60)] or Milne [33, page 215 and Proposition 15.2]). Then, it follows from Sard's theorem and second statement of Mumford [36, Proposition 2.31] that $\overline{f(\operatorname{Sing} f)}$ is a complex algebraic set of complex dimension $\leq p-1$. This and the dimension statement of $S(f)$ imply that $A_{\rho_{E}}=f(\operatorname{Sing} f) \cup S(f)$ has real dimension at most $2 p-2$, which ends the proof.

In the following example we show that $f(\operatorname{Sing} f)$ is not closed in general.

Example 3.1.9. Consider $f: \mathbb{K}^{2} \rightarrow \mathbb{K}^{2}, f\left(x_{1}, x_{2}\right)=\left(\left(x_{1} x_{2}-1\right)^{2}, x_{2}\right)$. Then Sing $f=$ $\left\{\left(x_{1}, x_{2}\right) \mid x_{2}\left(x_{1} x_{2}-1\right)=0\right\}$ and $f(\operatorname{Sing} f)=\left(\{(1,0)\} \cup\left\{\left(0, x_{2}\right) \mid x_{2} \neq 0\right\}\right)$, which it is not closed.

\subsection{Diagram}

Let $f: \mathbb{K}^{n} \rightarrow \mathbb{K}^{p}$ be a $C^{2}$ semi-algebraic mapping for $\mathbb{K}=\mathbb{R}$ and a polynomial mapping for $\mathbb{K}=\mathbb{C}$. We have the following diagram: 
Figure 3.1: Synopsis.

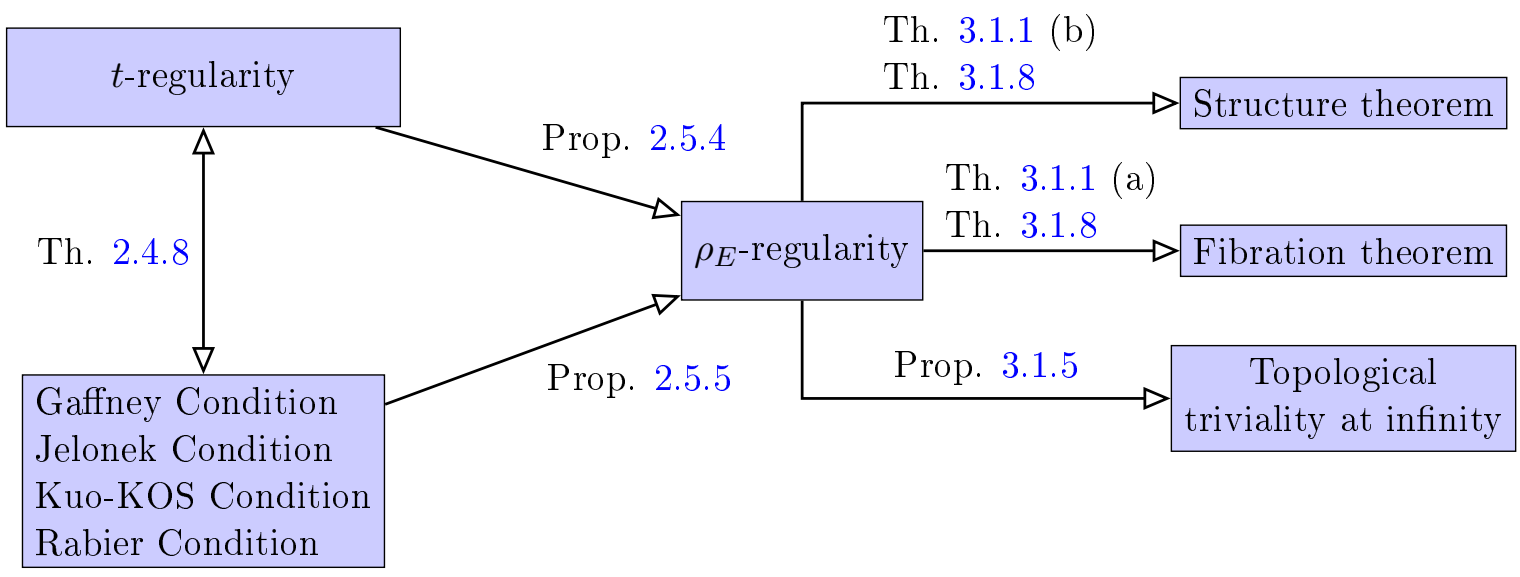

\subsection{Example}

Tibăr and Zaharia present in [57, Example 3.4] an explicit polynomial $f: \mathbb{R}^{2} \rightarrow \mathbb{R}$, constructed with the help of Henry King, such that $0 \in S(f)$ (consequently $0 \in \mathcal{N}_{\infty}(f)^{1}$ by Proposition 2.5.4 and Proposition 2.5.5), but $f$ is a $C^{\infty}$ trivial fibration, which shows, in particular, that $B(f)=\emptyset$. We call this example by King-Tibăr-Zaharia's example. This example is interesting because this phenomenon ( $f$ a $C^{\infty}$ trivial fibration and $S(f) \neq \emptyset$ ) does not occur in the case of complex polynomial functions $f: \mathbb{C}^{2} \rightarrow \mathbb{C}$. In fact, for complex polynomial functions $f: \mathbb{C}^{2} \rightarrow \mathbb{C}$, we know that $f$ is a $C^{\infty}$ trivial fibration on a neighbourhood of $t_{0}$ if and only if $t_{0} \notin \mathcal{N}_{\infty}(f)$ (for references about this last equivalence, see for instance Parusiński [42, Theorem 1.4], Siersma and Tibăr [49, pages 781 and 782]).

This type of examples also shows that the implication in the Proposition 3.1.5 is not an equivalence in general.

Inspired by King-Tibăr-Zaharia's example, we present in this section a family of polynomial functions $f_{a, b, c}: \mathbb{R}^{2} \rightarrow \mathbb{R}$, where $a, b, c \in \mathbb{R}$ are the parameters of this family, and discuss the behaviour of the elements of this family. In particular, we remark that this family contains the King-Tibăr-Zaharia's example (see Remark 3.3.2).

EXAMPLE 3.3.1. Let $f_{a, b, c}: \mathbb{R}^{2} \rightarrow \mathbb{R}$ be a family of polynomial functions with parameters $a, b, c \in \mathbb{R}$, where $f_{a, b, c}(x, y)=y\left(a x^{2} y^{2}+b x y+c\right)$. Then:

(i) If $b^{2}-4 a c<0$, then $f_{a, b, c}$ is a submersion, a $C^{\infty}$ fibration and $B\left(f_{a, b, c}\right)=\emptyset$.

(ii) If $b^{2}-4 a c<0$ and $0<b^{2}-3 a c$, then $f_{a, b, c}$ is a submersion, a $C^{\infty}$ fibration, $B\left(f_{a, b, c}\right)=\emptyset$ and $0 \in S\left(f_{a, b, c}\right)$. Therefore, the polynomials $f_{a, b, c}$ with $b^{2}-4 a c<0$ and $0<b^{2}-3 a c$, are examples like the King-Tibăr-Zaharia's example. We also remark here that the

\footnotetext{
${ }^{1}$ see Definition 2.3.1 and Remark 2.3.4 for the definition of $\mathcal{N}_{\infty}(f)$ in the case of polynomial function.
} 
conditions $b^{2}-4 a c<0$ and $0<b^{2}-3 a c$ are clearly open conditions on the set of polynomial functions $f: \mathbb{R}^{2} \rightarrow \mathbb{R}$.

(iii) If $b^{2}-4 a c<0$ and $0 \geq b^{2}-3 a c$, then $f_{a, b, c}$ is submersion, a $C^{\infty}$ fibration, $B\left(f_{a, b, c}\right)=\emptyset$ and $S\left(f_{a, b, c}\right)=\emptyset$.

(iv) If $b^{2}=4 a c$ then we have the following four cases: $f_{a, b, c}=0, f_{a, b, c}=a x^{2} y^{3}$ (with $a \neq 0$ ), $f_{a, b, c}=c y($ with $c \neq 0)$ and $f_{a, b, c}=a y\left(x y+\frac{b}{2 a}\right)^{2}($ with $a \neq 0, b \neq 0$ and $c \neq 0)$. The first three cases are easy to study and in the case $f_{a, b, c}=a y\left(x y+\frac{b}{2 a}\right)^{2}$ (with $a \neq 0$, $b \neq 0$ and $c \neq 0)$, we have that $0 \in f_{a, b, c}\left(\operatorname{Sing} f_{a, b, c}\right) \cap B\left(f_{a, b, c}\right)$.

(v) If $b^{2}-4 a c>0$ then we have the following two cases: $f_{a, b, c}=x y^{2}(a x y+b)$ and $f_{a, b, c}=$ $y\left(a x^{2} y^{2}+b x y+c\right)($ with $c \neq 0)$. In the case $f_{a, b, c}=x y^{2}(a x y+b)$, we have that $0 \in f_{a, b, c}\left(\operatorname{Sing} f_{a, b, c}\right)$. In the case $f_{a, b, c}=y\left(a x^{2} y^{2}+b x y+c\right)($ with $c \neq 0)$, we have that $f_{a, b, c}$ is a submersion and $0 \in S\left(f_{a, b, c}\right)$.

REMARK 3.3.2. Let $f_{a, b, c}: \mathbb{R}^{2} \rightarrow \mathbb{R}$ be a polynomial function as in Example 3.3.1. If we take $a=2, b=-9$ and $c=12$, then $b^{2}-4 a c<0$ and $b^{2}-3 a c>0$. Therefore, this polynomial is in the case (ii) of the Example 3.3.1 and, consequently, $f_{2,-9,12}$ is a submersion, $B\left(f_{2,-9,12}\right)=\emptyset$, but $0 \in S\left(f_{2,-9,12}\right)$. On these conditions on $a, b, c$, we obtain just the same polynomial considered in Tibăr and Zaharia [57, Example 3.4], i.e. the King-Tibăr-Zaharia's example. 


\section{Polynomial mappings}

\section{Contents}

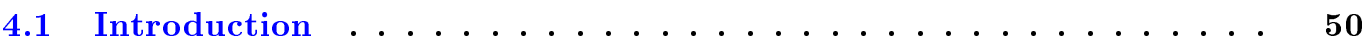

4.2 Integral closure of modules and $t$-regularity $\ldots \ldots \ldots \ldots$

4.2.1 Real integral closure of modules . . . . . . . . . . . . . . . 51

$4.2 .2 t$-regularity and polynomial mappings $\ldots \ldots \ldots \ldots \ldots \ldots$

4.3 Newton polyhedron and bifurcation values $\ldots \ldots \ldots \ldots \ldots$

4.3 .1 Newton polyhedron . . . . . . . . . . . . . . . 57

4.3.2 Newton polyhedron and polynomial mappings . . . . . . . . . . . 59

4.3.3 Non-degeneracy conditions at infinity . . . . . . . . . . . . . 62

\section{Brief Resume}

We consider two classes of polynomial mappings $f: \mathbb{R}^{n} \rightarrow \mathbb{R}^{p}$ : the class of fair polynomial mappings (Definition 4.2.4) and the class of Newton non-degenerate polynomial mappings (Definition 4.3.3).

For the class of fair polynomial mappings, we give an algebraic interpretation of the $t$ regularity condition (also Gaffney, Jelonek, Kuo-KOS and Rabier conditions) in terms of the theory of real integral closure of modules (Proposition 4.2.5, Theorem 4.2.6 and Remark 4.2.8).

We study the Newton non-degenerate polynomial mappings on a new non-degenerate condition. We give an approximation to the sets $\mathcal{N}_{\infty}(f)$ and $\mathcal{N} \mathcal{T}_{\infty}(f)$ in terms of a set that depends only on the Newton polyhedron of $f$ (Theorem 4.3.8 and Corollary 4.3.9). We also present some consequences of these last results and discuss some relations of this chapter with other works, in special with the works of Bivià-Ausina [2], Chen and Tibăr [8], Gaffney [18] and Némethi and Zaharia [40].

To finish, we give examples to illustrate the results of this chapter. 


\subsection{Introduction}

The main goal of this chapter is to study two classes of real polynomial mappings: the class of fair polynomial mappings (Definition 4.2.4) and the class of Newton non-degenerate polynomial mappings (Definition 4.3.3).

We remark that, since polynomial mappings are semi-algebraic mappings, the results of Chapters 2 and 3 hold for them.

The purpose here is to obtain for fair and Newton no-degenerate polynomial mappings new interpretations/informations about the bifurcation values and regularity conditions at infinity, which do not follow from the results of Chapters 2 and 3.

Since the treatment for each class is completely different than that of the other class, we structure this chapter as follows: in $\S 4.2$, we consider the class of fair polynomial mappings and, in $\S 4.3$, we consider the class of Newton non-degenerate polynomial mappings.

\subsection{Integral closure of modules and $t$-regularity}

We are interested in algebraic interpretations of $t$-regularity for the class of fair polynomial mappings. Our motivations for this study have been the works of Tibăr [53] and Gaffney [18]. Tibăr [53, Remark 2.9] observed that for complex polynomial functions $f: \mathbb{C}^{n} \rightarrow \mathbb{C}$, the $t$ regularity condition has an algebraic interpretation in terms of the theory of integral closure. For fair complex polynomial mappings, Gaffney [18, Proposition 5] described in terms of the theory of integral closure of modules a non-characteristic condition ([18, Definition 1]) which turns out to be equivalent to the $t$-regularity.

Following Gaffney, we define the "fair condition" for real polynomial mappings $f: \mathbb{R}^{n} \rightarrow$ $\mathbb{R}^{p}$ and we obtain another interpretation of $t$-regularity (consequently of Gaffney, Jelonek, Kuo-KOS and Rabier conditions) in terms of the theory of real integral closure of modules (Proposition 4.2.5 and Remark 4.2.8). Using this interpretation, we give in Theorem 4.2.6 a different proof of the equivalence between $t$-regularity and Gaffney condition ${ }^{1}$.

This section is organised as follows: in $§ 4.2 .1$, we define integral closure of modules (Definition 4.2.1) and discuss some equivalent definitions for Definition 4.2.1. Moreover, we present a result that describes the relative conormal in terms of the theory of integral closure of modules (Lemma 4.2.3). In $§ 4.2 .2$, we define fair polynomial mappings and prove the two main results of $\S 4.2$, Proposition 4.2.5 and Theorem 4.2.6. To finish, we present some examples and remarks in order to illustrate the results of this section. The results of this section have been published in [11].

\footnotetext{
${ }^{1}$ consequently, by the Theorem 2.4.8 and Corollary 2.4.9, Jelonek, Kuo-KOS and Rabier conditions.
} 


\subsubsection{Real integral closure of modules}

Let us denote by $\mathcal{A}_{n}$ the local ring of real analytic function germs at the origin in $\mathbb{R}^{n}$ and by $m_{n}$ its maximal ideal. We denote by $\mathcal{A}_{n}^{p}$ the free $\mathcal{A}_{n}$-module of rank $p$. We denote by $\mathcal{A}_{X, x}$ the local ring of real analytic function germs on the real analytic space germ $(X, x)$ and by $\mathcal{A}_{X, x}^{p}$ the free $\mathcal{A}_{X, x}$-module of rank $p$, i.e. $\mathcal{A}_{X, x}^{p}=\underbrace{\mathcal{A}_{X, x} \times \ldots \times \mathcal{A}_{X, x}}_{p \text { times }}$.

Given an analytic path $\phi:(\mathbb{R}, 0) \rightarrow(X, x)$, we set:

$$
\begin{aligned}
\phi^{*}: \mathcal{A}_{X, 0}^{p} & \longrightarrow \mathcal{A}_{1}^{p} \\
h & \longmapsto \phi^{*}(h)=h \circ \phi .
\end{aligned}
$$

In other words, from $\phi$ we have the following diagram:

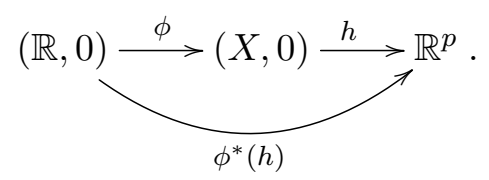

With the above notations, we have:

Definition 4.2.1 ([16, Definition 4.1]). Let $(X, x) \subset\left(\mathbb{R}^{n}, x\right)$ be a real analytic germ and let $M$ be an $A_{X, x}$-submodule of $\mathcal{A}_{X, x}^{p}$. The real integral closure of $M$, denoted by $\bar{M}$, is the set of elements $h \in \mathcal{A}_{X, x}^{p}$ such that for any analytic path $\phi:(\mathbb{R}, 0) \rightarrow(X, x)$, one has $\phi^{*}(h) \in \mathcal{A}_{1}\left(\phi^{*}(M)\right)$, where $\mathcal{A}_{1}\left(\phi^{*}(M)\right)$ denotes the $\mathcal{A}_{1}$-submodule of $\mathcal{A}_{1}^{p}$ generated by the elements $w \circ \phi, \forall w \in M$.

In the complex setting one has some equivalent definitions to the integral closure of modules, see Gaffney [16, Proposition 1.7, Proposition 1.11], which hold in the real setting with the exception of [16, Proposition 1.7], for which it is necessary to assume that the regular points of $X$ are dense in $X$, see Gaffney [16, page 318].

In the case that the regular points of $X$ are dense in $X$, an useful equivalent definition to the real integral closure of modules is that we may use in the Definition 4.2.1 only analytic paths on a dense Zariski-open subset of $X_{\text {reg }}^{2}$, see Gaffney, Trotman and Wilson [19, Proposition 1.4]. From this last equivalent definition, we have that the proof of the next criterion for the real integral closure of modules follows by the same arguments as in [17, Proposition 1.6]:

Lemma 4.2.2. Let $(X, x)$ be an equidimensional real analytic set. Suppose that the set of regular points of $X$ is dense in $X$. Let $N \subset M$ be $\mathcal{A}_{X, x}$-submodules of $\mathcal{A}_{X, x}^{p}$ and $h \in M$ such that $(h, N)=M$. Let $\mathcal{U}$ be a dense Zariski open subset of $X_{\mathrm{reg}}$. Then $h \in \bar{N}$ if and only if for any analytic paths $\phi:(\mathbb{R}, 0) \rightarrow(\mathcal{U}, x)$ and $\psi:(\mathbb{R}, 0) \rightarrow \mathbb{R}^{p} \cong \mathcal{L}\left(\mathbb{R}^{p}, \mathbb{R}\right)$ we have $\psi\left(\phi^{*}(h)\right) \in \mathcal{I}_{\psi}\left(\phi^{*}(N)\right)$, where $\mathcal{I}_{\psi}\left(\phi^{*}(N)\right)$ is the ideal in $\mathcal{A}_{1}$ generated by applying $\psi(s)$ to the generators of $\left(\phi^{*}(N)\right)$.

\footnotetext{
${ }^{2}$ Remember that we have denoted by $X_{\text {reg }}$ the regular points of $X$.
} 
There are interesting connections of integral closure theory with geometry, for instance Gaffney [16, 17], Teissier [52], Lejeune-Jalabert and Teissier [31], etc...

In special, the next result gives an interpretation of the relative conormal in terms of the integral closure of modules. It was formulated by Gaffney in [18, Proposition 4] in the complex setting but we can show that it holds in the real case. Under the notations of $\S 2.4 .2$, we have:

Lemma 4.2.3. Let $(X, x) \subset\left(\mathbb{R}^{m}, x\right)$ be the germ of an equidimensional real analytic set defined by $\tilde{F}:\left(\mathbb{R}^{m}, x\right) \rightarrow\left(\mathbb{R}^{p}, 0\right)$. Suppose that the regular points of $X$ are dense in $X$. Let $g:\left(\mathbb{R}^{m}, x\right) \rightarrow \mathbb{R}$ be a non-constant function and denote $G:=(\tilde{F}, g)$. Let $V \subset \mathbb{R}^{m}$ be a linear subspace. Then the following are equivalent:

(a) There exist $H \in C_{g, x}(X)^{3}$ such that $H \supset V$.

(b) $\overline{J M_{X}(G)_{V}} \varsubsetneqq \overline{J M_{X}(G)}$, where $J M_{X}(G)_{V}$ denotes the $\mathcal{A}_{X, x^{-}}$submodule of $\mathcal{A}_{X, x}^{p+1}$ generated by $\left\{\mathrm{D} G(x) v:=v_{1} \frac{\partial G}{\partial z_{1}}(x)+\ldots+v_{m} \frac{\partial G}{\partial z_{m}}(x) ; \forall v=\left(v_{1}, \ldots, v_{m}\right) \in V\right\}, J M_{X}(G)$ denotes the $\mathcal{A}_{X, x^{-}}$submodule of $\mathcal{A}_{X, x}^{p+1}$ generated by $\left\{\frac{\partial G}{\partial z_{1}}, \ldots, \frac{\partial G}{\partial z_{m}}\right\}$, and $\mathrm{D} G$ denotes the Jacobian of $G$.

Proof. We denote by $X_{0}$ the set of regular points of $X$ where $g_{\mid X}$ is a submersion.

"(a) $\Rightarrow(\mathrm{b})$ ". Suppose that there exists $H \in C_{g, x}(X)$ such that $V \subset H$. By Definition 2.4.3, this means that there exists a sequence $\left\{\left(x_{k}, H_{k}\right)\right\}_{k \in \mathbb{N}} \subset X_{0} \times \check{\mathbb{P}}^{m-1}$ with $T_{x_{k}}\left(g_{\mid X}^{-1}\left(g_{\mid X}\left(x_{k}\right)\right)\right) \subset H_{k}$, such that $\lim _{k \rightarrow \infty}\left(x_{k}, H_{k}\right)=(x, H)$. We may therefore take $\psi_{k}:=\left(\psi_{1 k}, \ldots, \psi_{(p+1) k}\right) \in \mathbb{R}^{p+1}$ such that $\psi_{k}\left(\mathrm{D} G\left(x_{k}\right)\right)=\left(a_{1 k}, \ldots, a_{m k}\right)$ and $H_{k}=\left\{x \in \mathbb{R}^{m} \mid \sum_{i=1}^{m} a_{i k} x_{i}=0\right\}$. Thus, by the Curve Selection Lemma [34, §3], there exist analytic curves $\phi:(\mathbb{R}, 0) \rightarrow\left(X_{0}, x\right)$, $\psi:(\mathbb{R}, 0) \rightarrow \mathbb{R}^{p+1}$ and an integer $l>0$ such that the vector

$$
\psi(s)(\mathrm{D} G(\phi(s)))=\left(a_{1}(s), \ldots, a_{m}(s)\right)
$$

defines a hyperplane $H_{\phi(s)}$ tangent to the fibre of $g_{\mid X}$ at $\phi(s)$, the number $l$ is the minimum of the orders of the first non-vanishing terms in $a_{1}(s), \ldots, a_{m}(s)$ and $\left(a_{1}, \ldots, a_{m}\right)=$ $\lim _{s \rightarrow 0} \frac{1}{s^{l}}(\psi(s) \mathrm{D} G(\phi(s)))$, where $H=\left\{x \in \mathbb{R}^{m} \mid \sum_{i=1}^{m} a_{i} x_{i}=0\right\}$.

Now, from the definition of $\mathcal{I}_{\psi} \phi^{*}\left(J M_{X}(G)_{H}\right)$, we have the equality $\mathcal{I}_{\psi} \phi^{*}\left(J M_{X}(G)_{H}\right)=$ $\mathcal{A}_{1}\{\psi(s)(\mathrm{D} G(\phi(s)) w) \mid w \in H\}$, and, since $\mathcal{I}_{\psi} \phi^{*}\left(J M_{X}(G)_{H}\right)$ is contained in $\mathcal{A}_{1}$, it follows that $\mathcal{I}_{\psi} \phi^{*}\left(J M_{X}(G)_{H}\right)$ is equal to $\mathcal{A}_{1}\left\{s^{q}\right\}$, for some $q \in \mathbb{N}$. From this last two equalities and by the facts that $\left(a_{1}, \ldots, a_{m}\right)=\lim _{s \rightarrow 0} \frac{1}{s^{l}}(\psi(s) \mathrm{D} G(\phi(s)))$ and that $H$ is defined by $\left(a_{1}, \ldots, a_{m}\right)$, we conclude that $\mathcal{I}_{\psi} \phi^{*}\left(J M_{X}(G)_{H}\right)=\mathcal{A}_{1}\left\{s^{q}\right\}$, for $q>l$.

On the other hand, again from $\left(a_{1}, \ldots, a_{m}\right)=\lim _{s \rightarrow 0} \frac{1}{s^{l}}(\psi(s) \mathrm{D} G(\phi(s)))$ and that $H$ is defined by $\left(a_{1}, \ldots, a_{m}\right)$, we have $\lim _{s \rightarrow 0} \frac{1}{s^{l}}(\psi(s)(\mathrm{D} G(\phi(s)) u)) \neq 0$, for any vector $u \in$ $\mathbb{R}^{m} \backslash H$. This last limit and the equality $\mathcal{I}_{\psi} \phi^{*}\left(J M_{X}(G)_{H}\right)=\mathcal{A}_{1}\left\{s^{q}\right\}$, for $q>l$, imply $\psi(s)(\mathrm{D} G(\phi(s)) u) \notin \mathcal{I}_{\psi} \phi^{*}\left(J M_{X}(G)_{H}\right)$. Therefore, from Lemma 4.2 .2 we conclude that

\footnotetext{
${ }^{3} C_{g, x}(X)$ as in $\S 2.4 .2$.
} 
$\mathrm{D} G(x) u \notin \overline{J M_{X}(G)_{H}}$, which implies $\overline{J M_{X}(G)_{H}} \varsubsetneqq \overline{J M_{X}(G)}$. In particular, since one has $J M_{X}(G)_{V} \subset J M_{X}(G)_{H}$, we conclude that $\overline{J M_{X}(G)_{V}} \varsubsetneqq \overline{J M_{X}(G)}$.

"(b) $\Rightarrow(\mathrm{a})$ ". Suppose that $\overline{J M_{X}(G)_{V}} \varsubsetneqq \overline{J M_{X}(G)}$. Then there is $\tilde{u} \in \mathbb{R}^{m} \backslash V$ such that $\mathrm{D} G(x) \tilde{u} \notin \overline{J M_{X}(G)_{V}}$. Thus, from Lemma 4.2 .2 , we may take $\psi:(\mathbb{R}, 0) \rightarrow \mathbb{R}^{p+1}$ and $\phi:(\mathbb{R}, 0) \rightarrow\left(X_{0}, x\right)$ such that $\psi(s)(\mathrm{D} G(\phi(s)) \tilde{u}) \notin \mathcal{I}_{\psi}\left(\phi^{*}\left(J M_{X}(G)_{V}\right)\right)$.

Since the ideals $\mathcal{I}_{\psi}\left(\phi^{*}\left(J M_{X}(G)\right)\right)$ and $\mathcal{I}_{\psi}\left(\phi^{*}\left(J M_{X}(G)_{V}\right)\right)$ are contained in $\mathcal{A}_{1}$, we may write $\mathcal{I}_{\psi}\left(\phi^{*}\left(J M_{X}(G)\right)\right)=\mathcal{A}_{1}\left\{s^{l}\right\}$ and $\mathcal{I}_{\psi}\left(\phi^{*}\left(J M_{X}(G)_{V}\right)\right)=\mathcal{A}_{1}\left\{s^{q}\right\}$, for integers $l, q \geq 0$. Moreover, we have $0 \leq l<q$ since $\psi(s)(\mathrm{D} G(\phi(s)) \tilde{u}) \notin \mathcal{I}_{\psi}\left(\phi^{*}\left(J M_{X}(G)_{V}\right)\right)$.

From the equality $\mathcal{I}_{\psi}\left(\phi^{*}\left(J M_{X}(G)\right)\right)=\mathcal{A}_{1}\left\{s^{l}\right\}$, the vector $a:=\lim _{s \rightarrow 0} \frac{1}{s^{l}}(\psi(s)(\mathrm{D} G(\phi(s))))$ is different from zero. If we take $H$ as the hyperplane defined by $a$, i.e. $H:=\left\{\left(x_{1}, \ldots, x_{m}\right) \mid\right.$ $\left.\sum_{j=1}^{m} a_{j} x_{j}=0\right\}$ then $H \in C_{g, x}(X)$ by construction and $H \supset V$ since $q>l$. These end our proof.

\subsection{2 $t$-regularity and polynomial mappings}

We begin with the definition of fair polynomial mapping. Then, we present some results for this class, which are a real counterpart of some results from [18].

\section{The "fair" condition}

As before (subsection Notation, page 27), we use coordinates $\left(x_{1}, \ldots, x_{n}\right)$ for the affine space $\mathbb{R}^{n}$, coordinates $\left[x_{0}: x_{1}: \ldots: x_{n}\right]$ for the projective space $\mathbb{P}^{n}$ and we shall consider the canonical embedding of $\mathbb{R}^{n}$ in $\mathbb{P}^{n}\left(\left(x_{1}, \ldots, x_{n}\right) \mapsto\left[1: x_{1}: \ldots: x_{n}\right]\right)$. We set $\mathbb{H}^{\infty}:=\left\{\left[x_{0}: \ldots\right.\right.$ : $\left.\left.x_{n}\right] \in \mathbb{P}^{n} \mid x_{0}=0\right\}$ the hyperplane at infinity.

Let $f=\left(f_{1}, \ldots, f_{p}\right): \mathbb{R}^{n} \rightarrow \mathbb{R}^{p}$ be a polynomial mapping and $\mathbb{X}:=\overline{\text { graph } f}$ the closure of the graph of $f$ in $\mathbb{P}^{n} \times \mathbb{R}^{p}$ (via the embedding of $\mathbb{R}^{n}$ in $\mathbb{P}^{n}$ ). We set $\mathbb{X}^{\infty}:=\mathbb{X} \cap\left(\mathbb{H}^{\infty} \times \mathbb{R}^{p}\right.$ ).

We denote by $\tilde{f}_{i}\left(x_{0}, x_{1}, \ldots, x_{n}\right)$ the homogenization of $f_{i}$ of degree $d_{i}:=\operatorname{deg} f_{i}$ in the variable $x_{0}$, for $1 \leqslant i \leqslant p$, and we set:

$$
\mathcal{Z}:=\bigcap_{i=1}^{p}\left\{\left(\left[x_{0}: x_{1}: \ldots: x_{n}\right],\left(t_{1}, \ldots, t_{p}\right)\right) \times \mathbb{P}^{n} \times \mathbb{R}^{p} \mid \tilde{F}_{i}\left(x_{0}, x_{1}, \ldots, x_{n}, t_{1}, \ldots, t_{p}\right)=0\right\}
$$

where $\tilde{F}_{i}\left(x_{0}, x_{1}, \ldots, x_{n}, t_{1}, \ldots, t_{p}\right):=\tilde{f}_{i}\left(x_{0}, x_{1}, \ldots, x_{n}\right)-t_{i} x_{0}^{d_{i}}=0$. From the equation (4.2), we set $\tilde{F}:=\left(\tilde{F}_{1}, \ldots, \tilde{F}_{p}\right)$.

Definition 4.2.4 (See Gaffney [18, page 158] in the complex case). We say that $f: \mathbb{R}^{n} \rightarrow \mathbb{R}^{p}$ is a fair polynomial if $\mathcal{Z}=\mathbb{X}$.

From the definitions of $\mathbb{X}$ and $\mathcal{Z}$, we have the inclusion $\mathbb{X} \subset \mathcal{Z}$ and the equality $\mathbb{X} \backslash\left(\mathbb{H}^{\infty} \times\right.$ $\left.\mathbb{R}^{p}\right)=\mathcal{Z} \backslash\left(\mathbb{H}^{\infty} \times \mathbb{R}^{p}\right)$. However, we do not have the equality $\mathbb{X}=\mathcal{Z}$ in general, see for instance Example 4.2.9. 
In the complex setting, Gaffney [18] works with the space $\mathcal{Z}$ assuming the equality $\mathcal{Z}=\mathbb{X}$, which he translates by " $f$ is fair" Gaffney [18, page 158]. This is imposed by the theory of integral closure of modules since "fair" implies that the regular points of $\mathcal{Z}$ are dense in $\mathcal{Z}$ and that $\mathcal{Z}$ is an equidimensional analytic set. See Example 4.2.9 in which $\mathbb{X} \neq \mathcal{Z}$ and therefore this technique does not apply, whereas Theorem 2.4.8 holds true.

\section{$t$-regularity and fair polynomial mappings}

Let $f=\left(f_{1}, \ldots, f_{p}\right): \mathbb{R}^{n} \rightarrow \mathbb{R}^{p}$ be a fair polynomial mapping. As in $\S 2.4 .2$ (page 29), we consider the charts $U_{j} \times \mathbb{R}^{p}$ of $\mathbb{P}^{n} \times \mathbb{R}^{p}$, where $U_{j}=\left\{x_{j} \neq 0\right\}, j=0,1, \ldots, n$, and we identify the chart $U_{0}$ with the affine space $\mathbb{R}^{n}$.

Let $z_{0} \in \mathbb{X}^{\infty}$. Up to some linear change of coordinates one may assume that $z_{0} \in \mathbb{X}^{\infty} \cap$ $\left(U_{n} \times \mathbb{R}^{p}\right)$. In the chart $U_{n} \times \mathbb{R}^{p}$, we take $y_{0}=x_{0} / x_{n}, \ldots, y_{n-1}=x_{n-1} / x_{n}$. In this coordinate system and since $f$ is fair, we have the following equality:

$$
\mathbb{X} \cap\left(U_{n} \times \mathbb{R}^{p}\right)=\bigcap_{i=1}^{p}\left\{\tilde{F}_{i}(y, t)=\tilde{f}_{i}\left(y_{0}, y_{1}, \ldots, y_{n-1}, 1\right)-t_{i} y_{0}^{d_{i}}=0\right\}
$$

where $\tilde{F}_{i}$ are as in the equation (4.2), and we have the following two results:

Proposition 4.2.5. Let $f: \mathbb{R}^{n} \rightarrow \mathbb{R}^{p}$ be a fair polynomial mapping. Then $f$ is $t$-regular at $z_{0} \in \mathbb{X}^{\infty}$ if and only if one of the following equivalent conditions is satisfied:

$$
\begin{gathered}
\partial \tilde{F} / \partial t_{i} \in \overline{\left\{\partial \tilde{F} / \partial y_{1}, \ldots, \partial \tilde{F} / \partial y_{n-1}\right\}}, \quad \forall i=1, \ldots, p . \\
\partial \tilde{F} / \partial t_{i} \in \overline{\left\{y_{0} \partial \tilde{F} / \partial y_{0}, \partial \tilde{F} / \partial y_{1}, \ldots, \partial \tilde{F} / \partial y_{n-1}\right\}}, \quad \forall i=1, \ldots, p .
\end{gathered}
$$

Proof. We show that $f$ is $t$-regular at $z_{0}$ iff the condition (4.3) is satisfied. Let $V:=\mathbb{R}^{n} \times 0 \subset$ $\mathbb{R}^{n} \times \mathbb{R}^{p}$ and let $g$ be the projection to the variable $y_{0}$. From Definitions 2.4.5 and 2.4.6, $z_{0}$ is a $t$-regular point if and only if there are no hyperplanes $H \supset V$ such that $H \in \mathcal{C}_{z_{0}}^{\infty}$. Applying Lemma 4.2 .3 to the mapping $G=(\tilde{F}, g)$ and to the set $V$, this is equivalent to the following condition:

$$
\frac{\partial G}{\partial t_{i}} \in \overline{\left\{\frac{\partial G}{\partial y_{0}}, \frac{\partial G}{\partial y_{1}}, \ldots, \frac{\partial G}{\partial y_{n-1}}\right\}} \text {, for } i=1, \ldots, p
$$

From the definition of integral closure of module (Definition 4.2.1), the equation (4.5) means that for any $\phi:(\mathbb{R}, 0) \rightarrow\left(\mathbb{X}, z_{0}\right)$, there exist $\lambda_{0}, \lambda_{1}, \ldots, \lambda_{n-1} \in \mathcal{A}_{1}$ such that:

$$
\left(\begin{array}{c}
\frac{\partial \tilde{F}}{\partial t_{i}}(\phi(s)) \\
0
\end{array}\right)=\lambda_{0}(s)\left(\begin{array}{c}
\frac{\partial \tilde{F}}{\partial y_{0}}(\phi(s)) \\
1
\end{array}\right)+\lambda_{1}(s)\left(\begin{array}{c}
\frac{\partial \tilde{F}}{\partial y_{1}}(\phi(s)) \\
0
\end{array}\right)+\cdots+\lambda_{n-1}(s)\left(\begin{array}{c}
\frac{\partial \tilde{F}}{\partial y_{n-1}}(\phi(s)) \\
0
\end{array}\right)
$$

which is in turn equivalent to (4.3). 
That (4.3) implies (4.4) is obvious. The converse is the same as Gaffney's proof of [18, Proposition 12] and Parusinski's proof of [42, Lemma 3.2].

By using the Proposition 4.2.5, we present for the class of fair polynomial mappings $f: \mathbb{R}^{n} \rightarrow \mathbb{R}^{p}$ the equivalence " $t$-regularity iff Gaffney (equivalently Jelonek, Kuo-KOS, Rabier) condition" with a proof in a different way than that of Theorem 2.4.8.

Theorem 4.2.6. A fair polynomial mapping $f: \mathbb{R}^{n} \rightarrow \mathbb{R}^{p}$ is t-regular at $z_{0} \in \mathbb{X}^{\infty}$ if and only if satisfies Gaffney condition at $z_{0}$.

Proof. Proposition 4.2.5 says that $t$-regularity at $z_{0}$ is equivalent to (4.4). In turn, by [16, Proposition 1.7] which holds over $\mathbb{R}$ too since $f$ is fair (see also Gaffney's remarks on the real setting [16, page 318$]$ ), the condition (4.4) is equivalent to:

$$
y_{0}^{d_{j}} \tilde{M}_{J, j}(\tilde{F}) \in \overline{\left\langle\tilde{M}_{I}(\tilde{F})\right\rangle}, j=1, \ldots, p,
$$

where $\left\langle\tilde{M}_{I}(\tilde{F})\right\rangle$ denotes the ideal generated by the maximal minors of the matrix whose columns are $\left(y_{0} \partial \tilde{F} / \partial y_{0}, \partial \tilde{F} / \partial y_{1}, \ldots, \partial \tilde{F} / \partial y_{n-1}\right)$, and $\tilde{M}_{J, j}(\tilde{F})$ is a minor of size $(p-1)$ of the same matrix with the $j$ th row deleted.

Using [16, Proposition 4.2], one has that (4.6) is equivalent to the existence of $C>0$ and a neighbourhood $U$ of $z_{0}$ such that:

$$
\sup _{J, j}\left\|y_{0}^{d_{j}}\right\|\left\|\tilde{M}_{J, j}(\tilde{F})(y, t)\right\| \leq C \sup _{I}\left\|\tilde{M}_{I}(\tilde{F})(y, t)\right\|, \text { for all }(y, t) \in U
$$

Dividing both sides of (4.7) by $\left\|y_{0}^{k}\right\|$, where $k=\sum_{l=1}^{p}\left(d_{l}-1\right)$, and from properties of the determinant function and the following relations between the partials of $f$ and the partials of $\tilde{F}$ :

$$
\begin{cases}\frac{\partial \tilde{F}_{j}}{\partial y_{i}} / y_{0}^{d_{j}-1}=\frac{\partial f_{j}}{\partial x_{i}}, & 1 \leq i \leq n-1,1 \leq j \leq p, \\ \frac{\partial \tilde{F}_{j}}{\partial t_{l}}=-y_{0}^{d_{j}} \delta_{l, j}, & 1 \leq j, l \leq p, \\ \frac{\partial \tilde{F}_{j}}{\partial y_{0}} / y_{0}^{d_{j}-1}=-\left(x_{1} \frac{\partial f_{j}}{\partial x_{1}}+\ldots+x_{n} \frac{\partial f_{j}}{\partial x_{n}}\right), & 1 \leq j \leq p,\end{cases}
$$

we obtain

$$
\sup _{J, j}\left\|1 / x_{n}\right\|\left\|M_{J, j}^{\prime}(f)(x)\right\| \leq C \sup _{I}\left\|M_{I}^{\prime}(f)(x)\right\|,
$$

where $M_{J, j}^{\prime}(f)$ and $M_{I}^{\prime}(f)(x)$ are defined as follows: if $I=\left(i_{1}<\ldots<i_{p}\right)$ with $i_{1} \neq 1$, i.e., if the minor $\tilde{M}_{I}(\tilde{F})$ indexed by $I$ does not contain the column $y_{0} \partial \tilde{F} / \partial y_{0}$ then $M_{I}^{\prime}(f)$ is just the maximal minor indexed by $I^{\prime}=\left(i_{1}-1<\ldots<i_{p}-1\right)$ of the Jacobian matrix $\mathrm{D} f$ (i.e. $\left.M_{I}^{\prime}(f)=M_{I^{\prime}}(\mathrm{D} f)\right)^{4}$, and otherwise, $M_{I}^{\prime}(f)(x)$ is defined as the minor maximal of the matrix

\footnotetext{
${ }^{4}$ see page 12 to the definition of maximal minor.
} 
$\left(\partial f / \partial x_{1}, \ldots, \partial f / \partial x_{n-1}, \sum_{l=1}^{n} \frac{x_{l}}{x_{n}} \partial f / \partial x_{l}\right)$ indexed by $I^{\prime}=\left(i_{2}-1<i_{3}-1<\ldots<i_{p-1}-1<\right.$ $n)$. A similar substitution should be made to define the $M_{J, j}^{\prime}(f)$ terms.

Using the fact that $\left\|x_{n}\right\| \geq\left\|x_{j}\right\|$, for $j=1, \ldots, n$, the inequality (4.8) is equivalent to the Gaffney condition (Definition 2.3.2).

Remark 4.2.7. We observe that Proposition 4.2.5 and Theorem 4.2.6 have been obtained by Gaffney [18] in the setting of fair complex polynomial mappings $f: \mathbb{C}^{n} \rightarrow \mathbb{C}^{p}$.

REMARK 4.2.8. From Theorem 2.4.8 and Corollary 2.4.9, we may change the $t$-regularity condition in Proposition 4.2.5 with the Gaffney, or Jelonek, or Kuo-KOS, or Rabier conditions (Definition 2.4.1).

\section{Examples}

We start with an example of a mapping that is not fair:

EXAmple 4.2.9. Let $f: \mathbb{R}^{3} \rightarrow \mathbb{R}^{2}, f\left(x_{1}, x_{2}, x_{3}\right)=\left(x_{1}^{2}, x_{1} x_{2}\right)$. We have $\mathbb{X}^{\infty}=X_{1} \cup X_{2}$, where $X_{1}=\left\{\left(\left[0: 0: x_{2}: x_{3}\right],\left(0, t_{2}\right)\right) \in \mathbb{P}^{3} \times \mathbb{R}^{2}\right\}$ and $X_{2}=\left\{\left([0: 0: 0: 1],\left(t_{1}, t_{2}\right)\right) \in \mathbb{P}^{3} \times \mathbb{R}^{2} \mid t_{1}>0\right\}$. On the other hand, we have $\mathcal{Z}^{\infty}:=\mathcal{Z} \cap\left(\mathbb{H}^{\infty} \times \mathbb{R}^{2}\right)=\left\{\left(\left[0: 0: x_{2}: x_{3}\right],\left(t_{1}, t_{2}\right)\right) \in \mathbb{P}^{3} \times \mathbb{R}^{2}\right\}$. Thus $\mathbb{X}^{\infty} \varsubsetneqq \mathcal{Z}^{\infty}$ and consequently $\mathbb{X} \varsubsetneqq \mathcal{Z}$. By straightforward computations, one gets that $f$ is not "fair" at any point $z_{0} \in \mathbb{X}^{\infty}$. Therefore we cannot use the approach of this chapter for $f$. Nevertheless, we still have the equivalence of $t$-regularity with Gaffney condition (Theorem 2.4.8), the structure and fibration theorems (Theorem 3.1.1, items (a) and (b)). Moreover, by straightforward computations, we obtain $f(\operatorname{Sing} f)=\{(0,0)\}$ and $B(f)=\mathcal{N}(f)=S(f)=$ $\tau\left(X_{1}\right)=\left\{\left(0, t_{2}\right) ; t_{2} \in \mathbb{R}\right\}$.

We use the above example to explain the following geometric fact about the sets $\operatorname{Im} f$ and $\overline{\operatorname{Im} f}$ :

EXAMPLE 4.2.10. Let $f: \mathbb{R}^{3} \rightarrow \mathbb{R}^{2}$ as in the last example, i.e, $f\left(x_{1}, x_{2}, x_{3}\right)=\left(x_{1}^{2}, x_{1} x_{2}\right)$. We have $f(\operatorname{Sing} f)=\{(0,0)\}$ and image of $f$, denoted here by $\operatorname{Im} f$, is the set $\operatorname{Im} f=\left\{\left(t_{1}, t_{2}\right) \in \mathbb{R}^{2} \mid\right.$ $\left.t_{1}>0\right\} \cup\{(0,0)\}$. Therefore, for any point $\left(t_{1}, 0\right) \in \mathbb{R}^{2}$ with $t_{1} \neq 0$, we have $\left(t_{1}, 0\right) \in \overline{\operatorname{Im} f} \backslash \operatorname{Im} f$, which implies $\left(t_{1}, 0\right) \in B(f)$ (this last affirmation follows directly from the definition of $B(f)$ ). Consequently, since $\left(t_{1}, 0\right) \notin f\left(\operatorname{Sing} f\right.$ ) (for any $t_{1} \neq 0$ ), we have the these points are only approximated by $S(f)$ or $\mathcal{N}_{\infty}(f)$.

Notice that the inclusions $\overline{\operatorname{Im} f} \backslash \operatorname{Im} f \subset S(f)$ and $\overline{\operatorname{Im} f} \backslash \operatorname{Im} f \subset \mathcal{N}_{\infty}(f)$ occur for any $C^{2}$ mapping $f: \mathbb{K}^{n} \rightarrow \mathbb{K}^{p}$ (in fact, these inclusions follow respectively by Theorem 3.1.1 item (a) and Theorem 2.3.5). However, only by the definitions of $S(f)$ and $\mathcal{N}_{\infty}(f)$, it is not easy to see that $\overline{\operatorname{Im} f} \backslash \operatorname{Im} f \subset S(f)$ and $\overline{\operatorname{Im} f} \backslash \operatorname{Im} f \subset \mathcal{N}_{\infty}(f)$ for $C^{2}$ mappings $f: \mathbb{K}^{n} \rightarrow \mathbb{K}^{p}$.

\subsection{Newton polyhedron and bifurcation values}

We introduce a Newton non-degeneracy condition at infinity for polynomial mappings $F: \mathbb{R}^{n} \rightarrow \mathbb{R}^{k}$ (Definition 4.3.3). Chen [6] introduced in his thesis the notion of non-degeneracy 
for mixed polynomial mappings. We have worked out together a part of the settings and results (Chen, Dias and Tibăr [7]).

The non-degeneracy for polynomial mappings (Definition 4.3.3) is designed to study the bifurcation values of $F$ and specially the case of non-convenient polynomial mappings (Definition 4.3.1). Thus, under this non-degeneracy condition, we obtain a result on the bifurcation values of $F$ in terms of the critical values of the restrictions $F_{\triangle}$ to certain faces of the Newton polyhedron of the components of $F$ (Theorem 4.3.8). This extends to mappings results obtained before for mixed functions by Chen and Tibăr in [8, Theorem 1.1 (a)], and previously by Némethi and Zaharia in [40, Theorem 2] for complex polynomial functions $f: \mathbb{C}^{n} \rightarrow \mathbb{C}$.

We structured this section as follows: in $§ 4.3 .1$, we define the Newton polyhedron and the Newton non-degeneracy for polynomial mappings $F: \mathbb{R}^{n} \rightarrow \mathbb{R}^{k}$. The main results for Newton non-degenerate mappings will be presented in $\S 4.3 .2$. In $\S 4.3 .3$, we compare the Newton non-degeneracy presented here (Definition 4.3.3) with the Newton non-degeneracy used by Bivià-Ausina [2] and we also compare the Definition 4.3.3 with the classical definition of Khovanskiı [26]. Some examples are presented in order to illustrate the results.

\subsubsection{Newton polyhedron}

The use of the Newton polyhedron to study the bifurcation values appears in Broughton $[4,5]$, Némethi and Zaharia [40], Pham [47] in the case of complex polynomial functions and in Chen and Tibăr [8] for mixed polynomial functions (see also Chen [6]). Here, we present the Newton non-degeneracy in the setting of polynomial mappings $f: \mathbb{R}^{n} \rightarrow \mathbb{R}^{p}$.

Let $f: \mathbb{R}^{n} \rightarrow \mathbb{R}$ be a non-constant polynomial function. We write $f(x)=\sum_{\nu} c_{\nu} x^{\nu}$, where $\nu=\left(\nu_{1}, \cdots, \nu_{n}\right) \in \mathbb{N}^{n}$ and $x^{\nu}=x_{1}^{\nu_{1}} \cdots x_{n}^{\nu_{n}}$.

Definition 4.3.1. Let $f: \mathbb{R}^{n} \rightarrow \mathbb{R}$ be a non-constant polynomial function. The support of $f$ is defined as $\operatorname{supp}(f):=\left\{\nu \in \mathbb{N}^{n} \mid c_{\nu} \neq 0\right\}$. We say that $f$ is convenient if the intersection of $\operatorname{supp}(f)$ with each coordinate axis is non-empty. We denote by $\overline{\operatorname{supp}(f)}$ the convex hull of the set $\operatorname{supp}(f) \backslash\{0\}$. The Newton polyhedron of $f$, denoted by $\Gamma_{0}(f)$, is the convex hull of the set $\{0\} \cup \operatorname{supp}(f)$. The Newton boundary at infinity of $f$, denoted by $\Gamma^{+}(f)$, is the union of the faces of the polyhedron $\Gamma_{0}(f)$ which do not contain the origin. By "face" we mean face of any dimension.

Let $\triangle$ be a face of $\overline{\operatorname{supp}(f)}$. The restriction of $f$ to $\triangle \cap \operatorname{supp}(f)$, denoted by $f_{\triangle}$, is defined as follows $f_{\triangle}(x):=\sum_{\nu \in \triangle} \operatorname{supp}(f) c_{\nu} x^{\nu}$.

Let us recall from Némethi and Zaharia [40] the definition of "bad" faces of $f$, which we call here "atypical".

Definition 4.3.2. Let $f: \mathbb{R}^{n} \rightarrow \mathbb{R}$ be a non-constant polynomial function. A face $\triangle$ of $\overline{\operatorname{supp}(f)}$ is called atypical if the following condition is verified:

(a) There exists a hyperplane $H \subset \mathbb{R}^{n}$ defined by $a_{1} x_{1}+\cdots+a_{n} x_{n}=0$ (where $x_{1}, \ldots, x_{n}$ are the coordinates in $\mathbb{R}^{n}$ ) such that: 
(i) there exist $1 \leq i, j \leq n$ with $a_{i}<0$ and $a_{j}>0$,

(ii) $H \cap \overline{\operatorname{supp}(f)}=\triangle$.

The face of $\triangle \subseteq \overline{\operatorname{supp}(f)}$ is called strictly atypical if moreover we have

(b) The affine subspace of the same dimension spanned by $\triangle$ contains the origin.

Let us now consider a polynomial mapping $F=\left(f_{1}, \ldots, f_{k}\right): \mathbb{R}^{n} \rightarrow \mathbb{R}^{k}, n \geq k$. For a vector $\mathbf{p}=\left(p_{1}, \ldots, p_{n}\right) \in \mathbb{Z}^{n}$, we set $p:=\min _{1 \leq i \leq n} p_{i}$, and we suppose $p<0$. Let $l_{\mathbf{p}}: \mathbb{R}^{n} \rightarrow \mathbb{R}$ be the function $l_{\mathbf{p}}(v)=\sum_{i=1}^{n} p_{i} v_{i}$, where $v \in \mathbb{R}^{n}$, and we consider the restriction of $l_{\mathbf{p}}$ on $\overline{\operatorname{supp}\left(f_{i}\right)}$.

Let us denote by $\triangle_{\mathbf{p}}^{j}$ the unique maximal face ${ }^{5}$ of $\overline{\operatorname{supp}\left(f_{j}\right)}$ where $l_{\mathbf{p}}$ takes its minimal value $d_{\mathbf{p}}^{j}$, for $1 \leq j \leq k$. For some multi-index $I \subset\{1, \ldots, k\}$, we set $F_{\triangle_{\mathbf{p}}}=\left(f_{\triangle_{\mathbf{p}}^{j}}\right)_{j \in I}$, where $f_{\triangle_{\mathbf{p}}^{j}}$ denotes the restriction of $f_{j}$ to the face $\triangle_{\mathbf{p}}^{j}$. We define:

$$
\mathrm{N}_{\mathbf{p}}:=\left\{j \in\{1, \ldots, k\} \mid \triangle_{\mathbf{p}}^{j} \text { is a face of } \Gamma^{+}\left(f_{j}\right) \text { and } d_{\mathbf{p}}^{j}<0\right\}
$$

Definition 4.3.3. We say that a polynomial mapping $F: \mathbb{R}^{n} \rightarrow \mathbb{R}^{k}$ is Newton non-degenerate at infinity, resp. Newton strongly non-degenerate at infinity, if for any vector $\mathbf{p} \in \mathbb{Z}^{n} \backslash\{0\}$ with $p<0$ and such that $\mathrm{N}_{\mathbf{p}} \neq \emptyset$, the following condition is satisfied:

$$
\operatorname{Sing} F_{\triangle_{\mathbf{p}}} \cap\left\{x \in \mathbb{R}^{n} \mid f_{\triangle_{\mathbf{p}}^{j}}(x)=0, \forall j \in \mathrm{N}_{\mathbf{p}}\right\} \cap\left(\mathbb{R}^{*}\right)^{n}=\emptyset
$$

respectively

$(* *) \quad \operatorname{Sing} F_{\triangle_{\mathbf{p}}} \cap\left(\mathbb{R}^{*}\right)^{n}=\emptyset$,

where $\mathbb{R}^{*}=\mathbb{R}-\{0\}$.

In the following we shall abbreviately write "non-degenerate" or "strongly non-degenerate". REMARK 4.3.4. Let $F=\left(f_{1}, \ldots, f_{k}\right): \mathbb{R}^{n} \rightarrow \mathbb{R}^{k}$ be a polynomial function. Let $q_{i}$ be the number of monomials of $f_{i}, i=1, \ldots, k$. Then the cardinality of the set $\left\{F_{\triangle_{\mathbf{p}}} \mid \mathbf{p} \in \mathbb{Z}^{n} \backslash 0\right\}$ is at most $\left(2^{q_{1}}-1\right)\left(2^{q_{2}}-1\right) \ldots\left(2^{q_{k}}-1\right)$. In particular, these says that the Newton non-degeneracy condition of $F$ is given by a finite number of conditions.

Definition 4.3.5. Let $F=\left(f_{1}, \ldots, f_{k}\right): \mathbb{R}^{n} \rightarrow \mathbb{R}^{k}$ be a polynomial mapping and let $\mathbf{p} \in$ $\mathbb{Z}^{n} \backslash 0$. If $\triangle_{\mathbf{p}}^{i}$ is an atypical (respectively strictly atypical) face of $f_{i}$ for all $i$, we say that $\triangle_{\mathbf{p}}=\triangle_{\mathbf{p}}^{1} \times \triangle_{\mathbf{p}}^{2} \times \cdots \times \triangle_{\mathbf{p}}^{k}$ is an atypical (respectively strictly atypical) face of $F$. We denote by $\mathscr{A}(F)$ the set of atypical faces of $F$.

REMARK 4.3.6. If $F$ is non-degenerate (respectively strongly non-degenerate) and $\triangle$ is an atypical face of $F$, then the mapping $F_{\triangle}$ is also non-degenerate at infinity (respectively strongly non-degenerate at infinity).

\footnotetext{
${ }^{5}$ "maximal face" means with respect to the inclusion of faces.
} 
Definition 4.3.7. We say that $F$ depends effectively on all the variables, if for every variable $z_{i}$ there exists some $j \in\{1, \ldots, k\}$ such that $f_{j}$ depends effectively on $z_{i}$.

\subsubsection{Newton polyhedron and polynomial mappings}

In the proof of the next theorem, we will use the following notations: consider $I \subset$ $\{1, \ldots, n\}$ a multi-index, we set the following three notations:

$(\mathrm{N}-1) \mathbb{R}^{I}:=\left\{\left(x_{1}, \ldots, x_{n}\right) \in \mathbb{R}^{n} \mid x_{i}=0, i \notin I\right\}$.

$(\mathrm{N}-2) F^{I}:=F_{\mid \mathbb{R}^{I}}$ the restriction of $F$ on $\mathbb{R}^{I}$.

$(\mathrm{N}-3)\left(\mathbb{R}^{*}\right)^{I}:=\left\{\left(x_{1}, \ldots, x_{n}\right) \in \mathbb{R}^{n} \mid x_{i}=0\right.$ iff $\left.i \notin I\right\}$.

Theorem 4.3.8. Let $F: \mathbb{R}^{n} \rightarrow \mathbb{R}^{k}, n \geq k$, such that $F(0)=0$. If $F$ is Newton non-degenerate at infinity and depends effectively on all the variables then:

$$
\mathcal{N}_{\infty}(F) \subset\left(\mathbb{R}^{k} \backslash\left(\mathbb{R}^{*}\right)^{k} \cup \underset{\triangle \in \mathscr{A}(f)}{\cup} F_{\triangle}\left(\operatorname{Sing} F_{\triangle} \cap\left(\mathbb{R}^{*}\right)^{n}\right)\right),
$$

where $\mathscr{A}(F)$ is the set of "atypical faces" of $F$ (Definition 4.3.5), and $\mathcal{N}_{\infty}(f)$ is defined as in Definition 2.3.1.

Proof. We will show that $\mathcal{N}_{\infty}(F) \cap\left(\mathbb{R}^{*}\right)^{k} \subset \underset{\triangle \in \mathscr{A}(F)}{\cup} F_{\triangle}\left(\operatorname{Sing} F_{\triangle} \cap\left(\mathbb{R}^{*}\right)^{n}\right)$, which clearly implies the expression (4.10).

Let $t=\left(t_{1}, \ldots, t_{k}\right) \in \mathcal{N}_{\infty}(F) \cap\left(\mathbb{R}^{*}\right)^{k}$. By definition of $\mathcal{N}_{\infty}(F)^{6}$, there exist analytic paths $\phi:] 0, \varepsilon\left[\rightarrow \mathbb{R}^{n}, \varphi=\left(\varphi_{1}, \ldots, \varphi_{k}\right):\right] 0, \varepsilon\left[\rightarrow \mathbb{R}^{k}\right.$, with the properties $\|\varphi(s)\|=1$, $\lim _{s \rightarrow 0}\|\phi(s)\|=\infty$ and $\lim _{s \rightarrow 0} f(\phi(s))=t$, so that one has:

$$
\lim _{s \rightarrow 0}\left\|\phi_{i}(s)\right\|\left\|\varphi_{1}(s) \frac{\partial f_{1}}{\partial x_{i}}(\phi(s))+\ldots+\varphi_{k}(s) \frac{\partial f_{k}}{\partial x_{i}}(\phi(s))\right\|=0 .
$$

We consider the following set $L:=\left\{j \in\{1, \ldots, n\} \mid \phi_{j}(s) \not \equiv 0\right\}$. Since $\lim _{s \rightarrow 0}\|\phi(s)\|=\infty$, one has $L \neq \emptyset$ and, for each $j \in L$, we write:

$$
\phi_{j}(s)=x_{j} s^{p_{j}}+\text { h.o.t., with } x_{j} \in \mathbb{R}, x_{j} \neq 0 \text { and } p_{j} \in \mathbb{Z} \text {. }
$$

We may assume (eventually after a change of coordinates) that $L=\{1, \ldots, m\}$ and $p=p_{1} \leq p_{2} \leq \cdots \leq p_{m}$ (note that since $\lim _{s \rightarrow 0}\|\phi(s)\|=\infty$, one has $p<0$ ).

From the numbers that appear in the equation (4.12), i.e. from $x_{1}, \ldots, x_{m}$ and $p_{1}, \ldots, p_{m}$, we set: $\mathrm{x}_{0}:=\left(x_{1}, \ldots, x_{m}, 0, \ldots, 0\right) \in\left(\mathbb{R}^{*}\right)^{L}$ and $\mathbf{p}:=\left(p_{1}, \ldots, p_{m}, g, \ldots, g\right) \in \mathbb{Z}^{n}$ with $g \in \mathbb{N}$ big enough. As in page 57 , we consider the linear function $l_{\mathbf{p}}$, where $\mathbf{p}$ is just the last vector defined in this paragraph.

\footnotetext{
${ }^{6}$ we are using here the Rabier function to generate the set $\mathcal{N}_{\infty}(F)$.
} 
Let $\triangle_{\mathbf{p}}^{i L}$ be the maximal face of $\overline{\operatorname{supp}\left(f_{i}^{L}\right)}$ where $l_{\mathbf{p}}$ restricted to $\overline{\operatorname{supp}\left(f_{i}^{L}\right)}$ takes its minimal value, which we denote by $d_{\mathbf{p}}^{i L}$. We observe that, by definition of the vector $\mathbf{p}$ and by definition of $f_{i}^{L}$, one has $\triangle_{\mathbf{p}}^{i L}=\triangle_{\mathbf{p}}^{i}, d_{\mathbf{p}}^{i L}=d_{\mathbf{p}}^{i}$, and consequently $f_{i \triangle_{\mathbf{p}}^{i L}}^{L}=f_{i \triangle_{\mathbf{p}}^{i}}{ }^{7}$ (in fact, for any $\left(v_{1}, \ldots, v_{n}\right) \in \overline{\operatorname{supp}\left(f_{i}\right)} \backslash \overline{\operatorname{supp}\left(f_{i}^{L}\right)}$, the value of $\sum_{i=1}^{m} p_{i} v_{i}+g \sum_{i=m+1}^{n} v_{i}$ is greater than $d_{\mathbf{p}}^{i L}$, $\forall i=1, \ldots, k)$. Thus, we will denote $\triangle_{\mathbf{p}}^{i L}\left(\operatorname{resp} . d_{\mathbf{p}}^{i L}\right)$ only by $\triangle_{\mathbf{p}}^{i}\left(\right.$ resp. $\left.d_{\mathbf{p}}^{i}\right)$.

Putting everything of the last two paragraph together, we may write:

$$
f_{i}(\phi(s))=f_{i}^{L}(\phi(s))=f_{i \triangle \mathbf{p}}^{L}\left(\mathrm{x}_{0}\right) s^{d_{\mathbf{p}}^{i}}+\text { h.o.t., } i=1, \ldots, k .
$$

Note that, since $\lim _{s \rightarrow 0} f_{i}(\phi(s))=t_{i}$ and $t_{i} \neq 0$, one has $d_{\mathbf{p}}^{i} \leq 0$ in the equation (4.13).

If $\varphi_{i}(s) \not \equiv 0$, one writes $\varphi_{i}(s)=\varphi_{i} s^{q_{i}}+$ h.o.t., $\varphi_{i} \in \mathbb{R}, \varphi_{i} \neq 0$ and $q_{i} \geq 0$. Let $I:=\{i \in$ $\left.\{1, \ldots, k\} \mid q_{i}+d_{\mathbf{p}}^{i}=\min _{1 \leq j \leq k}\left\{q_{j}+d_{\mathbf{p}}^{j}\right\}\right\}$. From the equation (4.11), for any $l=1, \ldots, n$, one has:

$$
\sum_{i \in I} \varphi_{i} x_{l} \frac{\partial f_{i \triangle \triangle_{\mathbf{p}}^{i}}^{L}}{\partial x_{l}}\left(\mathrm{x}_{0}\right) s^{q_{i}+d_{\mathbf{p}}^{i}}+\text { h.o.t. } \rightarrow 0 .
$$

Since $\|\varphi(s)\|=1$, we have $\min _{1 \leq i \leq n} q_{i}=0$ and therefore $q_{i}+d_{\mathbf{p}}^{i} \leq 0$, for every $i \in I$. So, the expression (4.14) gives:

$$
\sum_{i \in I} \varphi_{i} x_{l} \frac{\partial f_{i \triangle \triangle_{\mathbf{p}}^{i}}^{L}}{\partial x_{l}}\left(\mathrm{x}_{0}\right)=0 .
$$

Let $\mathrm{x}_{1}:=\left(x_{1}, \ldots, x_{m}, 1, \ldots, 1\right)$. From equation (4.15), from the definitions of the vectors $\mathrm{x}_{0}$ and $\mathrm{x}_{1}$, and by the equality $f_{i \triangle \mathbf{p}}^{L}=f_{i \triangle \mathbf{p}}^{i}$, we conclude that $\mathrm{x}_{1} \in \operatorname{Sing} F_{\triangle_{\mathbf{p}}} \cap\left(\mathbb{R}^{*}\right)^{n}$. The last equality (i.e., the equality $f_{i \triangle \triangle_{\mathbf{p}}^{i}}^{L}=f_{i \triangle \mathbf{p}}$ ) is explained in the paragraph before the equation (4.13).

Suppose $\mathrm{N}_{\triangle_{\mathbf{p}}}:=\left\{j \in\{1, \ldots, k\} \mid \triangle_{\mathbf{p}}^{j}\right.$ is a face of $\Gamma^{+}\left(f_{j}\right)$ and $\left.d_{\mathbf{p}}^{j}<0\right\} \neq \emptyset$. From the equation (4.13), by definitions of the vectors $\mathrm{x}_{0}$ and $\mathrm{x}_{1}$, and by equality $f_{i \triangle_{\mathbf{p}}^{i}}^{L}=f_{i \triangle_{\mathbf{p}}^{i}}$, we have $f_{j \triangle_{\mathbf{p}}^{j}}\left(\mathrm{x}_{1}\right)=0$, for all $j \in \mathrm{N}_{\triangle_{\mathbf{p}}}$. This implies that $\mathrm{x}_{1} \in \operatorname{Sing} F_{\triangle_{\mathbf{p}}} \cap\left\{x \in \mathbb{R}^{n} \mid f_{j \triangle_{\mathbf{p}}^{j}}(x)=0, \forall j \in\right.$ $\left.\mathrm{N}_{\triangle_{\mathbf{p}}}\right\} \cap\left(\mathbb{R}^{*}\right)^{n}$, which contradicts the Newton non-degeneracy of $F$.

Else, we conclude that $\mathrm{N}_{\triangle_{\mathbf{p}}}=\emptyset$. In the first paragraph after the equation (4.13), we have seen that $d_{i}^{\mathbf{p}} \leq 0$, for $i=1, \ldots, k$. However, sine $\mathrm{N}_{\triangle_{\mathbf{p}}}=\emptyset$ we have $d_{i}^{\mathbf{p}}=0, \forall i$ (in fact, if $d_{i}^{\mathbf{p}}<0$ for some $i$, this implies that $\triangle_{\mathbf{p}}^{j}$ is a face of $\Gamma^{+}\left(f_{j}\right)$, which contradicts $\mathrm{N}_{\triangle_{\mathbf{p}}}=\emptyset$, see also [8, Lemma 3.1, item (a)]).

Consider the hyperplane $H:=\left\{x=\left(x_{1}, \ldots, x_{n}\right) \in \mathbb{R}^{n} \mid \sum_{i=1}^{m} p_{i} x_{i}+g \sum_{i=m+1}^{n} x_{i}=0\right\}$, where the numbers $p_{1}, \ldots, p_{m}$ and $g$ are the same numbers which appear in the definition of p. We have obtained that $d_{\mathbf{p}}^{i}=0$, for $i=1, \ldots, k$. This implies that $\triangle_{\mathbf{p}}^{i}=\overline{\operatorname{supp}\left(f_{i}\right)} \cap H$, for $i=1, \ldots, k$, which shows us that the condition (a)(ii) of Definition 4.3.2 is satisfied for all $i$.

Let us suppose that there exists $j$ such that $\triangle_{\mathbf{p}}^{j}$ does not satisfy condition (a)-(i) of

\footnotetext{
${ }^{7}$ the definitions of $\triangle_{\mathbf{p}}^{i}, d_{\mathbf{p}}^{i}$ and $f_{i \triangle}{ }_{\mathbf{p}}^{i}$ is on the page 57 .
} 
Definition 4.3.2. Then, from definition of the set $L$, one has $m=n$ and $p_{i} \leq 0$, for all $1 \leq i \leq n$. By hypothesis, $F$ depends effectively on the variable $x_{1}$, which implies that there exists some $j \in\{1, \ldots, k\}$ such that $f_{j}$ depends effectively on the variable $x_{1}$. Since $p_{1}<0$, one obtains that $d_{\mathbf{p}}^{j}<0$. But this contradicts $d_{\mathbf{p}}^{j}=0$.

Therefore, one has that $\triangle_{\mathbf{p}}$ is an atypical face of $F$. Moreover, since $d_{\mathbf{p}}^{i}=0$, for $i=$ $1, \ldots, k$, we obtain $t=F_{\triangle_{\mathbf{p}}}\left(\mathrm{x}_{1}\right) \in F_{\triangle_{\mathbf{p}}}\left(\operatorname{Sing} F_{\triangle_{\mathbf{p}}} \cap\left(\mathbb{R}^{*}\right)^{n}\right)$, which finishes our proof. The last equality follows from the equality (4.13), from the definitions of $\mathrm{x}_{0}$ and $\mathrm{x}_{1}$, and by equality $f_{i \triangle \mathbf{p}}^{L}=f_{i \triangle \triangle_{\mathbf{p}}^{i}}$

As a direct consequence of Theorem 4.3.8 and Corollary 2.4.9, we have

Corollary 4.3.9. Let $F: \mathbb{R}^{n} \rightarrow \mathbb{R}^{k}, n \geq k$, such that $F(0)=0$. If $F$ is Newton nondegenerate at infinity and depends effectively on all the variables then:

$$
\mathcal{N} \mathcal{T}_{\infty}(F) \subset\left(\mathbb{R}^{k} \backslash\left(\mathbb{R}^{*}\right)^{k} \cup \underset{\triangle \in \mathscr{A}(f)}{\cup} F_{\triangle}\left(\operatorname{Sing} F_{\triangle} \cap\left(\mathbb{R}^{*}\right)^{n}\right)\right) .
$$

where $\mathcal{N} \mathcal{T}_{\infty}(f)$ is defined as in Corollary 2.4.9.

Now, let us give here two consequences of the Theorem 4.3.8.

Proposition 4.3.10. If $F: \mathbb{R}^{n} \rightarrow \mathbb{R}^{k}$ is strongly non-degenerate at infinity, then $F(\operatorname{Sing} F) \cap$ $\left(\mathbb{R}^{*}\right)^{k}$ and $\mathcal{N}_{\infty}(F) \cap\left(\mathbb{R}^{*}\right)^{k}$ are bounded.

Proof. Firstly, we will prove by contradiction that $F(\operatorname{Sing} F) \cap\left(\mathbb{R}^{*}\right)^{k}$ is bounded. Thus, suppose that $F(\operatorname{Sing} F) \cap\left(\mathbb{R}^{*}\right)^{k}$ is not bounded. From the Curve Selection Lemma (Milnor $[34, \S 3])$, there exist analytic paths $\phi:] 0, \epsilon\left[\rightarrow \operatorname{Sing} F\right.$ and $\left.\varphi=\left(\varphi_{1}, \ldots, \varphi_{k}\right):\right] 0, \epsilon\left[\rightarrow \mathbb{R}^{k} \backslash\{0\}\right.$ such that:

$$
\lim _{s \rightarrow 0}\|\phi(s)\|=\infty, \lim _{s \rightarrow 0}\|F(\phi(s))\|=\infty \text { and } \sum_{i=1}^{k} \varphi_{i}(s) \frac{\partial f_{i}}{\partial x_{l}}(\phi(s)) \equiv 0, \text { for } l=1, \ldots, n \text {. }
$$

We use notations, constructions and arguments as in the proof of the Theorem 4.3.8. The initial exponents of $\phi(s)$ as in (4.12) define the vectors $\mathbf{p}$ and $\mathbf{x}_{0}$. The assumption $\lim _{s \rightarrow 0}\|F(\phi(s))\|=\infty$ implies that there exists some $i$ such that $d_{\mathbf{p}}^{i}<0$. Then, as in the proof of Theorem 4.3.8, the face $\triangle_{\mathbf{p}}^{i}$ must be a face of $\Gamma^{+}\left(f_{i}\right)$ (see also Chen and Tibăr [8, Lemma 3.1]) and therefore one has $\mathrm{N}_{\mathbf{p}} \neq \emptyset$. From the condition $\phi(s) \subset \operatorname{Sing} F$ and comparing the orders of the expansions of the elements of (4.17), we obtain relations like (4.14) and (4.15). If we define $\mathrm{x}_{1}$ as in the proof of the Theorem 4.3.8 then these last relations imply $\mathrm{x}_{1} \in \operatorname{Sing} F_{\triangle_{\mathbf{p}}} \cap\left(\mathbb{R}^{*}\right)^{n}$, which contradicts the strongly non-degeneracy of $F$ (condition $(* *)$ of Definition 4.3.3). Thus, we show that $F(\operatorname{Sing} F) \cap\left(\mathbb{R}^{*}\right)^{k}$ not bounded 
implies a contradiction with the hypothesis of the strongly non-degeneracy of $F$. Therefore, we conclude that $F(\operatorname{Sing} F) \cap\left(\mathbb{R}^{*}\right)^{k}$ is bounded.

To show that $\mathcal{N}_{\infty}(F) \cap\left(\mathbb{R}^{*}\right)^{k}$ is bounded, we use Theorem 4.3.8, Remark 4.3.6 and the first step of the proof (i.e. the fact that $F(\operatorname{Sing} F) \cap\left(\mathbb{R}^{*}\right)^{k}$ is bounded). From Theorem 4.3.8, we have:

$$
\left(\mathcal{N}_{\infty}(F) \cap\left(\mathbb{R}^{*}\right)^{k}\right) \subset \underset{\triangle \in \mathscr{A}(F)}{\cup} F_{\triangle}\left(\operatorname{Sing} F_{\triangle} \cap\left(\mathbb{R}^{*}\right)^{n}\right) .
$$

On the other hand, by Remark 4.3.6, for any face $\triangle \in \mathscr{A}(F), F_{\triangle}$ is strongly nondegenerate at infinity and, from the first step of the proof, we have that if $F$ is strongly non-degenerate then $F(\operatorname{Sing} F) \cap\left(\mathbb{R}^{*}\right)^{k}$ is bounded. These imply that $F_{\triangle}\left(\operatorname{Sing} F_{\triangle}\right) \cap\left(\mathbb{R}^{*}\right)^{k}$ is bounded for any $\triangle \in \mathscr{A}(F)$. Since the set of faces $\triangle \in \mathscr{A}(F)$ is finite (see Remark 4.3.4), we conclude that $\underset{\triangle \in \mathscr{A}(F)}{\cup} F_{\triangle}\left(\operatorname{Sing} F_{\triangle} \cap\left(\mathbb{R}^{*}\right)^{n}\right)$ is bounded. This and the inclusion from (4.18) imply that $\mathcal{N}_{\infty}(F)$ is bounded, which ends our proof.

Corollary 4.3.11. Suppose that $F$ is non-degenerate at infinity and that $f_{i}$ is convenient, for any $i=1, \ldots, k$. Then $\mathcal{N}_{\infty}(F)=\emptyset$.

Proof. We use notations, constructions and arguments as in the proof of Theorem 4.3.8, but with the difference that $t$ is any point in $\mathbb{R}^{k}$, not only in $\left(\mathbb{R}^{*}\right)^{k}$. Since $f_{i}$ is convenient, we have $d_{\mathbf{p}}^{i}<0 \leq \operatorname{ord}_{t}\left(f_{i}(\phi(s))\right.$ for every $i$. Then, as in the proof of Theorem 4.3.8 and from [8, Lemma 3.1], the face $\triangle_{\mathbf{p}}^{i}$ must be a face of $\Gamma^{+}\left(f_{i}\right)$, for $i=1, \ldots, k$, which implies $\mathrm{N}_{\mathbf{p}}=\{1, \ldots, k\}$. As before, we obtain vector $\mathrm{x}_{0}, \mathrm{x}_{1}$ and equations like (4.14), (4.15), which imply $\mathrm{x}_{1} \in \operatorname{Sing} F_{\triangle_{\mathbf{p}}} \cap\left(\mathbb{R}^{*}\right)^{n}$. Since $d_{\mathbf{p}}^{i}<0$, we have $f_{\triangle_{\mathbf{p}}}\left(\mathrm{x}_{1}\right)=0$, for $i=1, \ldots, k$.

Therefore, we have $\mathrm{x}_{1} \in \operatorname{Sing} F_{\triangle_{\mathbf{p}}} \cap\left\{x \in \mathbb{R}^{n} \mid f_{\triangle_{\mathbf{p}}^{j}}(x)=0, \forall j \in \mathrm{N}_{\mathbf{p}}\right\} \cap\left(\mathbb{R}^{*}\right)^{n}$, which contradicts the non degeneracy of $F$ (condition $(*)$ of Definition 4.3.3).

Broughton [4, Proposition 3.4] showed that if a complex polynomial function $f: \mathbb{C}^{n} \rightarrow \mathbb{C}$ is convenient and Newton non-degenerate then $f$ is tame (Definition 2.2.6), which implies that $\mathcal{N}_{\infty}(f)=\emptyset$. Thus Corollary 4.3.11 extends to real mappings the Broughton's classical result.

Remark 4.3.12. We observe that results analogous to Theorem 4.3.8, Proposition 4.3.10 and Corollary 4.3.11 have been obtained by Chen and Tibăr [8] for mixed polynomial functions.

Remark 4.3.13. From Theorem 2.4.8 and Corollary 2.4.9, we may change in Proposition 4.3.10 and Corollary 4.3.11 the set $\mathcal{N}_{\infty}(F)$ with the set $\mathcal{N} \mathcal{T}_{\infty}(F)$.

\subsubsection{Non-degeneracy conditions at infinity}

\section{Bivià-Ausina non-degeneracy condition}

Bivià-Ausina [2] has formulated a Newton non-degeneracy condition for polynomial mappings $F: \mathbb{R}^{n} \rightarrow \mathbb{R}^{k}$ in order to study the Jacobian problem and Łojasiewicz exponents of $F$. 
His non-degeneracy condition is defined as follows:

Definition 4.3.14 (Bivià-Ausina [2, Definition 3.5]). The mapping $F: \mathbb{R}^{n} \rightarrow \mathbb{R}^{k}$ is nondegenerate at infinity if the following condition is satisfied for any $\mathbf{p}=\left(p_{1}, \ldots, p_{n}\right) \in \mathbb{Z}^{n}$ such that $p=\min _{1 \leq i \leq n} p_{i}<0$ :

$$
\left\{x \in\left(\mathbb{R}^{*}\right)^{n} \mid f_{\triangle_{\mathbf{p}}^{j}}(x)=0, \text { for all } j=1, \ldots, k\right\}=\emptyset .
$$

REMARK 4.3.15. In our constructions we have used the minimal value of the linear function $l_{\mathbf{p}}(v)=\sum_{i=1}^{n} p_{i} v_{i}$ on $\overline{\operatorname{supp}\left(f_{i}\right)}$, since we have considered analytic curves $\phi(s)$ depending on $s$ when $s \rightarrow 0$, while Bivià-Ausina used the maximal value of the linear function $l_{\mathbf{p}}(v)=$ $\sum_{i=1}^{n} p_{i} v_{i}$ on $\overline{\operatorname{supp}\left(f_{i}\right)}$ since he considered analytic curves $\phi(s)$ depending on $s$ when $s \rightarrow \infty$. Modulo this difference, the original definition of Bivià-Ausina [2] coincides to the Definition 4.3.14.

In next proposition, we see how Bivià-Ausina's definition of Newton non-degeneracy at infinity for mappings compares to our Definition 4.3.3.

Proposition 4.3.16. Let $F=\left(f_{1}, \ldots, f_{k}\right): \mathbb{R}^{n} \rightarrow \mathbb{R}^{k}, n \geq k$, be a polynomial mapping. Suppose that $f_{i}$ is convenient, for all $i=1, \ldots, k$. If $F$ is non-degenerate at infinity after Definition 4.3.14, then it is also non-degenerate at infinity after Definition 4.3.3. Moreover, if $k=n$ and $f_{i}$ is convenient, for all $i=1, \ldots, k$, the two definitions are equivalents.

Proof. We use the notations of 4.3.1 and 4.3.2. Firstly, we prove that non-degeneracy according to Definition 4.3.14 implies the non-degeneracy according to Definition 4.3.3. Thus, suppose that $F=\left(f_{1}, \ldots, f_{k}\right)$ is non-degenerate in the sense of 4.3 .14 and that $f_{i}$ is convenient, for all $i=1, \ldots, k$. Let $\mathbf{p}=\left(p_{1}, \ldots, p_{n}\right)$ be a vector in $\mathbb{Z}^{n}$ with $p=\min _{1 \leq i \leq n}\left\{p_{i}\right\}<0$. Since $f_{i}$ is convenient for any $i=1, \ldots, k$, the minimal value $d_{\mathbf{p}}^{i}$ of $l_{\mathbf{p}}$ on $\overline{\operatorname{supp}\left(f_{i}\right)}$ must be strictly negative, for any $i$. Therefore, for any $i, \triangle_{\mathbf{p}}^{i}$ must be a face of $\Gamma^{+}\left(f_{i}\right)$ and consequently $\mathrm{N}_{\mathbf{p}}=\{1, \ldots, k\}$. From this last equality and since we have assumed that $F$ satisfies the condition (4.19), we obtain that $F$ satisfies the condition $(*)$ of Definition 4.3 .3 and therefore $F$ is non-degenerate after Definition 4.3.3.

In the case $n=k$, let us assume that $F$ is degenerate at infinity under Definition 4.3.14. Thus, there exists $\mathrm{x}_{1} \in\left(\mathbb{R}^{*}\right)^{n}$ and a vector $\mathbf{p}=\left(p_{1}, \ldots, p_{n}\right) \in \mathbb{Z}^{n} \backslash\{0\}$ with $\min _{1 \leq i \leq n} p_{i}<0$ such that, as before, one has $\triangle_{\mathbf{p}}^{i} \in \Gamma^{+}\left(f_{i}\right)$ and $f_{\triangle_{\mathbf{p}}}\left(\mathrm{x}_{1}\right)=0$, for $i=1, \ldots, k$, which imply that

$$
\mathrm{x}_{1} \in\left\{x \in\left(\mathbb{R}^{*}\right)^{n} \mid f_{\triangle_{\mathbf{p}}^{i}}(x)=0, \forall i \in \mathrm{N}_{\mathbf{p}}\right\}, \text { where } \mathrm{N}_{\mathbf{p}}=\{1, \ldots, k\} .
$$

From Euler's identity, if we denote $\mathbf{p x}_{1}:=\left(p_{1} x_{1}, \ldots, p_{n} x_{n}\right)$ then we have the following equalities $\left\langle\nabla f_{\triangle_{\mathbf{p}}^{i}}\left(\mathrm{x}_{1}\right), \mathbf{p x}_{1}\right\rangle=d_{\mathbf{p}}^{i} f_{\triangle_{\mathbf{p}}^{i}}\left(\mathrm{x}_{1}\right)=0$, for $i=1, \ldots, n$, where the last equality follows by (4.20) . These equalities imply that the Jacobian matrix of $F_{\triangle_{\mathbf{p}}}$ at $\mathrm{x}_{1}$ evaluated at $\mathbf{p x} \mathbf{x}_{1}$ is equal to zero, i.e. $\mathrm{D} F\left(\mathrm{x}_{1}\right) \mathbf{p x}_{1}=0$, which gives $\mathrm{x}_{1} \in \operatorname{Sing} F_{\triangle_{\mathbf{p}}}$. This and (4.20) imply that $F$ 
is degenerate after Definition 4.3.3. This and the first step of our proof show the equivalence of the two definitions in the case $n=k$.

We point out that the first implication in the above proposition is not an equivalence in general, i.e., in general there exist maps $F$, where each $f_{i}$ is convenient, so that $F$ is non-degenerate after Definition 4.3.3 and $F$ is degenerate after Definition 4.3.14 (for example $\left.f\left(x_{1}, x_{2}\right)=x_{1}^{2}-x_{2}^{2}\right)$.

\section{Examples}

In this subsection we present examples to illustrate the results of $\S 4.3$. In particular, the first two examples show that if we remove in Proposition 4.3.16 the condition that $f_{i}$ is convenient, for $i=1, \ldots, k$, then there are examples where Definition 4.3 .3 does not imply Definition 4.3.14 (Example 4.3.17), and there are examples where Definition 4.3.14 does not imply Definition 4.3.3 (Example 4.3.18).

Example 4.3.17. Consider $F: \mathbb{R}^{3} \rightarrow \mathbb{R}^{2}, F\left(x_{1}, x_{2}, x_{3}\right)=\left(x_{1}+x_{2}, x_{2}^{2}-x_{3}^{2}\right)$. We show that $F$ is non-degenerate at infinity in the sense of Definition 4.3.3, but degenerate in the sense of Definition 4.3.14. We have $\operatorname{Sing} F=\left\{\left(x_{1}, x_{2}, x_{3}\right) \in \mathbb{R}^{3} \mid x_{2}=x_{3}=0\right\}$, so $F(\operatorname{Sing} F)=$ $\left\{t=\left(t_{1}, 0\right) \mid t_{1} \in \mathbb{R}\right\}$ and we also have $\mathcal{N}_{\infty}(F)=\emptyset$.

First, we show that $F$ is non-degenerate after Definition 4.3.3 and that $F$ is degenerate after Definition 4.3.14:

Let $\mathbf{p}=\left(p_{1}, p_{2}, p_{3}\right) \in \mathbb{Z}^{3}$ such that $p=\min _{1 \leq i \leq 3} p_{i}<0$. From 4.3.4, the cardinality of the set $\left\{F_{\triangle_{\mathbf{p}}} \mid \mathbf{p} \in \mathbb{Z}^{n} \backslash 0\right\}$ is at most 9 . Describing in more detail, one has the following possibilities:

Table 4.1: Possibilities for $F_{\triangle_{\mathbf{p}}}$

\begin{tabular}{||l|c||}
\hline \hline Faces defined by $\mathbf{p}$ & Conditions about the vector $\mathbf{p}$ \\
\hline$F_{\triangle_{1}}=\left(x_{1},-x_{3}^{2}\right)$ & $p_{3}<p_{1}<p_{2} ;$ or $p_{1}<p_{3}<p_{2} ;$ or $p_{1}=p_{3}<p_{2}$ \\
\hline$F_{\triangle_{2}}=\left(x_{2},-x_{3}^{2}\right)$ & $p_{3}<p_{2}<p_{1}$ \\
\hline$F_{\triangle_{3}}=\left(x_{1}+x_{2},-x_{3}^{2}\right)$ & $p_{3}<p_{1}=p_{2}$ \\
\hline$F_{\triangle_{4}}=\left(x_{2}, x_{2}^{2}\right)$ & $p_{2}<p_{1}<p_{3} ;$ or $p_{2}<p_{3}<p_{1} ;$ or $p_{2}<p_{1}=p_{3}$ \\
\hline$F_{\triangle_{5}}=\left(x_{1}+x_{2}, x_{2}^{2}\right)$ & $p_{1}=p_{2}<p_{3}$ \\
\hline$F_{\triangle_{6}}=\left(x_{1}+x_{2}, x_{2}^{2}-x_{3}^{2}\right)$ & $p_{1}=p_{2}=p_{3}$ \\
\hline$F_{\triangle_{7}}=\left(x_{2}, x_{2}^{2}-x_{3}^{2}\right)$ & $p_{2}=p_{3}<p_{1}$ \\
\hline$F_{\triangle_{8}}=\left(x_{1}, x_{2}^{2}\right)$ & $p_{1}<p_{2}<p_{3}$ \\
\hline$F_{\triangle_{9}}=\left(x_{1}, x_{2}^{2}-x_{3}^{2}\right)$ & $p_{1}<p_{2}=p_{3}$ \\
\hline
\end{tabular}

(a). $F_{\triangle_{i}}$, for $i=1,2,3,5,7,8$. The Jacobian matrix of $\mathrm{D} F_{\triangle_{i}}$, for $i=1,2,3,7$, has not maximal rank if and only if $x_{3}=0$; and the Jacobian matrix of $\mathrm{D} F_{\triangle_{i}}$, for $i=5,8$, has not maximal rank if and only if $x_{2}=0$. Then the strong non-degeneracy condition at infinity is verified for $F_{\triangle_{i}}, i=1,2,3,5,7,8$. Since the strong non-degeneracy condition implies the 
non-degeneracy condition, we conclude that the restrictions $F_{\triangle_{i}}$, for $i=1,2,3,5,7,8$, verify the non-degeneracy condition.

(b). $F_{\triangle_{i}}$, for $i=6,9$. The Jacobian matrix of $\mathrm{D} F_{\triangle_{i}}$, for $i=6,9$, has not maximal rank if and only if $x_{2}=x_{3}=0$. This implies that the strong non-degeneracy condition at infinity is verified for $F_{\triangle_{i}}, i=6,9$. Since the strong non-degeneracy condition implies the non-degeneracy condition, we conclude that the restrictions $F_{\triangle_{i}}, i=6,9$, verify the non-degeneracy condition.

(c). The case $F_{\triangle_{4}}$. From the conditions on $\mathbf{p}$ to define $F_{\triangle_{4}}$ in Table 4.1 and since we have supposed $\min _{1 \leq i \leq 3} p_{i}<0$, we obtain that $p=p_{2}<0$, which implies that $\mathrm{N}_{\mathbf{p}}=\{1,2\}$. Thus, since we have $\operatorname{Sing} F_{\triangle_{4}}=\mathbb{R}^{3}$ and $\operatorname{Sing} F_{\triangle_{4}} \cap\left\{F_{\triangle_{4}}=0\right\} \cap\left(\mathbb{R}^{*}\right)^{3}=\emptyset$, we conclude that $F_{\triangle_{4}}$ is strongly degenerate at infinity but $F_{\triangle_{4}}$ satisfies the non-degenerate at infinity.

To finish, since we have shown that $F$ satisfies the condition $(*)$ of Definition 4.3.3 in the nine cases of Table 4.1, we conclude that $F$ is non-degenerate after Definition 4.3.3.

Secondly, we will show that $F$ is degenerate after Definition 4.3.14. In order to show this, we consider the restriction $F_{\triangle_{6}}$ (note that if we take $\mathbf{p}=(-1,-1,-1)$ then $F_{\triangle_{\mathbf{p}}}=F_{\triangle_{6}}$, see also Table 4.1). the equation $F_{\triangle_{6}}=0$ has solution in $\left(\mathbb{R}^{*}\right)^{3}\left(x_{1}=-x_{2}=x_{3}\right.$ or $x_{1}=-x_{2}=$ $\left.-x_{3}\right)$. Therefore $F$ does not satisfy the non-degeneracy condition in the sense of Definition 4.3.14.

Now, we compute $F(\operatorname{Sing} F)$ and $\mathcal{N}_{\infty}(F)$. The Jacobian matrix of $F$ is

$$
\left(\begin{array}{ccc}
1 & 1 & 0 \\
0 & 2 x_{2} & -2 x_{3}
\end{array}\right),
$$

which implies that $\operatorname{Sing} F=\left\{\left(x_{1}, 0,0\right) \mid x_{1} \in \mathbb{R}\right\}$ and $F(\operatorname{Sing} F)=\left\{\left(t_{1}, 0\right) \mid t_{1} \in \mathbb{R}\right\}$.

We now show that $\mathcal{N}_{\infty}(F)=\emptyset$ by using the Rabier function (see Defintion 2.3.1). From the definition of the Rabier function (Definition 1.3.1), one has:

$$
\nu\left(\operatorname{DF}\left(x_{1}, x_{2}, x_{3}\right)\right)=\min \left\{\left\|\left(\varphi_{1}, \varphi_{1}+2 \varphi_{2} x_{2},-2 \varphi_{2} x_{3}\right)\right\| \mid\left(\varphi_{1}, \varphi_{2}\right) \in \mathbb{R}^{2} \text { and } \varphi_{1}^{2}+\varphi_{2}^{2}=1\right\}
$$

Suppose that $\left(t_{1}, t_{2}\right) \in \mathcal{N}_{\infty}(F)$. Then by Curve Selection Lemma (Milnor [34, §3]), there exist curves $\phi(s)=\left(\phi_{1}(s), \phi_{2}(s), \phi_{3}(s)\right)$ and $\varphi(s)=\left(\varphi_{1}(s), \varphi_{2}(s)\right)$ such that:

$$
\begin{gathered}
\lim _{s \rightarrow 0}\|\phi(s)\|=\infty, \\
\lim _{s \rightarrow 0} F(\phi(s))=\lim _{s \rightarrow 0}\left(\phi_{1}(s)+\phi_{2}(s), \phi_{2}^{2}(s)-\phi_{3}^{2}(s)\right)=\left(t_{1}, t_{2}\right), \\
\lim _{s \rightarrow 0}\|\phi(s)\| \nu(\mathrm{D} F(\phi(s)))=\lim _{s \rightarrow 0}\|\phi(s)\|\left\|\left(\varphi_{1}(s), \varphi_{1}(s)+2 \varphi_{2}(s) \phi_{2}(s),-2 \varphi_{2}(s) \phi_{3}(s)\right)\right\|=0,
\end{gathered}
$$

where $\varphi_{1}^{2}(s)+\varphi_{2}^{2}(s)=1$. From (4.23) and (4.25), we obtain $\lim _{s \rightarrow 0} \nu(\mathrm{D} F(\phi(s)))=0$. This and (4.22) impliy $\lim _{s \rightarrow 0} \varphi_{1}(s)=0$ and, since $\varphi_{1}^{2}(s)+\varphi_{2}^{2}(s)=1$, we also obtain $\lim _{s \rightarrow 0} \varphi_{2}(s)= \pm 1$. 
These and $\lim _{s \rightarrow 0} \nu(\mathrm{D} F(\phi(s)))=0$ imply $\lim _{s \rightarrow 0} \phi_{2}(s)=0$ and $\lim _{s \rightarrow 0} \phi_{3}(s)=0$. These two last limits and (4.23) imply $\lim _{s \rightarrow 0}\left\|\phi_{1}(s)\right\|=\infty$.

Thus, we have obtained the $\operatorname{limits}_{\lim _{s \rightarrow 0}} \phi_{2}(s)=0$ and $\lim _{s \rightarrow 0}\left\|\phi_{1}(s)\right\|=\infty$, which imply $\lim _{s \rightarrow 0}\left\|\phi_{1}(s)+\phi_{2}(s)\right\|=\infty$. But this last limit gives a contradiction with the equality (4.24) and we therefore conclude that $\mathcal{N}_{\infty}(F)=\emptyset$.

To finish, we compute the fibres of $F$. For any critical value $\left(t_{1}, 0\right)$, we have:

$$
F^{-1}\left(\left(t_{1}, 0\right)\right)=\left\{\left(t_{1}-x_{2}, x_{2}, x_{2}\right) \mid y \in \mathbb{R}\right\} \cup\left\{\left(t_{1}-x_{2}, x_{2},-x_{2}\right) \mid x_{2} \in \mathbb{R}\right\},
$$

which is the union of two lines and these two lines intersect at the point $\left(t_{1}, 0,0\right)$.

If we fix $\varepsilon<0$, then the regular fibre of $\left(t_{1}, \varepsilon\right)$ is:

$$
F^{-1}\left(\left(t_{1}, \varepsilon\right)\right)=\left\{\left(x_{1}, x_{2}, x_{3}\right) \in \mathbb{R}^{3} \mid x_{1}=t_{1}-x_{2}, x_{3}= \pm \sqrt{x_{2}^{2}-\varepsilon}\right\} .
$$

Therefore the regular fibre of $\left(t_{1}, \varepsilon\right)$ is a hyperbolic curve and moreover, the asymptotes of this hyperbolic curve are the lines of $F^{-1}\left(\left(t_{1}, 0\right)\right)$. If we fix $\varepsilon>0$, the regular fiber of $\left(t_{1}, \varepsilon\right)$ is given by:

$$
F^{-1}\left(\left(t_{1}, \varepsilon\right)\right)=\left\{\left(t_{1}-x_{2}, x_{2}, \pm \sqrt{x_{2}^{2}-\varepsilon}\right) \mid x_{2} \leq-\sqrt{\varepsilon} \text { or } \sqrt{\varepsilon} \leq x_{2}\right\} .
$$

Example 4.3.18. Let $F=\left(f_{1}, f_{2}\right): \mathbb{R}^{2} \rightarrow \mathbb{R}^{2}$ be a polynomial mapping defined by $F\left(x_{1}, x_{2}\right)=$ $\left(x_{1}-x_{1} x_{2}^{2}, x_{2}\right)$. Then $F$ is non-degenerate in the sense of Definition 4.3.14, but $F$ is degenerate in the sense of Definition 4.3.3.

The non-degenerate in the sense of Definition 4.3 .14 follows by the fact that $f_{\triangle_{\mathbf{p}}^{2}}=x_{2}$, for any $\mathbf{p} \in \mathbb{Z}^{2}$, which implies $\left\{F_{\triangle_{\mathbf{p}}}=0\right\} \cap\left(\mathbb{R}^{*}\right)^{2}=\emptyset$. Consequently the condition (4.19) is satisfied for any $\mathbf{p} \in \mathbb{Z}^{2}$, which shows that $F$ is non-degenerate after the Definition (4.3.14).

On the other hand, if we consider $\mathbf{p}=(-1,0)$ then $F_{\triangle_{\mathbf{p}}}=F, \mathrm{~N}_{\mathbf{p}}=\{1\}$, and $\operatorname{Sing} F_{\triangle_{\mathbf{p}}}=$ $\left\{\left(x_{1}, \pm 1\right) \mid x_{1} \in \mathbb{R}\right\}$. So, for $\lambda \neq 0$, we have $\left.(\lambda, \pm 1) \in \operatorname{Sing} F_{\triangle_{\mathbf{p}}}\right) \cap\left\{\left(x_{1}, x_{2}\right) \in \mathbb{R}^{2} \mid\right.$ $\left.f_{\triangle 1}\left(x_{1}, x_{2}\right)=0\right\} \cap\left(\mathbb{R}^{*}\right)^{2}$. This show that $F$ is degenerate in the sense of Definition 4.3.3.

In next example, we consider a non-degenerate (Definition 4.3.3) and convenient polynomial mapping $F: \mathbb{R}^{2} \rightarrow \mathbb{R}^{2}$. Thus, we may use the Corollary 4.3.11 to conclude that $\mathcal{N}_{\infty}(F)=\emptyset$.

ExAmple 4.3.19 (see also Example 5.2.4). Let $F=\left(f_{1}, f_{2}\right): \mathbb{R}^{2} \rightarrow \mathbb{R}^{2}$ be a polynomial mapping defined by $F\left(x_{1}, x_{2}\right)=\left(x_{1}+x_{2}+x_{1} x_{2}^{2}, x_{1}+2 x_{2}+x_{1} x_{2}^{2}\right)$. Then $f_{1}, f_{2}$ are convenient, $F$ is Newton non-degenerate and $F(\operatorname{Sing} F)=\emptyset$. Consequently, by Corollary 4.3.11, one has $\mathcal{N}_{\infty}(F)=\emptyset$.

EXAmple 4.3.20. Let $F: \mathbb{R}^{2} \rightarrow \mathbb{R}^{2}$ be a polynomial mapping defined by $F\left(x_{1}, x_{2}\right)=\left(x_{1}^{2}-\right.$ $\left.x_{1} x_{2}+x_{2}^{3}, x_{1}^{2}-x_{1} x_{2}+2 x_{2}^{3}\right)$. Then $f_{1}, f_{2}$ are convenient and $F$ is non-degenerate (Definition 
4.3.3). Thus, we may use the Corollary 4.3 .11 to conclude that $\mathcal{N}_{\infty}(F)=\emptyset$.

Let $\mathbf{p} \in \mathbb{Z}^{2}$. We observe that, since the polyhedrons of $f_{1}$ and $f_{2}$ are the same, the vector $\mathbf{p}$ defines the same face in $\overline{\operatorname{supp}\left(f_{1}\right)}$ and in $\overline{\operatorname{supp}\left(f_{2}\right)}$. Moreover, one has $d_{\mathbf{p}}^{1}=d_{\mathbf{p}}^{2}$ and consequently either $\mathrm{N}_{\mathbf{p}}=\{1,2\}$ or $\mathrm{N}_{\mathbf{p}}=\emptyset$. Thus, since $\mathbf{p}$ defines the same face in $f_{1}$ and $f_{2}$, we have the following possibilities for $F_{\triangle_{\mathbf{p}}}: F_{\triangle_{1}}=\left(x_{1}^{2}, x_{1}^{2}\right), F_{\triangle_{2}}=\left(-x_{1} x_{2},-x_{1} x_{2}\right)$, $F_{\triangle_{3}}=\left(x_{2}^{3}, x_{2}^{3}\right), F_{\triangle_{4}}=\left(x_{1}^{2}-x_{1} x_{2}, x_{1}^{2}-x_{1} x_{2}\right), F_{\triangle_{5}}=\left(x_{1}^{2}+x_{2}^{3}, x_{1}^{2}+2 x_{2}^{3}\right), F_{\triangle_{6}}=\left(-x_{1} x_{2}+\right.$ $\left.x_{2}^{3},-x_{1} x_{2}+2 x_{2}^{3}\right)$ an $F_{\triangle_{7}}=F$. Therefore, in order to show that $F$ is non-degenerate (see Definition 4.3.3), we need only to show that, for any $i \in\{1, \ldots, 7\}, F_{\triangle_{i}}$ satisfies the condition (*) of Definition 4.3.3.

The case $F_{\triangle_{i}}$, for $i=1,2,3$, verifies the condition $(*)$ of Definition 4.3 .3 , since $\left\{F_{\triangle_{i}}=\right.$ $0\} \cap\left(\mathbb{R}^{*}\right)^{2}=\emptyset$.

Let $\mathbf{p}$ be a vector that defines $F_{\triangle_{4}}$. From the equations of $F_{\triangle_{4}}$ one has $2 p_{1}=p_{1}+p_{2}<3 p_{2}$, which implies $0<p_{1}=p_{2}$. This implies $d_{\mathbf{p}}^{j}>0, j=1,2$, and consequently $\mathrm{N}_{\mathbf{p}}=\emptyset$. So, we do not need to consider this face.

In the cases $F_{\triangle_{i}}$, for $i=5,6,7$, one has that $\left\{F_{\triangle_{i}}=0\right\}=\{(0,0)\}$, which implies $\left\{F_{\triangle_{i}}=0\right\} \cap\left(\mathbb{R}^{*}\right)^{2}=\emptyset$. Therefore the condition $(*)$ of Definition 4.3.3 is satisfied.

Therefore, we have shown that, for any $i \in\{1, \ldots, 7\}, F_{\triangle_{i}}$ satisfies the condition $(*)$ of Definition 4.3.3, we have that $F$ is non-degenerate. Moreover, since $f_{1}, f_{2}$ are convenient, we may use the Corollary 4.3.11 to conclude that $\mathcal{N}_{\infty}(F)=\emptyset$.

\section{Khovanskii non-degeneracy condition}

Motivated by the definition of Khovanskii in [26] we have:

Definition 4.3.21 ([26, page 291]). Let $F: \mathbb{R}^{n} \rightarrow \mathbb{R}^{k}$ be a polynomial mapping. We say that $F$ is non-degenerate if for any vector $\mathbf{p} \in \mathbb{Z}^{n}$ one has:

$$
\operatorname{Sing} F_{\triangle_{\mathbf{p}}} \cap\left\{F_{\triangle_{\mathbf{p}}}=0\right\} \cap\left(\mathbb{R}^{*}\right)^{n}=\emptyset
$$

The two next examples show that Definition 4.3.3 does not imply Definition 4.3.21, and vice versa.

EXAmple 4.3.22. Let $F=\left(f_{1}, f_{2}\right): \mathbb{R}^{2} \rightarrow \mathbb{R}^{2}$ be as in Example 4.3.18, i.e. $F\left(x_{1}, y\right)=$ $\left(x_{1}-x_{1} x_{2}^{2}, x_{2}\right)$. Then $F$ is non-degenerate in the sense of Definition 4.3.21, but $F$ is degenerate in the sense of Definition 4.3.3.

The degeneracy of $F$ after Definition 4.3.3 was shown in Example 4.3.18. Thus, we will show here only the non-degeneracy of $F$ in the sense of Definition 4.3.21. For any $\mathbf{p} \in \mathbb{Z}^{2}$, one has $f_{\triangle_{\mathbf{p}}^{2}}=x_{2}$. This implies $\left\{F_{\triangle_{\mathbf{p}}}=0\right\} \cap\left(\mathbb{R}^{*}\right)^{2}=\emptyset$, which implies the condition 4.29 and therefore we have that $F$ is non-degenerate after Definition 4.3.21. 
EXAmple 4.3.23. Let $F: \mathbb{R}^{2} \rightarrow \mathbb{R}^{2}$ be as in Example 4.3.20, i.e. $F\left(x_{1}, x_{2}\right)=\left(x_{1}^{2}-x_{1} x_{2}+\right.$ $\left.x_{2}^{3}, x_{1}^{2}-x_{1} x_{2}+2 x_{2}^{3}\right)$. Then $F$ is non-degenerate in the sense of Definition 4.3 .3 but $F$ is degenerate in the sense of Definition 4.3.21.

That $F$ is non-degenerate after Definition 4.3.3 was shown in Example 4.3.20. Thus, we need only to show that $F$ is degenerate in the sense of Definition 4.3.21. Let $\mathbf{p}=(1,1)$. Then $F_{\triangle_{\mathbf{p}}}=\left(x^{2}-x y, x^{2}-x y\right)$ and one has the following equalities: $\operatorname{Sing} F_{\triangle_{\mathbf{p}}}=\mathbb{R}^{2},\left\{F_{\triangle_{\mathbf{p}}}=0\right\}=$ $\{(x, y) \mid x(x-y)=0\}$. These implies that $\left\{(\lambda, \lambda) \mid \lambda \in \mathbb{R}^{*}\right\} \subset \operatorname{Sing} F_{\triangle_{\mathbf{p}}} \cap\left\{F_{\triangle_{\mathbf{p}}}=0\right\} \cap\left(\mathbb{R}^{*}\right)^{2}$, which implies the degeneracy of $F_{\triangle_{\mathbf{p}}}$ and consequently the degeneracy of $F$ in the sense of Definition 4.3.21. 


\title{
Some consequences
}

\author{
Contents \\ 5.1 Relative Case . . . . . . . . . . . . . . . . . . 70

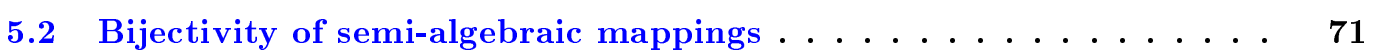 \\ $5.3 \quad$ Euler characteristic $\ldots \ldots \ldots \ldots \ldots \ldots 74$
}

\section{Brief Resume}

We discuss some consequences from the previous chapters. In $§ 5.1$, we follow Jelonek [25] to define in a local point of view (like Definition 2.4.1) Rabier, Jelonek, Gaffney and Kuo-KOS conditions for mappings $f: X \rightarrow \mathbb{K}^{p}$, where $X \subset \mathbb{K}^{n}$ is a smooth affine variety. Then, we discuss Theorem 2.4.8 for these mappings (Proposition 5.1.3).

In $\S 5.2$, we discuss the problem of bijectivity of semi-algebraic mappings. As consequence of this study and results of $\S 4.3$, we prove that if a polynomial mapping $f=\left(f_{1}, \ldots, f_{n}\right): \mathbb{R}^{n} \rightarrow$ $\mathbb{R}^{n}$ is Newton non-degenerate at infinity, $f_{i}$ is convenient for all $i=1, \ldots, n$, and $\operatorname{Sing} f=\emptyset$ then $f$ is a global diffeomorphism.

To finish, we present in $\S 5.3$ a formula to compute the Euler characteristic of regular fibres of polynomial mappings $f: \mathbb{R}^{n} \rightarrow \mathbb{R}^{n-1}$. 


\section{$5.1 \quad$ Relative Case}

In this section, we consider a smooth affine variety $X \subset \mathbb{K}^{n}(\mathbb{K}=\mathbb{R}, \mathbb{C})$ and we suppose that $X$ is a global complete intersection. In other words $X=\left\{x \in \mathbb{K}^{n} \mid h_{1}(x)=h_{2}(x)=\right.$ $\left.\ldots=h_{r}(x)=0\right\}$ and $\operatorname{rank} \mathrm{D} h(x)=r$ for any $x \in X$, where $h=\left(h_{1}, \ldots, h_{r}\right): \mathbb{K}^{n} \rightarrow \mathbb{K}^{r}$ is a polynomial mapping. With the functions of $\S 1.4$ and as in Definition 2.3.1, we have:

Definition 5.1.1. Let $f: X \rightarrow \mathbb{K}^{p}$ be the restriction of a $C^{1}$ mapping to $X$, with $\operatorname{dim} X \geq p$. We define:

$$
\begin{aligned}
\mathcal{N}_{\infty}(f):= & \left\{t \in \mathbb{K}^{p} \mid \exists\left\{x_{j}\right\}_{j \in \mathbb{N}} \subset X, \lim _{j \rightarrow \infty}\left\|x_{j}\right\|=\infty\right. \\
& \left.\lim _{j \rightarrow \infty} f\left(x_{j}\right)=t \text { and } \lim _{j \rightarrow \infty}\left\|x_{j}\right\| \beta\left(\mathrm{D} f\left(x_{j}\right), T_{x_{j}} X\right)=0\right\},
\end{aligned}
$$

where $\mathrm{D} f(x)$ denotes the Jacobian of $f$ at $x$; and $\beta$ stands for relative Rabier function $\nu$ (Definition 1.4.1), relative Kuo function $\kappa$ (Definition 1.4.3), relative Gaffney function $\gamma$ (Definition 1.4.4), relative Jelonek function (Definition 1.4.5).

Jelonek [25] considered the case of a smooth mapping $f: X \rightarrow \mathbb{K}^{p}, \operatorname{dim} X \geq p$, and proved that $B(f) \subset\left(f(\operatorname{Sing} f) \cup \mathcal{N}_{\infty}(f)\right)$. On the other hand, we have obtained in Theorem 2.4.8 and Corollary 2.4.9, an equivalence between $t$-regularity and Rabier condition (or Gaffney, Jelonek, Kuo-KOS conditions) for $C^{1}$ semi-algebraic mappings $f: \mathbb{R}^{n} \rightarrow \mathbb{R}^{p}$ and for complex polynomial mappings $f: \mathbb{C}^{n} \rightarrow \mathbb{C}^{p}$. These equivalences motivated the next definition and proposition.

Definition 5.1.2. Let $f: X \rightarrow \mathbb{K}^{p}$ be a polynomial mapping, with $\operatorname{dim} X \geq p$. We say that $z_{0} \in \mathbb{X}^{\infty}$ satisfies Rabier condition (respectively Gaffney condition, Jelonek condition and Kuo-KOS condition) when one uses the relative Rabier function $\nu$ (respectively relatives functions of Gaffney, Jelonek and Kuo) to generate $\mathcal{N}_{\infty}(f)$ and $z_{0} \notin\left(\tau^{-1}\left(\mathcal{N}_{\infty}(f)\right) \cap \mathbb{X}^{\infty}\right)$, where $\mathbb{X}^{\infty}$ and $\tau$ are as in subsection "Notation (page 27 )".

Proposition 5.1.3. Let $f: X \rightarrow \mathbb{K}^{p}$ be the restriction of a polynomial mapping to $X$, with $\operatorname{dim} X \geq p$. Let $z_{0} \in \mathbb{X}^{\infty}$. Then $f$ is t-regular at $z_{0}$ if and only if $f$ satisfies Rabier condition (equivalently Gaffney, Jelonek and Kuo-KOS conditions) at $z_{0}$.

Proof. The proof is similar to the proof of Theorem 2.4.8 and can be obtained as follows: firstly, we obtain an interpretation to $t$-regularity in terms of normal vectors to $X$ and to the fibres of $f$ in $X$ (like subsection " $t$-regularity interpretation (page 30)"). Then, with this interpretation and the function $\nu_{1}$ presented in Lemma 1.4.2, which is equivalent to the relative function of Rabier, the proof follows as in Theorem 2.4.8.

REMARK 5.1.4. In the above proposition, we suppose that $X \subset \mathbb{K}^{n}$ is a complete intersection but it is possible to give this result in the general case of a smooth affine variety $X$. In fact, since any manifold is locally a complete intersection (see for instance [21, Exercise 2, page 
18]), we may take a locally finite cover $\mathcal{V}:=\left\{V_{i}\right\}$ of $\mathbb{K}^{n}$ such that the manifold $X_{i}:=X \cap V_{i}$ is a complete intersection. Then we consider the normal vector fields on each $X_{i}$ as in " $t$ regularity interpretation (page 30)" and use a partition of unity subordinate to the cover $\mathcal{V}$ to obtain normal vector fields defined on $X$ and the proof in this case follows as in Theorem 2.4.8.

\subsection{Bijectivity of semi-algebraic mappings}

There is a large literature concerning bijectivity of mappings (see for instance the book [58] for references and related problems). In this section, we discuss a little about bijectivity of semi-algebraic mappings $f: \mathbb{R}^{n} \rightarrow \mathbb{R}^{n}$ and its relation with our work.

We start with the following evident fact: let $f=\left(f_{1}, \ldots, f_{n}\right): \mathbb{R}^{n} \rightarrow \mathbb{R}^{n}$ be a $C^{1}$ diffeomorphism, i.e. $\exists$ a $C^{1}$ mapping $h: \mathbb{R}^{n} \rightarrow \mathbb{R}^{n}$ such that $f \circ h=h \circ f=\operatorname{Id}_{\mathrm{n}}$, where $\operatorname{Id}_{\mathrm{n}}$ stands for the identity mapping of $\mathbb{R}^{n}$. Then $B\left(f_{I}\right)=\emptyset$, for any multi-index $I=\left(i_{1}, \ldots, i_{j}\right) \subset\{1, \ldots, n\}$, $1 \leq j \leq n$ and $f_{I}=\left(f_{i_{1}}, \ldots, f_{i_{j}}\right)$. This follows by the fact that, after the change of coordinates by $h$ in $\mathbb{R}^{n}$, we have that $f_{I}$ is just a projection.

The following criterion holds:

Proposition 5.2.1. Let $f: \mathbb{R}^{n} \rightarrow \mathbb{R}^{n}$ be a $C^{1}$ real semi-algebraic mapping such that $\operatorname{Sing} f=$ $\emptyset$. If $\mathcal{N}_{\infty}(f)=\emptyset^{1}$ then $f$ is a global diffeomorphism.

In particular, if $f=\left(f_{1}, \ldots, f_{n}\right)$ is a polynomial mapping, non-degenerate at infinity, Sing $f=\emptyset$ and if $f_{i}$ is convenient for all $i=1, \ldots, n$ then $f$ is a global diffeomorphism.

Proof. Let $J_{f}$ be the set of points at which $f$ is not proper (see definition below). By Kurdyka, Orro, Simon [29, Proposition 3.1], we have $\mathcal{N}_{\infty}(f)=J_{f}$. Thus if $\mathcal{N}_{\infty}(f)=\emptyset$ then $f$ is proper. It is moreover a submersion $\operatorname{since} \operatorname{Sing} F=\emptyset$ by hypothesis. A proper submersion is an open and closed mapping, a general topological fact. Thus $f$ is a covering and it must be one-to-one since its image $\mathbb{R}^{n}$ is simply connected. Our first assertion follows. Remark that the final part of this proof is actually Hadamard's theorem (see e.g. van den Essen [58, Theorem 10.1.1]). The second assertion follows by Corollary 4.3.11.

Bivià-Ausina [2] proved the second statement for polynomial mappings $f: \mathbb{R}^{n} \rightarrow \mathbb{R}^{n}$. He obtains the properness of $f$, necessary for the bijectivity of $f$, via an interpretation of his notion of non-degeneracy in terms of Łojasiewicz exponents.

Definition 5.2.2 (Jelonek [25, Definition 3.3]). Let $f: \mathbb{K}^{n} \rightarrow \mathbb{K}^{p}$ be a continuous map. We say that $f$ is proper at a point $t \in \mathbb{R}^{p}$ if there exists an open neighborhood $U$ of $t$ such that the restriction $f_{\mid f^{-1}(U)}: f^{-1}(U) \rightarrow U$ is a proper map. We denote by $J_{f}$ the set of points at which $f$ is not proper.

\footnotetext{
${ }^{1}$ or $\mathcal{N} \mathcal{T}_{\infty}(f)=\emptyset$ by Corollary 2.4.9.
} 
EXAmpLe 5.2.3. Let $F: \mathbb{R}^{2} \rightarrow \mathbb{R}^{2}$ be the polynomial mapping defined in Example 4.3.20. The functions $f_{1}, f_{2}$ are convenient and we have that $F$ is Newton non-degenerate. From Corollary 4.3.11, these imply $\mathcal{N}_{\infty}(F)=\emptyset$. However, $F(\operatorname{Sing} F) \neq \emptyset$ and $F$ is not invertible. Therefore, this example shows that we cannot eliminate the hypothesis $\operatorname{Sing} F=\emptyset$ in Proposition 5.2.1, in other worlds, $F$ Newton non-degenerate and $f_{i}$ convenient for all $i$ do not imply $\operatorname{Sing} F=\emptyset$.

EXAmple 5.2.4. Let $F=\left(f_{1}, f_{2}\right): \mathbb{R}^{2} \rightarrow \mathbb{R}^{2}$ be the polynomial mapping as in Example 4.3.19, i.e., $F\left(x_{1}, x_{2}\right)=\left(x_{1}+x_{2}+x_{1} x_{2}^{2}, x_{1}+2 x_{2}+x_{1} x_{2}^{2}\right)$. Then $F$ is non-degenerate and convenient. We may use Corollary 4.3.11 to conclude that $\mathcal{N}_{\infty}(F)=\emptyset$ and, since $\operatorname{Sing} F=\emptyset$, it follows from Proposition 5.2.1 that $F$ is a diffeomorphism. We remark that $F^{-1}: \mathbb{R}^{2} \rightarrow \mathbb{R}^{2}$ is not a polynomial mapping $\left(F^{-1}\right.$ is a rational mapping).

In the next example, we give a polynomial function $F=\left(f_{1}, f_{2}, f_{3}\right)$ where $\operatorname{Sing} F=$ $\mathcal{N}_{\infty}(F)=\emptyset, F$ is non-degenerate and each $f_{i}$ is non-convenient, $i=1,2,3$. On the other hand, if we consider $G:=\left(f_{1}, f_{3}\right)$ then $G$ is degenerate.

EXAMPLE 5.2.5. Let $F=\left(f_{1}, f_{2}, f_{3}\right): \mathbb{R}^{3} \rightarrow \mathbb{R}^{3}, F(x, y, z)=\left(x+y z+x y^{2}, y, x y+z\right)$. Then $F$ is Newton non-degenerate, non-convenient, $\operatorname{Sing} F=\emptyset$ and $\mathcal{N}_{\infty}(F)=\emptyset$. Thus, our Proposition 5.2.1 yields that $F$ a diffeomorphism. Actually it easy to invert $F$ and see that it is an automorphism. We shall also show that the mapping $G: \mathbb{R}^{3} \rightarrow \mathbb{R}^{2}$ defined by $G=\left(f_{1}, f_{3}\right)$ is degenerate.

The Jacobian matrix of $F$ is given by

$$
\left(\begin{array}{ccc}
1+y^{2} & 2 x y+z & y \\
0 & 1 & 0 \\
y & x & 1
\end{array}\right) .
$$

We can see that the determinant of this matrix is equal to 1 , which implies $\operatorname{Sing} F=\emptyset$.

Now, we will show that $F$ is non-degenerate. From Remark 4.3.4, the set $\left\{F_{\triangle \mathbf{p}} \mid \mathbf{p} \in \mathbb{Z}^{3} \backslash 0\right\}$ has at most 21 elements. These elements are:

Table 5.1: Possibilities for $F_{\triangle_{\mathbf{p}}}$

\begin{tabular}{|l||l||l|}
\hline \hline$F_{\triangle_{1}}=(x, y, z)$ & $F_{\triangle_{8}}=(x+y z, y, z)$ & $F_{\triangle_{15}}=\left(x+y z+x y^{2}, y, z\right)$ \\
\hline$F_{\triangle_{2}}=(x, y, x y)$ & $F_{\triangle_{9}}=\left(x+y z+x y^{2}, y, x y\right)$ & $F_{\triangle_{16}}=\left(x+x y^{2}, y, z\right)$ \\
\hline$F_{\triangle_{3}}=(y z, y, z)$ & $F_{\triangle_{10}}=(y z, y, x y+z)$ & $F_{\triangle_{17}}=\left(x+x y^{2}, y, x y+z\right)$ \\
\hline$F_{\triangle_{4}}=(y z, y, x y)$ & $F_{\triangle_{11}}=\left(x y^{2}, y, x y+z\right)$ & $F_{\triangle_{18}}=(x+y z, y, x y+z)$ \\
\hline$F_{\triangle_{5}}=\left(x y^{2}, y, z\right)$ & $F_{\triangle_{12}}=\left(y z+x y^{2}, y, z\right)$ & $F_{\triangle_{19}}=\left(x+x y^{2}, y, x y\right)$ \\
\hline$F_{\triangle_{6}}=\left(x y^{2}, y, x y\right)$ & $F_{\triangle_{13}}=\left(y z+x y^{2}, y, x y\right)$ & $F_{\triangle_{20}}=\left(y z+x y^{2}, y, x y+z\right)$ \\
\hline$F_{\triangle_{7}}=(x, y, x y+z)$ & $F_{\triangle_{14}}=(x+y z, y, x y)$ & $F_{\triangle_{21}}=\left(x+y z+x y^{2}, y, x y+z\right)$ \\
\hline
\end{tabular}

We have the following situation: 
(a). The cases $F_{\triangle i}$, for $i=1, \ldots, 6$. Since each component of $F_{\triangle i}$ are monomials, the condition $\left(^{*}\right)$ of Definition 4.3.3 is automatically satisfied. This implies the non-degeneracy for these faces.

(b). The cases $F_{\triangle i}$, for $i=7,8$. In these cases, one has $\operatorname{Sing} F_{\triangle i}=\emptyset$, hence these faces are non-degenerated.

(c). In the cases $F_{\triangle i}$, for $i=9, \ldots, 14$, one has $\operatorname{Sing} F_{\triangle i}=\{(x, y, z) \mid y=0\}$. This implies $\operatorname{Sing} F_{\triangle i}=\{(x, y, z) \mid y=0\} \cap\left(\mathbb{R}^{*}\right)^{3}=\emptyset$, which show the non-degeneracy for these faces.

(d). The cases $F_{\triangle_{i}}$, for $i=15, \ldots, 18$. These cases are not possible, i.e., there is no vector $\mathbf{p} \in \mathbb{Z}^{3} \backslash 0$ such that $F_{\triangle_{\mathbf{p}}}=F_{\triangle_{i}}$, for any $i=15, \ldots, 18$. For instance, if we suppose that there exists $\mathbf{p}=\left(p_{1}, p_{2}, p_{3}\right) \in \mathbb{Z}^{3}$ such that $F_{\triangle_{\mathbf{p}}}=F_{\triangle_{15}}$, then the first function of $F_{\triangle_{\mathbf{p}}}$, i.e. $f_{\triangle 1}$, gives the following conditions on $\mathbf{p}: p_{1}=p_{1}+2 p_{2}=p_{2}+p_{3}$, which implies $p_{1}=p_{3}$ and $p_{2}=0$. On the other hand, the function $f_{\triangle \mathbf{p}}$ gives the condition $p_{3}<p_{1}+p_{2}$. Thus, we obtain the following conditions on $\mathbf{p}: p_{1}=p_{3}, p_{2}=0$ and $p_{3}<p_{1}+p_{2}$, which implies the contradictory condition $p_{1}=p_{3}$ and $p_{3}<p_{1}$. Therefore, there is no vector $\mathbf{p} \in \mathbb{Z}^{3}$ such that $F_{\triangle_{\mathbf{p}}}=F_{\triangle_{15}}$. Analogous argument show that the cases $F_{\triangle_{i}}$, for $i=16,17,18$, are not possible.

(e). The cases $F_{\triangle_{19}}, F_{\triangle_{20}}$ and $F_{\triangle_{21}}$. First, we consider the case $F_{\triangle_{19}}$. Let $\mathbf{p}=\left(p_{1}, p_{2}, p_{3}\right) \in$ $\mathbb{Z}^{3}$ such that $F_{\triangle_{19}}=F_{\triangle_{\mathbf{p}}}$. The first function of $F_{\triangle_{\mathbf{p}}}$ gives the following conditions on $\mathbf{p}$ : $p_{1}=p_{1}+2 p_{2}$, which implies $p_{2}=0$. Thus, from definition of $d_{\mathbf{p}}^{j}$ and by the fact that $p_{2}=0$, one has $d_{\mathbf{p}}^{1}=p_{1}, d_{\mathbf{p}}^{2}=0$ and $d_{\mathbf{p}}^{3}=p_{1}$. Since, from definition of non-degenerate, we need only to consider vectors $\mathbf{p} \in \mathbb{Z}^{3}$ such that $\mathrm{N}_{\mathbf{p}} \neq \emptyset$ (see Definition 4.3.3), and since we have shown that $d_{\mathbf{p}}^{2}=0$, which implies $2 \notin \mathrm{N}_{\mathbf{p}}$, we conclude that $1 \in \mathrm{N}_{\mathbf{p}}$ or $3 \in \mathrm{N}_{\mathbf{p}}$. The equations of $f_{\triangle_{\mathbf{p}}^{1}}$ and $f_{\triangle_{\mathbf{p}}^{3}}$ give $\left\{f_{\triangle_{\mathbf{p}}^{1}}=0\right\} \cap\left(\mathbb{R}^{*}\right)^{3}=\emptyset$ and $\left\{f_{\triangle_{\mathbf{p}}^{3}}=0\right\} \cap\left(\mathbb{R}^{*}\right)^{3}=\emptyset$. Therefore, since we have shown that $1 \in \mathrm{N}_{\mathbf{p}}$ or $3 \in \mathrm{N}_{\mathbf{p}}$ and $\left\{f_{\triangle_{\mathbf{p}}}=0\right\} \cap\left(\mathbb{R}^{*}\right)^{3}=\emptyset$ and $\left\{f_{\triangle \triangle_{\mathbf{p}}^{3}}=0\right\} \cap\left(\mathbb{R}^{*}\right)^{3}=\emptyset$, one concludes that the condition $(*)$ of Definition 4.3.3 is satisfied, which implies the non-degeneracy of $F_{\triangle_{19}}$.

Now, we consider the case $F_{\triangle_{20}}$. Let $\mathbf{p} \in \mathbb{Z}^{3}$ such that $F_{\triangle_{20}}=F_{\triangle_{\mathbf{p}}}$. The function $f_{\triangle_{\mathbf{p}}^{1}}$ yields the following condition on $\mathbf{p}: p_{1}+2 p_{2}=p_{2}+p_{3}$ and $p_{1}>p_{1}+2 p_{2}$. The last inequality implies $p_{2}<0$ and consequently one obtains $d_{\mathbf{p}}^{2}<0$. Thus, one has $2 \in \mathrm{N}_{\mathbf{p}}$. Therefore, since $\left\{f_{\triangle_{\mathbf{p}}^{2}}=0\right\} \cap\left(\mathbb{R}^{*}\right)^{3}=\emptyset$ and $2 \in \mathrm{N}_{\mathbf{p}}$, one concludes that the condition $(*)$ of Definition 4.3.3 is satisfied, which shows the non-degeneracy of $F_{\triangle_{20}}$. To finish, we consider the case $F_{\triangle_{21}}$. We have that $F_{\triangle_{21}}=F$ and since we have seen that $\operatorname{Sing} F=\emptyset$, we conclude the non-degeneracy of $F_{\triangle_{21}}$.

Therefore, we have shown the non-degeneracy of $F$. Now, we will show that $G$ is degenerate. Consider the vector $\mathbf{p}:=(-1,-1,-2)$. Then $G_{\triangle_{\mathbf{p}}}=\left(x y^{2}+y z, x y+z\right)$ and we observe that this face corresponds to the face $F_{\triangle_{20}}$ of $F$. We have $\operatorname{Sing} G_{\triangle_{\mathbf{p}}}=\left\{G_{\triangle_{\mathbf{p}}}=\right.$ $0\}=\{(x, y, z) \mid z=-x y\}$ and $\mathrm{N}_{\mathbf{p}}=\{1,2\}$. Thus, if consider $\left(\lambda, \lambda,-\lambda^{2}\right), \lambda \in \mathbb{R}^{*}$, one has $\left(\lambda, \lambda,-\lambda^{2}\right) \in \operatorname{Sing} G_{\triangle_{\mathbf{p}}} \cap\left\{G_{\triangle_{\mathbf{p}}}=0\right\} \cap\left(\mathbb{R}^{*}\right)^{3}$, which show that $G_{\triangle_{\mathbf{p}}}$ is degenerate. 


\subsection{Euler characteristic}

Let $f: \mathbb{R}^{n} \rightarrow \mathbb{R}^{n-1}$ be a smooth mapping. Let $t_{0} \in \mathbb{R}^{n-1}$ be a regular value of $f$. In this subsection we study the topology of $f^{-1}\left(t_{0}\right)$. More precisely, following arguments used in the local case by Szafraniec [51, Theorem 3.1], we present a formula to compute $\chi\left(f^{-1}\left(t_{0}\right)\right)$ (Proposition 5.3.5).

We use in this section some classical definitions, which can be found in Milnor [35, $₫ 4, \S 5$ and §6]. In special, we use the notions of degree of a mapping and index of a vector field (see respectively Milnor [35, page 28 and page 32$]$ ).

We start with the following notation:

Definition 5.3.1. Let $F: \mathbb{R}^{n} \rightarrow \mathbb{R}^{n}$ be a $\mathcal{C}^{1}$ mapping. The Jacobian function of $F$, denoted by $\mathcal{J}_{F}$, is defined as follows $\mathcal{J}_{F}(x):=\operatorname{det}([\operatorname{DF}(x)])$, where $\operatorname{det}(-)$ denotes the determinant function and $[\mathrm{D} F(x)]$ denotes the Jacobian matrix of $F$ at $x$.

We will use in the proof of Proposition 5.3.5 the following three results:

Lemma 5.3.2 (Milnor [35, page 55]). Let $M$ be a smooth connected 1-dimensional manifold without boundary. Then $M$ is diffeomorphic either to the circle $S^{1}$ or to the interval ]0,1[. Moreover, since ] $0,1[$ is diffeomorphic to $\mathbb{R}$, we may assume that $M$ is diffeomorphic either to the circle $S^{1}$ or to $\mathbb{R}$.

Lemma 5.3.3 (Szafraniec [51, Lemma 2.1 (page 79)]). Let $f=\left(f_{1}, \ldots, f_{n-1}\right): \mathbb{R}^{n} \rightarrow \mathbb{R}^{n-1}$ and $h: \mathbb{R}^{n} \rightarrow \mathbb{R}$ be smooth mappings. Let $\mathcal{J}_{(f, h)}$ be the Jacobian function of the map $(f, h)$. We define the mapping $H:=\left(f_{1}, \ldots, f_{n-1}, \mathcal{J}_{(f, h)}\right)$ and consider the Jacobian function of $H$ which one denotes by $\mathcal{J}_{H}$.

Let $t_{0} \in \mathbb{R}^{n-1}$ be a regular value of $f$ and let $x_{0} \in f^{-1}\left(t_{0}\right)$, then:

(a) the restriction $h_{\mid f^{-1}\left(t_{0}\right)}$ has a critical point at $x_{0}$ if and only if $\mathcal{J}_{(f, h)}\left(x_{0}\right)=0$.

(b) the restriction $h_{\mid f^{-1}\left(t_{0}\right)}$ has a non-degenerate critical point at $x_{0}$ if and only if one has $\mathcal{J}_{(f, h)}\left(x_{0}\right)=0$ and $\mathcal{J}_{H}\left(x_{0}\right) \neq 0$.

(c) if $\mathcal{J}_{(f, h)}\left(x_{0}\right)=0$ and $\mathcal{J}_{H}\left(x_{0}\right)>0$ then the restriction $h_{\mid f^{-1}\left(t_{0}\right)}$ has a minimum at $x_{0}$.

(d) if $\mathcal{J}_{(f, h)}\left(x_{0}\right)=0$ and $\mathcal{J}_{H}\left(x_{0}\right)<0$ then the restriction $h_{\mid f^{-1}\left(t_{0}\right)}$ has a maximum at $x_{0}$.

Lemma 5.3.4 (Szafraniec [51, Lemma 2.4 (page 82)]). Let $M$ be a compact 1-dimensional manifold with boundary $\partial M$. Clearly, $\partial M$ is a finite set (see for instance Milnor [35, page 55]). Let $h: M \rightarrow \mathbb{R}$ be a function of class $\mathcal{C}^{2}$. We denote by $\operatorname{Sing}(h)$, the set of critical 
points of $h$. Suppose that $\operatorname{Sing}(h)$ is a finite set subset of $M-\partial M$ and that each critical point of $h$ is non-degenerate. Define $m_{+}:=\#\{x \in \operatorname{Sing}(h) \mid h$ has a minimum at $x\}$ and $m_{-}:=\#\{x \in \operatorname{Sing}(h) \mid h$ has a maximum at $x\}$. Suppose that the following conditions are satisfied:

(a) if $x \in \partial M$ then $h(x) \neq 0$;

(b) if $x \in \partial M$ and $h(x)>0$ then $g$ has a maximum at $x$;

(c) if $x \in \partial M$ and $h(x)<0$ then $g$ has a minimum at $x$.

Then, one has:

$$
\sharp\{x \in \partial M \mid h(x)>0\}-\sharp\{x \in \partial M \mid h(x)<0\}=2\left(m_{+}-m_{-}\right) .
$$

Proposition 5.3.5. Let $f: \mathbb{R}^{n} \rightarrow \mathbb{R}^{n-1}$ be a smooth mapping. Let $t_{0} \in \mathbb{R}^{n-1}$ be a regular value of $f$. Let $\rho: \mathbb{R}^{n} \rightarrow \mathbb{R}$ be the mapping defined by $\rho\left(x_{1}, \ldots, x_{n}\right)=x_{1}^{2}+\ldots+x_{n}^{2}$. We consider the mapping $(f, \rho): \mathbb{R}^{n} \rightarrow \mathbb{R}^{n}$ and the mapping $H: \mathbb{R}^{n} \rightarrow \mathbb{R}^{n}$, defined by $H(x):=$ $\left(f(x)-t_{0}, \mathcal{J}_{(f, \rho)}(x)\right)$, where $\mathcal{J}_{(f, \rho)}$ is the Jacobian function of $(f, \rho)$ (see Definition 5.3.1). Then there exists $R_{0} \in \mathbb{R}$ such that

(i) $\operatorname{deg}\left(\frac{H_{R}}{\left\|H_{R}\right\|}\right)=\frac{1}{2}\left(\sharp\left\{x \in f^{-1}\left(t_{0}\right) \cap S_{R}^{n-1}\right\}\right)$, for any $R \geq R_{0}$.

(ii) $\operatorname{deg}\left(\frac{H_{R}}{\left\|H_{R}\right\|}\right)=\chi\left(f^{-1}\left(t_{0}\right)\right)$, for any $R \geq R_{0}$,

where

$$
\begin{aligned}
\frac{H_{R}}{\left\|H_{R}\right\|}: \quad S_{R}^{n-1}(0) & \rightarrow S_{1}^{n-1}(0) \\
x & \mapsto \frac{H(x)}{\|H(x)\|}
\end{aligned}
$$

for $R \geq R_{0}$.

Proof. We may suppose that all critical points of $\rho_{\mid f^{-1}\left(t_{0}\right)}$ are non-degenerate (otherwise, we take $\tilde{\rho}$ close enough to $\rho$ so that the number in the left size of equations in items (i) and (ii) are the same with $\rho$ and the critical points of $\tilde{\rho}_{\mid f^{-1}\left(t_{0}\right)}$ are non-degenerate). Let $\left\{x_{1}, \ldots, x_{m}\right\} \subset f^{-1}\left(t_{0}\right)$ be the critical points of the restriction $\rho_{\mid f^{-1}\left(t_{0}\right)}$.

By definition of $H$ and from Lemma 5.3.3 item (a), one has $\left\{x_{1}, \ldots, x_{m}\right\}=H^{-1}(0,0)$. From Lemma 5.3.3 item (b), we have $\mathcal{J}_{(f, \rho)}\left(\mathrm{x}_{i}\right)=0$ and $\mathcal{J}_{H}\left(\mathrm{x}_{i}\right) \neq 0$, for $i=1, \ldots, m$. Thus, since $\left\{x_{1}, \ldots, x_{m}\right\}=H^{-1}(0,0)$ and $\mathcal{J}_{H}\left(x_{i}\right) \neq 0$, one concludes that $(0,0) \in \mathbb{R}^{n-1} \times \mathbb{R}$ is a regular value of $H$. 
Let $R_{0} \in \mathbb{R}$ such that $\left\{x_{1}, \ldots, x_{m}\right\} \subset B_{R_{0}}(0)$ and, for any $R \geq R_{0}$, we define the following sets:

$$
\begin{aligned}
& m_{+}:=\left\{x \in H^{-1}(0,0) \cap B_{R} \mid \mathcal{J}_{H}(x)>0\right\}, \\
& m_{-}:=\left\{x \in H^{-1}(0,0) \cap B_{R} \mid \mathcal{J}_{H}(x)<0\right\} .
\end{aligned}
$$

If one considers the mapping

$$
\begin{aligned}
\frac{H_{R}}{\left\|H_{R}\right\|}: \quad S_{R}^{n-1}(0) & \rightarrow S_{1}^{n-1}(0) \\
x & \mapsto \frac{H(x)}{\|H(x)\|},
\end{aligned}
$$

then:

$$
\operatorname{deg}\left(\frac{H_{R}}{\left\|H_{R}\right\|}\right)=m_{+}-m_{-},
$$

where the equality (5.8) follows by definition of index of a mapping (see for instance Milnor [35, §5 and Lemma 4 (page 37)]).

Now, we observe that from Lemma 5.3.3, items (c) and (d), one has the following equalities: $m_{+}=\left\{x \in H^{-1}(0,0) \mid \rho_{\mid f^{-1}\left(t_{0}\right)}\right.$ has a minimum at $\left.x\right\}$ and $m_{-}=\left\{x \in H^{-1}(0,0) \mid\right.$ $\rho_{\mid f^{-1}\left(t_{0}\right)}$ has a maximum at $\left.x\right\}$. We also observe that all conditions of Lemma 5.3.4 are satisfied for $\rho(x)=x_{1}^{2}+\ldots+x_{n}^{2}$, and for the compact 1-dimensional manifold $\overline{B_{R}^{n}(0)} \cap f^{-1}\left(t_{0}\right)$, where $\overline{B_{R}^{n}(0)}$ denotes the topological closure of the $n$-dimensional Euclidean ball of radius $R$. So, from Lemma 5.3.4 and by equality (5.8), one obtains:

$$
2 \operatorname{deg}\left(\frac{H_{R}}{\left\|H_{R}\right\|}\right)=\sharp\left\{x \in f^{-1}\left(t_{0}\right) \cap S_{R}^{n-1} \mid \rho(x)>0\right\}-\sharp\left\{x \in f^{-1}\left(t_{0}\right) \cap S_{R}^{n-1} \mid \rho(x)<0\right\} .
$$

Since $\sharp\left\{x \in f^{-1}\left(t_{0}\right) \cap S_{R}^{n-1} \mid \rho(x)<0\right\}=\emptyset$, we obtain the following equality:

$$
2 \operatorname{deg}\left(\frac{H_{R}}{\left\|H_{R}\right\|}\right)=\sharp\left\{x \in f^{-1}\left(t_{0}\right) \cap S_{R}^{n-1}\right\},
$$

which shows item (i).

To finish, we will show that $\chi\left(f^{-1}\left(t_{0}\right)\right)=\frac{1}{2} \sharp\left\{x \in f^{-1}\left(t_{0}\right) \cap S_{R_{0}}^{n-1}\right\}$, for any $R \geq R_{0}$.

We have supposed that $t_{0}$ is regular value. So, one has that $f^{-1}\left(t_{0}\right)$ is a smooth manifold of dimension one. Let $\left\{\mathcal{C}_{i}\right\}_{i=1}^{l}$ be the connected components of $f^{-1}\left(t_{0}\right)$. From Lemma 5.3.2, we may suppose that there exist $k \leq l$ such that:

$$
\begin{cases}\mathcal{C}_{i} \cong \mathbb{R}, & \text { for } 1 \leq i \leq k, \\ \mathcal{C}_{i} \cong S^{1}, & \text { for } k<i \leq l\end{cases}
$$


From the properties of the Euler characteristic, one has $\chi\left(S^{1}\right)=0, \chi(\mathbb{R})=1$ and $\chi\left(f^{-1}\left(t_{0}\right)\right)=$ $\sum_{i=1}^{l} \chi\left(\mathcal{C}_{i}\right)$. This last equality follows by the fact that $f^{-1}\left(t_{0}\right)$ is disjoint union of $\left\{\mathcal{C}_{i}\right\}$, and by the fact that the Euler characteristic of disjoint union of two manifolds is equal to the sum of their Euler characteristics. Thus, from these properties of Euler characteristic, we conclude that:

$$
\chi\left(f^{-1}\left(t_{0}\right)\right)=\sum_{i=1}^{l} \chi\left(\mathcal{C}_{i}\right)=\sum_{i=1}^{k} \chi\left(\mathcal{C}_{i}\right)=k,
$$

where the second equality follows from $(5.11)\left(\mathcal{C}_{i} \cong S^{1}\right.$, for $\left.k<i \leq l\right)$ and from $\chi\left(S^{1}\right)=0$; the last equality of (5.12) follows again from $(5.11)\left(\mathcal{C}_{i} \cong \mathbb{R}\right.$, for $\left.1 \leq i \leq k\right)$, and from $\chi(\mathbb{R})=1$.

Let us assume that the following condition hold: there exists $\tilde{R}_{0} \in \mathbb{R}$ such that $\sharp\left\{x \in \mathbb{R}^{n} \mid\right.$ $\left.x \in \mathcal{C}_{i} \cap S_{R}^{n-1}\right\}=2$, for any $R \geq \tilde{R}_{0}$ and for $1 \leq i \leq k$ (the proof of which will be given below). If we assume this condition then:

$$
\sum_{i=1}^{k} \sharp\left\{x \in \mathbb{R}^{n} \mid x \in \mathcal{C}_{i} \cap S_{R}^{n-1}\right\}=2 k=2 \chi\left(f^{-1}\left(t_{0}\right)\right), \text { for any } R \geq \tilde{R}_{0},
$$

where the last equality follows by (5.12).

Now, since the connected components $\mathcal{C}_{k+1}, \ldots, \mathcal{C}_{l}$ are compact, there exists $\tilde{\tilde{R}}_{0} \in \mathbb{R}$ such that $\cup_{i=k+1}^{l} \mathcal{C}_{i} \subset B_{\tilde{R}_{0}}(0)$. Therefore, for any $R>\max \left\{R_{0}, \tilde{R}_{0}, \tilde{\tilde{R}}_{0}\right\}$, one has:

$$
2 \operatorname{deg}\left(\frac{H_{R}}{\left\|H_{R}\right\|}\right)=\sharp\left\{x \in f^{-1}\left(t_{0}\right) \cap S_{R}^{n-1}\right\}=\sum_{i=1}^{k} \sharp\left\{x \in \mathcal{C}_{i} \cap S_{R}^{n-1}\right\}=2 \chi\left(f^{-1}\left(t_{0}\right)\right),
$$

where the first equality of (5.14) follows by (5.10); the second equality follows by the fact that $R>\tilde{\tilde{R}}_{0}$, which implies that $f^{-1}\left(t_{0}\right) \cap S_{R}^{n-1}=\left(\cup_{i=1}^{k} \mathcal{C}_{i}\right) \cap S_{R}^{n-1}$; and, finally, the last equality of (5.14) follows by (5.13). Therefore, from (5.14) one obtains item (ii), as desired.

Now, we prove the condition: " $(\star)$ there exists $\tilde{R}_{0} \in \mathbb{R}$ such that $\sharp\left\{x \in \mathbb{R}^{n} \mid x \in\right.$ $\left.\mathcal{C}_{i} \cap S_{R}^{n-1}\right\}=2$, for any $R \geq \tilde{R}_{0}$ and for $1 \leq i \leq k$ ".

Remember that we have assumed $\mathcal{C}_{i} \cong \mathbb{R}$, for $1 \leq i \leq k$. Fix $i$ and take $R_{0} \in \mathbb{R}$ such that $B_{R_{0}}^{n}(0)$ contains the critical values of $\rho_{\mid \mathcal{C}_{i}}$. We will prove the condition $(\star)$ by contradiction.

First, suppose that for some $R \geq R_{0}$, we have $\sharp S_{R}^{n-1} \cap \mathcal{C}_{i}=1$ and let $z$ be the unique element in this intersection. This implies that $C_{i}$ is a disjoint union of the three connected sets $\left(B_{R}^{n}(0) \cap C_{i}\right) \cup\{z\} \cup\left(\left(\mathbb{R}^{n} \backslash \overline{B_{R}^{n}(0)}\right) \cap \mathcal{C}_{i}\right)$. Since $\left(B_{R}^{n}(0) \cap C_{i}\right) \cup\{z\}$ is connected and compact, we obtain that $\mathbb{R} \cong \mathcal{C}_{i}$ can be written as a disjoint union of a connected and compact set $\left(B_{R}^{n}(0) \cap C_{i}\right) \cup\{z\}$ with a open connected set $\left(\left(\mathbb{R}^{n} \backslash \overline{B_{R}^{n}(0)}\right) \cap \mathcal{C}_{i}\right.$. But this is impossible in $\mathbb{R}$ and consequently in $\mathcal{C}_{i}$.

Now, suppose that for some $R \geq R_{0}$, we have that $\sharp S_{R}^{n-1} \cap \mathcal{C}_{i}>2$ and let $z_{1}, z_{2}, z_{3}$ be three elements in this intersection. Since $\mathcal{C}_{i} \cong \mathbb{R}$, we have that $\mathcal{C}_{i} \backslash\left\{z_{1}, z_{2}, z_{3}\right\}$ is a disjoint union of four connected sets $\mathcal{Z}_{1}, \mathcal{Z}_{2}, \mathcal{Z}_{3}, \mathcal{Z}_{4}$ and we may suppose that $\left(\mathcal{Z}_{2} \cup\left\{z_{1}, z_{2}\right\}\right)$ and 
$\left(\mathcal{Z}_{3} \cup\left\{z_{2}, z_{3}\right\}\right)$ are compact sets (where the last condition follows by the fact $\mathbb{R} \cong \mathcal{C}_{i}$ and therefore $\mathcal{C}_{i} \backslash\left\{z_{1}, z_{2}, z_{3}\right\}$ is just $\mathbb{R}$ minus three points). Since we have supposed that $B_{R}^{n}(0)$ contains the critical points of $\rho_{\mid \mathcal{C}_{i}}$, one has that $S_{R}^{n-1}$ is transverse $\mathcal{C}_{i}$ at $z_{2}$, which implies that at least one of the intersections $\mathcal{Z}_{2} \cap\left(\mathbb{R}^{n} \backslash B_{R}^{n}(0)\right), \mathcal{Z}_{3} \cap\left(\mathbb{R}^{n} \backslash B_{R}^{n}(0)\right)$ is not empty. Thus, one may assume that $\mathcal{Z}_{2} \cap\left(\mathbb{R}^{n} \backslash B_{R}^{n}(0)\right)$ is not empty. From this last condition, by definition of $\rho$ and since $\rho\left(z_{1}\right)=\rho\left(z_{2}\right)$, one has that $\rho_{\mid\left(\mathcal{Z}_{2} \cup\left\{z_{1}, z_{2}\right\}\right.}$ has a critical point in $\mathcal{Z}_{2} \cap\left(\mathbb{R}^{n} \backslash B_{R}^{n}(0)\right)$. But this contradicts the fact that $B_{R}^{n}(0)$ contains the critical points of $\rho_{\mid \mathcal{C}_{i}}$.

Therefore, we conclude that for any $R \geq R_{0}$, one has that $\sharp S_{R}^{n-1} \cap \mathcal{C}_{i}=2$, which finishes the proof of the condition $(\star)$ and consequently the proof of proposition. 


\section{Bibliography}

[1] K. Bekka. Regular stratification of subanalytic sets. Bulletin of the London Mathematical Society, 25:7-16, jan 1993. (Cited on pages 36 and 41.)

[2] C. Bivià-Ausina. Injectivity of real polynomial maps and Łojasiewicz exponents at infinity. Math. Z., 257(4):745-767, 2007. (Cited on pages 4, 49, 57, 62, 63 and 71.)

[3] J. Bochnak, M. Coste, and M.-F. Roy. Real algebraic geometry, volume 36 of Ergebnisse der Mathematik und ihrer Grenzgebiete (3) [Results in Mathematics and Related Areas (3)]. Springer-Verlag, Berlin, 1998. Translated from the 1987 French original, Revised by the authors. (Cited on pages 40 and 42.)

[4] S. A. Broughton. On the topology of polynomial hypersurfaces. In Singularities, Part 1 (Arcata, Calif., 1981), volume 40 of Proc. Sympos. Pure Math., pages 167-178. Amer. Math. Soc., Providence, RI, 1983. (Cited on pages 2, 4, 7, 18, 21, 22, 57 and 62.)

[5] S. A. Broughton. Milnor numbers and the topology of polynomial hypersurfaces. Invent. Math., 92(2):217-241, 1988. (Cited on pages 2, 21, 22, 24, 36 and 57.)

[6] Y. Chen. Bifurcation values of mixed polynomials and Newton polyhedra. PhD thesis, 2012. Université Lille 1. (Cited on pages 4, 56 and 57.)

[7] Y. Chen, L. R. G. Dias, and M. Tibăr. On newton non-degeneracy of polynomial mappings. ArXiv e-prints:120\%.1612, 2012. (Cited on pages 4 and 57.)

[8] Y. Chen and M. Tibăr. Bifurcation values of mixed polynomials. Math. Res. Lett., 19(1):59-79, 2012. (Cited on pages 4, 49, 57, 60, 61 and 62.)

[9] D. D'Acunto and V. Grandjean. On gradient at infinity of semialgebraic functions. Ann. Polon. Math., 87:39-49, 2005. (Cited on page 1.)

[10] Z. Denkowska, S. Łojasiewicz, and J. Stasica. Certaines propriétés élémentaires des ensembles sous-analytiques. Bull. Acad. Polon. Sci. Sér. Sci. Math., 27(7-8):529-536 (1980), 1979. (Cited on page 41.)

[11] L. R. G. Dias, M. A. S. Ruas, and M. Tibăr. Regularity at infinity of real mappings and a Morse-Sard theorem. Journal of Topology, 5(2):323-340, 2012. (Cited on pages 17, 39 and 50.)

[12] A. Dimca. Singularities and topology of hypersurfaces. Universitext. Springer-Verlag, New York, 1992. (Cited on page 41.) 
[13] A. H. Durfee. Five definitions of critical point at infinity. In Singularities (Oberwolfach, 1996), volume 162 of Progr. Math., pages 345-360. Birkhäuser, Basel, 1998. (Cited on page 2.)

[14] C. Ehresmann. Les connexions infinitésimales dans un espace fibré différentiable. In Séminaire Bourbaki, Vol. 1, pages Exp. No. 24, 153-168. Soc. Math. France, Paris, 1995. (Cited on page 1.)

[15] G. B. Folland. Real analysis. Pure and Applied Mathematics (New York). John Wiley \& Sons Inc., New York, second edition, 1999. Modern techniques and their applications, A Wiley-Interscience Publication. (Cited on page 9.)

[16] T. Gaffney. Integral closure of modules and Whitney equisingularity. Invent. Math., 107(2):301-322, 1992. (Cited on pages 51, 52 and 55.)

[17] T. Gaffney. Aureoles and integral closure of modules. In Stratifications, singularities and differential equations, II (Marseille, 1990; Honolulu, HI, 1990), volume 55 of Travaux en Cours, pages 55-62. Hermann, Paris, 1997. (Cited on pages 51 and 52.)

[18] T. Gaffney. Fibers of polynomial mappings at infinity and a generalized Malgrange condition. Compositio Math., 119(2):157-167, 1999. (Cited on pages 1, 2, 3, 4, 5, 7, 8, $12,17,19,20,25,26,49,50,52,53,54,55$ and 56.$)$

[19] T. Gaffney, D. Trotman, and L. Wilson. Equisingularity of sections, $\left(t^{r}\right)$ condition, and the integral closure of modules. J. Algebraic Geom., 18(4):651-689, 2009. (Cited on page 51.)

[20] C. G. Gibson, K. Wirthmüller, A. A. du Plessis, and E. J. N. Looijenga. Topological stability of smooth mappings. Lecture Notes in Mathematics, Vol. 552. Springer-Verlag, Berlin, 1976. (Cited on page 41.)

[21] V. Guillemin and A. Pollack. Differential topology. Prentice-Hall Inc., Englewood Cliffs, N.J., 1974. (Cited on page 71.)

[22] H. V. Hà and D. T. Lê. Sur la topologie des polynômes complexes. Acta Math. Vietnam., 9(1):21-32 (1985), 1984. (Cited on pages 1 and 20.)

[23] H. V. Hà and T. S. Phạm. Minimizing polynomial functions. Acta Math. Vietnam., 32(1):71-82, 2007. (Cited on page 1.)

[24] Z. Jelonek. On the generalized critical values of a polynomial mapping. Manuscripta Math., 110(2):145-157, 2003. (Cited on pages 1, 2, 3, 4, 5, 7, 8, 10, 12, 13, 17, 19, 20, 25,26 and 30.) 
[25] Z. Jelonek. On asymptotic critical values and the Rabier theorem. In Geometric singularity theory, volume 65 of Banach Center Publ., pages 125-133. Polish Acad. Sci., Warsaw, 2004. (Cited on pages 5, 7, 8, 10, 15, 16, 17, 19, 20, 69, 70 and 71.)

[26] A. G. Khovanskiı̌. Newton polyhedra, and toroidal varieties. Funkcional. Anal. $i$ Priložen., 11(4):56-64, 96, 1977. (Cited on pages 4, 57 and 67.)

[27] T. C. Kuo. Characterizations of $v$-sufficiency of jets. Topology, 11:115-131, 1972. (Cited on pages $8,10,11$ and 36 .)

[28] K. Kurdyka, T. Mostowski, and A. Parusiński. Proof of the gradient conjecture of R. Thom. Ann. of Math. (2), 152(3):763-792, 2000. (Cited on page 1.)

[29] K. Kurdyka, P. Orro, and S. Simon. Semialgebraic Sard theorem for generalized critical values. J. Differential Geom., 56(1):67-92, 2000. (Cited on pages 1, 2, 3, 5, 8, 10, 11, 13, $17,19,20,25,26,30,39,45$ and 71.$)$

[30] D. T. Lê and C. Weber. Polynômes à fibres rationnelles et conjecture jacobienne à 2 variables. C. R. Acad. Sci. Paris Sér. I Math., 320(5):581-584, 1995. (Cited on page 1.)

[31] M. Lejeune-Jalabert and B. Teissier. Clôture intégrale des idéaux et équisingularité. Ann. Fac. Sci. Toulouse Math. (6), 17(4):781-859, 2008. With an appendix by Jean-Jacques Risler. (Cited on page 52.)

[32] J. N. Mather. Notes on topological stability. Harvard University, Harvard, MA, 1970. (Cited on page 36.)

[33] J. S. Milne. Algebraic geometry (v5.22), 2012. Available at www.jmilne.org/math/. (Cited on page 46.)

[34] J. Milnor. Singular points of complex hypersurfaces. Annals of Mathematics Studies, No. 61. Princeton University Press, Princeton, N.J., 1968. (Cited on pages 22, 24, 33, 36, 37, 52,61 and 65.)

[35] J. Milnor. Topology from the differentiable viewpoint. Princeton Landmarks in Mathematics. Princeton University Press, Princeton, NJ, 1997. Based on notes by David W. Weaver, Revised reprint of the 1965 original. (Cited on pages 74 and 76.)

[36] D. Mumford. Algebraic geometry. I. Classics in Mathematics. Springer-Verlag, Berlin, 1995. Complex projective varieties, Reprint of the 1976 edition. (Cited on page 46.)

[37] D. Mumford. The red book of varieties and schemes, volume 1358 of Lecture Notes in Mathematics. Springer-Verlag, Berlin, expanded edition, 1999. Includes the Michigan lectures (1974) on curves and their Jacobians, With contributions by Enrico Arbarello. (Cited on page 46.) 
[38] A. Némethi. Théorie de Lefschetz pour les variétés algébriques affines. C. R. Acad. Sci. Paris Sér. I Math., 303(12):567-570, 1986. (Cited on pages 2 and 22.)

[39] A. Némethi. Lefschetz theory for complex affine varieties. Rev. Roumaine Math. Pures Appl., 33(3):233-250, 1988. (Cited on pages 2 and 22.)

[40] A. Némethi and A. Zaharia. On the bifurcation set of a polynomial function and Newton boundary. Publ. Res. Inst. Math. Sci., 26(4):681-689, 1990. (Cited on pages 2, 4, 7, 22, 24, 36, 49 and 57.)

[41] R. S. Palais and S. Smale. A generalized Morse theory. Bull. Amer. Math. Soc., 70:165172, 1964. (Cited on page 1.)

[42] A. Parusiński. On the bifurcation set of complex polynomial with isolated singularities at infinity. Compositio Math., 97(3):369-384, jul 1995. (Cited on pages 1, 2, 3, 7, 22, 23, $24,29,47$ and 55.)

[43] A. Parusiński. A note on singularities at infinity of complex polynomials. In Symplectic singularities and geometry of gauge fields (Warsaw, 1995), volume 39 of Banach Center Publ., pages 131-141. Polish Acad. Sci., Warsaw, 1997. (Cited on pages 20, 22, 23, 24 and 34.)

[44] A. Parusiński. Topological triviality of $\mu$-constant deformations of type $f(x)+t g(x)$. Bulletin of the London Mathematical Society, 31:686-692, nov 1999. (Cited on pages 1 and 23.)

[45] L. Păunescu and A. Zaharia. On the łojasiewicz exponent at infinity for polynomial functions. Kodai Math. J., 20(3):269-274, 1997. (Cited on pages 1, 3, 23, 36 and 38.)

[46] F. Pham. Vanishing homologies and the $n$ variable saddlepoint method. In Singularities, Part 2 (Arcata, Calif., 1981), volume 40 of Proc. Sympos. Pure Math., pages 319-333. Amer. Math. Soc., Providence, RI, 1983. (Cited on page 2.)

[47] F. Pham. La descente des cols par les onglets de Lefschetz, avec vues sur Gauss-Manin. Asterisque, (130):11-47, 1985. (Cited on pages 2, 22 and 57.)

[48] P. J. Rabier. Ehresmann fibrations and palais-smale conditions for morphisms of finsler manifolds. Annals of Mathematics, 146(3):647-691, nov 1997. (Cited on pages 1, 2, 3, 5, $7,8,10,11,17,19,20,25,26$ and 39.)

[49] D. Siersma and M. Tibăr. Singularities at infinity and their vanishing cycles. Duke Mathematical Journal, 80(3):771-783, dec 1995. (Cited on pages 1, 2, 3, 7, 20, 22, 23, 24, 29, 34 and 47.) 
[50] M. Suzuki. Propriétés topologiques des polynômes de deux variables complexes, et automorphismes algébriques de l'espace $\mathbf{C}^{2}$. J. Math. Soc. Japan, 26:241-257, 1974. (Cited on pages 1 and 20.)

[51] Z. Szafraniec. On the number of branches of a 1-dimensional semianalytic set. Kodai Math. J., 11(1):78-85, 1988. (Cited on page 74.)

[52] B. Teissier. Cycles évanescents, sections planes et conditions de Whitney. In Singularités à Cargèse (Rencontre Singularités Géom. Anal., Inst. Études Sci., Cargèse, 1972), pages 285-362. Astérisque, Nos. 7 et 8. Soc. Math. France, Paris, 1973. (Cited on page 52.)

[53] M. Tibăr. Asymptotic equisingularity and topology of complex hypersurfaces. Internat. Math. Res. Notices, (18):979-990, 1998. (Cited on pages 2, 23, 24, 29 and 50.)

[54] M. Tibăr. Topology at infinity of polynomial mappings and Thom regularity condition. Compositio Math., 111(1):89-109, 1998. (Cited on pages 1, 3, 23, 29, 41 and 42.)

[55] M. Tibăr. Regularity at infinity of real and complex polynomial functions. In Singularity theory (Liverpool, 1996), volume 263 of London Math. Soc. Lecture Note Ser., pages 249-264. Cambridge Univ. Press, Cambridge, 1999. (Cited on pages 1, 2, 3, 23, 24, 29 and 36.)

[56] M. Tibăr. Polynomials and vanishing cycles, volume 170 of Cambridge Tracts in Mathematics. Cambridge University Press, Cambridge, 2007. (Cited on pages 1, 2, 7, 22, 23, 24, 29 and 42.)

[57] M. Tibăr and A. Zaharia. Asymptotic behaviour of families of real curves. Manuscripta Math., 99(3):383-393, 1999. (Cited on pages 1, 21, 47 and 48.)

[58] A. van den Essen. Polynomial automorphisms and the Jacobian conjecture, volume 190 of Progress in Mathematics. Birkhäuser Verlag, Basel, 2000. (Cited on page 71.)

[59] H. Whitney. Local properties of analytic varieties. In Differential and Combinatorial Topology (A Symposium in Honor of Marston Morse), pages 205-244. Princeton Univ. Press, Princeton, N. J., 1965. (Cited on pages 41 and 46.)

[60] N. Young. An introduction to Hilbert space. Cambridge Mathematical Textbooks. Cambridge University Press, Cambridge, 1988. (Cited on page 9.) 